\title{
On properties of relatively hyperbolic groups
}

by

Ming Ming Zhang

A thesis submitted to the Faculty of Graduate and Postdoctoral Affairs in partial fulfillment of the requirements for the degree of

Doctor of Philosophy

in

Pure Mathematics

Carleton University

Ottawa, Ontario

(C) 2017

Ming Ming Zhang 


\section{Abstract}

We discuss a number of problems in relatively hyperbolic groups. We show that the word problem and the conjugacy (search) problem are solvable in linear and quadratic time, respectively, for a relatively hyperbolic group, whenever the corresponding problem is solvable in linear and quadratic time in each parabolic subgroup. We also consider the class $\mathscr{R}$ of finitely generated toral relatively hyperbolic groups. We show that groups from $\mathscr{R}$ are commutative transitive and generalize a theorem proved by Baumslag in [Bau67] to $\mathscr{R}$. Moreover, we discuss two definitions of (fully) residually- $\mathscr{C}$ groups, the classical Definition 1.2.1 and a modified Definition 1.2.5. Building upon results obtained by Ol'shanskii [Ol'93] and Osin Osi10], we prove the equivalence of the two definitions for $\mathscr{C}=\mathscr{R}$. This is a generalization of the similar result obtained by Ol'shanskii for the class of torsion-free hyperbolic groups. Let $\Gamma \in \mathscr{R}$ be non-abelian and non-elementary. Kharlampovich and Miasnikov proved in [KM12] that a finitely generated fully residually- $\Gamma$ group $G$ embeds into an iterated extension of centralizers of $\Gamma$. We deduce from their theorem that every finitely generated fully residually- $\Gamma$ group embeds into a group from $\mathscr{R}$. On the other hand, we give an example of a finitely generated torsion-free fully residually- $\mathscr{H}$ group that does not embed into a group from $\mathscr{R} ; \mathscr{H}$ is the class of hyperbolic groups.

\section{Acknowledgements}

The individual who deserves my foremost gratitude is my advisor, Inna Bumagin. I am grateful to her guidance, motivation and encouragement. I would also like to thank to my family and friends for their support. Finally, I would like to express my deep gratitude to the anonymous bone marrow donor who helped to save my life. 


\section{Contents}

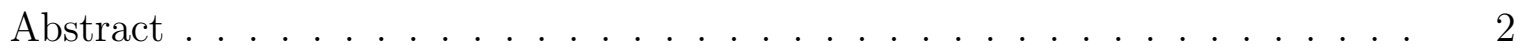

Acknowledgements . . . . . . . . . . . . . . . . . . 2

\begin{tabular}{lll}
\hline & Introduction & 4
\end{tabular}

1.1 Algorithmic problems . . . . . . . . . . . . . . . . . . . . 5

1.2 Residual property . . . . . . . . . . . . . . . . . . . . . . . . . . . . . . 9

1.3 Outline of the thesis . . . . . . . . . . . . . . . . . . . 11

$\begin{array}{llr}2 & \text { Preliminaries } & 12\end{array}$

2.1 Cayley graphs . . . . . . . . . . . . . . . . . . . . . . . . . 12

2.2 Hyperbolic groups . . . . . . . . . . . . . . . . . . . . . . . . . . . . . . 14

2.3 Relatively hyperbolic groups . . . . . . . . . . . . . . . . . . . . . . 16

2.3 .1 The definition of $\mathrm{O} \sin$. . . . . . . . . . . . . . 16

2.3 .2 The definition of Farb . . . . . . . . . . . . . . . . 17

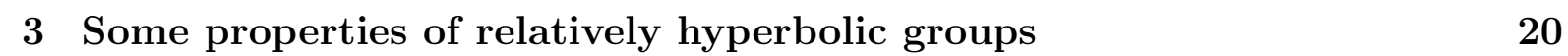

3.1 Equivalent definitions of relative hyperbolicity . . . . . . . . . . . . . . 20

3.2 Some properties . . . . . . . . . . . . . . . . . . . . . . 21

3.3 Small cancellations . . . . . . . . . . . . . . . . . . . . . . . . . . 24

3.4 Combination theorems . . . . . . . . . . . . . . 26

$\begin{array}{|ll|}4 & \text { Some algorithmic problems on relatively hyperbolic groups } \\ 28\end{array}$

4.1 The word problem . . . . . . . . . . . . . . . . 28

4.1 .1 Estimates . . . . . . . . . . . . . . . . . . . . . . . . 28

4.1 .2 The algorithm . . . . . . . . . . . . . . . . . . . 32

$4.1 .3 \quad$ The time complexity of the algorithm . . . . . . . . . . . . 35

4.2 The conjugacy problem $\ldots \ldots \ldots \ldots$. . . . . . . . . . . . . . . . . 39

4.2 .1 Estimates . . . . . . . . . . . . . . . . . . . . . . . 39

4.2 .2 The algorithm and complexity . . . . . . . . . . . . . . . . . . 42

$\begin{array}{|ll|}5 & \text { Residual property of relatively hyperbolic groups }\end{array}$

5.1 Residual homomorphisms . . . . . . . . . . . . . . . . . 45

5.2 The generalization of Baumslag's theorem . . . . . . . . . . . . . . 47

5.3 Toral-limit groups . . . . . . . . . . . . . . . . . . . . . . . . 48

5.4 The Baumslag-Solitar groups . . . . . . . . . . . . . . . . 50

$\begin{array}{lll}6 & \text { Future directions } & 52\end{array}$

6.1 The conjugacy problem for relatively hyperbolic groups . . . . . . . . . . 52

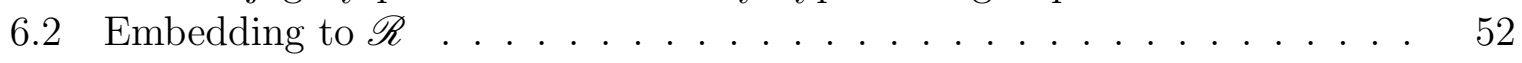




\section{Chapter 1}

\section{Introduction}

This thesis focuses on one of the mathematical areas: geometric group theory, that is the study of finitely generated groups as geometric objects. The simplest way to treat a group as a geometric object is via its Cayley graph. Briefly, given a finite generating set $S$ of a group $G$, the Cayley graph $\Gamma(G, S)$ of $G$ with respect to $S$ is a graph whose vertices are the elements of $G$, and whose edges are the generators $s$ from $S$. An edge labeled by $s$ originates at $x$ and terminates at $y$ whenever $x s=y$; see Section 2.1 for details. Using techniques from topology, geometric properties of the Cayley graph of $G$ can disclose concealed combinatorial and algebraic properties of $G$. Thus, geometric group theory lies at the juncture of algebra and topology; in particular, it closely interacts with low-dimensional topology, hyperbolic geometry, differential geometry, combinatorial group theory, complexity theory, mathematical logic, and other areas of mathematics.

In 1987, the notion of a hyperbolic group, also known as a word hyperbolic group, was introduced and developed by Gromov in Gro87. A finitely generated group $G$ is said to be hyperbolic if for any finite generating set $S$, the Cayley graph $\Gamma(G, S)$ has the property that there is a positive real constant $\delta$ such that all geodesic triangles are $\delta$-thin. This means that for any geodesic triangle in $\Gamma(G, S)$, the closed $\delta$-neighborhood of any two sides contains the third side; see Section 2.2 for details. The theory of hyperbolic groups covers a vast category of groups, for example, all finite groups and finitely generated free groups; see Examples 2.2.3 for details. Moreover, other examples include the fundamental groups of compact Riemannian manifolds with negative sectional curvature and certain small cancellation groups; see the book [GdLH, Chapter 1] for details. An elaboration of the ideas of [Gro87] can be found, for instance, in the book [CDP89]. Notably, Ol'shanskii in Ol'92] showed that "almost" every finitely presented group is hyperbolic in a definite statistical meaning. Roughly, the main idea starts with all presentations of groups with $m$ generators and $n$ relators. Given positive numbers $d_{1}, \cdots, d_{n}$ with $d_{1}+\cdots+d_{n}=d$, we define by $N_{d}=N_{d}(m, n)$ the number of all presentations of groups which have relators of length $d_{1}, \cdots, d_{n}$, respectively, and $N_{d}^{h}=N_{d}^{h}(m, n)$ the number of these which are hyperbolic. Then $\lim _{d \rightarrow \infty} \frac{N_{d}^{h}}{N_{d}}=1$; this means that a group taken at random is probably hyperbolic.

In order to understand the behaviour of "almost" every group, perhaps one should consider the study of hyperbolic groups. However, the class of hyperbolic groups does not contain all finitely generated groups. For instance, the free rank 2 abelian group $\mathbb{Z}^{2}$, which is also a certain small cancellation group, is not hyperbolic. Indeed, its Cayley graph presented in the Euclidean plane is not hyperbolic. More generally, no hyperbolic group has a subgroup isomorphic to $\mathbb{Z}^{2}$. This is due to the fact that the centralizer 
$C(g)$ of an infinite order element $g$ of a hyperbolic group is such that the cyclic group generated by $g$ has finite index in $C(g)$; see [BH99, Corollary 3.10, Chapter III.Г]. Now, an interesting case can happen when a group $G$ which itself is not hyperbolic, contains $\mathbb{Z}^{2}$ as a subgroup, and after contracting the Cayley graph of $G$ along all cosets of $\mathbb{Z}^{2}$, the resulting graph is hyperbolic. In this case, the group $G$ combines a nontrivial mix of hyperbolicity and nilpotency. This motivating example expands the class of hyperbolic groups. With a technical condition, we say that the group $G$ is hyperbolic relative to the subgroup $\mathbb{Z}^{2}$. In general, a group $\Gamma$ can be hyperbolic relative to a preferred class of subgroups $\left\{H_{1}, \cdots, H_{m}\right\}$, called parabolic subgroups. Such a larger class of groups $\Gamma$ is called relatively hyperbolic groups. An element $g$ of $\Gamma$ is called parabolic if it lies in a conjugate of some parabolic subgroup $H_{i}$; otherwise, the element $g$ is called hyperbolic.

In the same paper [Gro87], Gromov introduced the notion of a relatively hyperbolic group as a generalization of a hyperbolic group. Later, it was reformulated and studied from different points of view by Farb [Far98, Bowditch Bow12, Osin Osi06c and many others. The class of relatively hyperbolic groups includes many examples. For instance, any hyperbolic group is hyperbolic relative to the trivial subgroup; and one of the motivating examples [Far98], if $M$ is a complete finite-volume manifold of pinched negative sectional curvature, then the fundamental group $\pi_{1}(M)$ is hyperbolic relative to the cusp subgroups, where the cusp subgroups are nilpotent. Geometrically finite Kleinian groups are relatively hyperbolic groups [Bow12. Other examples include free products of finitely many finitely generated groups as they are hyperbolic relative to the factors, small cancellation quotients of free products [Osi06c, and finitely generated fully residually free groups [KM96].

The definitions of Gromov, Bowditch and Osin allow infinitely generated relatively hyperbolic groups; such generalization is discussed by Hruska in [Hru10]. In contrast, infinitely generated groups are not considered in the theory of hyperbolic groups. Such a generalization, a picture of the global structure of groups and a mix of the properties of groups from different classes lead us to the study of relatively hyperbolic groups. It is worth noting that the notion of relative hyperbolicity has been generalized by Dahmani, Guirardel and Osin in [DGO17]. Also, relatively hyperbolic groups are, in particular, acylindrically hyperbolic, as it was proved by Osin in [Osi16].

In this thesis, we will investigate certain algorithmic and algebraic properties of relatively hyperbolic groups, in particular, the algorithmic problems and the residual property.

\section{$1.1 \quad$ Algorithmic problems}

Three fundamental algorithmic problems in group theory were proposed by Max Dehn in 1911 [Deh11], namely, the word problem, the conjugacy problem and the isomorphism problem. The word and conjugacy problems for a finitely presented group $G$ will be considered in this thesis and they are identified as follows.

- The Word Problem. One asks to find an algorithm that decides whether or not an arbitrary element of $G$ given as a product of generators is the identity.

- The Conjugacy Problem. One is required to find an algorithm that decides, given any two elements $x$ and $y$ of $G$, whether $x$ and $y$ are conjugate, i.e., whether there is a conjugating element $z$ of $G$ such that $x=z y z^{-1}$. 
When we say an algorithmic problem is solvable, we mean that there is an algorithm that solves the problem; otherwise, the algorithmic problem is called unsolvable. The conjugacy problem is known to be unsolvable for finitely presented groups in general, which was proven by Novikov Nov54 in 1954. Three years later, a different proof was obtained independently by Boone [Boo59]. In 1955, Novikov proved that the word problem is unsolvable in [Nov55]. We note that the word problem is solvable if the conjugacy problem is solvable. Indeed, the algorithm solving the conjugacy problem decides whether the given element is conjugate to the identity. The converse is not necessarily true as Fridman [Fri60] showed that there exists a finitely presented group with the solvable word problem and unsolvable conjugacy problem. However, for the class of hyperbolic groups, Gromov stated in [Gro87] that the word and conjugacy problems are solvable.

According to [Gro87], Dehn's algorithm can be employed as a solution to the word problem for hyperbolic groups. In [Deh12, Dehn provided an algorithm that solves the word problem for the fundamental groups of closed orientable two-dimensional manifolds of genus greater than or equal to 2. By now, such algorithm for solving the word problem of a group is called Dehn's algorithm and it can be described as follows.

- Dehn's alogrithm. Let $\langle X \mid \mathcal{R}\rangle$ be a finite presentation of a group $G$, where $X$ is a finite generating set and $\mathcal{R}$ is a finite set of relators; and let $w$ be a non-empty word in the alphabet $X^{ \pm 1}$ (i.e., $w$ represents an element of $G$ as a product of generators in $X^{ \pm 1}$ ). One looks for a subword $u$ of $w$ that is a subword of a relator $R=u v \in \mathcal{R}$ such that $\|u\|>\|v\|$; here $\|z\|$ denotes the length of a word $z$. If there is no such subword, one terminates and outputs that $w$ does not represent the identity; otherwise, one replaces $u$ with $v^{-1}$ and repeats the search for a subword of the edited word $w^{\prime}$, where $w^{\prime}$ represents the same element as $w$ such that $\left\|w^{\prime}\right\|<\|w\|$. After at most $\|w\|$ steps, one will have either reduced to the empty word or verified that $w$ does not represent the identity.

In solving the conjugacy problem for a hyperbolic group $H$, the key idea is as follows. If the given elements $x$ and $y$ are conjugate in $H$ then the length of a shortest conjugating element $z$ is linearly bounded in terms of the sum of the lengths of $x$ and $y$; this was proven by Lysenok in Lys90. For each $z$, Dehn's algorithm is applied to the element $z y z^{-1} x^{-1}$. If $z y z^{-1} x^{-1}$ is the identity for some $z$, one stops and declares that $x$ and $y$ are conjugate, and then, outputs the conjugating element $z$; otherwise, one declares that $x$ and $y$ are not conjugate. Such an algorithm is closely related to the conjugacy search problem which can be formulated as follows.

- The Conjugacy Search Problem. Given any two elements $x$ and $y$ of a group $G$ and the information that $x$ and $y$ are conjugate in $G$, one is required to find a conjugating element.

It is known that the conjugacy problem is solvable for many other classes of groups, for example, mapping class groups [MM00], free solvable groups [Vas11, nilpotent groups [MMNV15, and biautomatic groups GS91b. It follows that these classes of groups also have solvable word problem.

A significant part of this thesis deals with efficient algorithms which are measured by the time complexity. That is, the amount of time taken by an algorithm to run expressed using big $\mathrm{O}$ notation. Let $n$ be the size of the input. As Dehn's algorithm terminates after at most $n$ steps, the time complexity of Dehn's algorithm solving the word problem 
for hyperbolic groups is $\mathcal{O}(n)$. Let $u$ and $v$ be elements in a hyperbolic group $H$. We denote by $[u]_{H}$ and $[v]_{H}$ the conjugacy classes of $u$ and $v$ in $H$, respectively. In solving the conjugacy (search) problem for $H$, a constant bound of the length of a conjugating element $w$ for some representatives of $[u]_{H}$ and $[v]_{H}$ was found by Bridson and Haefliger in [BH99, Chapter III.Г]. For each conjugating element $w$ and certain representatives $u^{\prime}$ of $[u]_{H}$ and $v^{\prime}$ of $[v]_{H}$, Dehn's algorithm is applied to the element $w v^{\prime} w^{-1} u^{\prime-1}$. Since the number of $w$ 's is a constant which is independent of $u$ and $v$, this leads to a cubic time $\mathcal{O}\left(n^{3}\right)$ algorithm. Later, the result of cubic time was improved by Bridson and Howie in [BH05] to quadratic time $\mathcal{O}\left(n^{2}\right)$. Their idea allows one to avoid applying Dehn's algorithm on every $v^{\prime}$; instead, one applies Dehn's algorithm to the element $w v w^{-1} u^{\prime-1}$ for each $w$ and every $u^{\prime}$. Epstein and Holt in [EH06] were able to improve further to quasilinear time $\mathcal{O}(n \log n)$. In summary, there exist efficient algorithms that solve the word and conjugacy (search) problems in hyperbolic groups. As a generalization of hyperbolic groups, a natural question for finitely generated relatively hyperbolic groups can be asked as follows.

Question 1.1.1. Are there "efficient" algorithms solving the word and conjugacy problems in relatively hyperbolic groups, whenever the corresponding problems are solvable in parabolic subgroups?

Farb in [Far98] generalized Dehn's algorithm in hyperbolic groups to relatively hyperbolic groups.

Theorem 1.1.2. [Far98, Theorem 3.7] Suppose that $G$ is a finitely generated group hyperbolic relative to the set of subgroups $\left\{H_{1}, \cdots, H_{m}\right\}$ and that $H_{i}$ has word problem solvable in time $\mathcal{O}(f(n))$ for all $1 \leq i \leq m$. Then there is an algorithm that gives an $\mathcal{O}(f(n) \log n)$-time solution to the word problem for $G$.

Farb's algorithm applies the solution of the word problem in each parabolic subgroup every time when it appears. On the other hand, our approach allows one to avoid some unnecessary computations in the parabolic subgroups by checking a condition. This leads us to an $\mathcal{O}(f(n))$-time algorithm. In Section 4.1 we prove the following theorem.

Theorem 1.1.3. Suppose that $G$ is a finitely generated group hyperbolic relative to the set of subgroups $\left\{H_{1}, \cdots, H_{m}\right\}$ and that $H_{i}$ has word problem solvable in time $\mathcal{O}(f(n))$ for all $1 \leq i \leq m$ where $f(n) \succeq \mathcal{O}(n)$. Then there is an algorithm that gives an $\mathcal{O}(f(n))$-time solution to the word problem for $G$.

Solvability of the conjugacy problem in relatively hyperbolic groups was claimed by Gromov in Gro87, and an algorithm was given by Bumagin in Bum04 as well as Osin in Osi06c for hyperbolic elements. Both algorithms are considered as inefficient. A polynomial bound of the shortest length of a conjugating element was found by Ji, Olge and Ramsey in [JOR10]. An improved polynomial bound was given by O'Connor in O'C12]. In [Bum15], Bumagin provided an "efficient" algorithm, as follows.

Theorem 1.1.4. Bum15, Theorem 5.8] Suppose that $G$ is a finitely generated group hyperbolic relative to the set of subgroups $\left\{H_{1}, \cdots, H_{m}\right\}$ and that $H_{i}$ has word and conjugacy problem solvable in time $\mathcal{O}(f(n))$ and $\mathcal{O}(g(n))$, respectively, for all $1 \leq i \leq m$. Then there is an algorithm that decides for each one of $u$ and $v$ in $G$ whether the element is hyperbolic or parabolic, and then does the following. 
1. If one of $u$ and $v$ is hyperbolic and the other one is parabolic in $G$ then the algorithm stops and declares that $u$ and $v$ are not conjugate. The time complexity of the procedure in this case is $\mathcal{O}(n f(n))$.

2. If both $u$ and $v$ are hyperbolic, then the algorithm provides an $\mathcal{O}\left(n^{2} f(n) \log n\right)$-time solution to the conjugacy (search) problem for $G$.

3. If both $u$ and $v$ are parabolic, then the algorithm gives an $\max \{\mathcal{O}(g(n)), \mathcal{O}(n f(n))\}$ time solution to the conjugacy problem for $G$.

We are able to further refine the time complexity of Bumagin's algorithm in Theorem 1.1.4 by using Theorem 1.1.3 and Bridson and Howie's idea for hyperbolic groups. In section 4.2 we prove the following theorem.

Theorem 1.1.5. Suppose that $G$ is a finitely generated group hyperbolic relative to the set of subgroups $\left\{H_{1}, \cdots, H_{m}\right\}$ and that $H_{i}$ has word and conjugacy problem solvable in time $\mathcal{O}(f(n))$ and $\mathcal{O}(g(n))$, respectively, for all $1 \leq i \leq m$. Then there is an algorithm that gives a $\max \{\mathcal{O}(n f(n)), \mathcal{O}(g(n))\}$-time solution to the conjugacy problem for $G$.

As an immediate corollary of Theorem 1.1.3 and 1.1.5, we have the following estimates for the time complexity of the word and conjugacy problems.

Corollary 1.1.6. With the assumptions and notation in Theorem 1.1.5, we have the following:

1. Suppose that $H_{i}$ has conjugacy search problem solvable in time $\mathcal{O}(h(n))$ for all $1 \leq$ $i \leq m$. Then there is an algorithm that gives an $\max \{\mathcal{O}(n f(n)), \mathcal{O}(g(n)), \mathcal{O}(h(n))\}$ time solution to the conjugacy search problem for $G$.

2. If each $H_{i} \in \mathcal{H}$ of $G$ is abelian, then the word and conjugacy problems are solvable in time $\mathcal{O}(n)$ and $\mathcal{O}\left(n^{2}\right)$.

3. If each $H_{i} \in \mathcal{H}$ of $G$ is free solvable, then the word and conjugacy problems are solvable in time $\mathcal{O}\left(n^{3}\right)$ and $\mathcal{O}\left(n^{8}\right)$, respectively.

4. If each $H_{i} \in \mathcal{H}$ of $G$ is nilpotent, then the word and conjugacy problems are solvable in time $\mathcal{O}(n \log n)$ and $\mathcal{O}\left(n^{2} \log n\right)$, respectively.

Proof. Theorem 1.1.4 and 1.1.5 imply the first assertion. Note that if each $H_{i}$ is abelian then $\mathcal{O}(f(n))=\mathcal{O}(g(n))=\mathcal{O}(n)$. The second assertion immediately follows from Theorems 1.1.3 and 1.1.5. The third assertion is due to [MRUV10] and [Vas11], the word and conjugacy problems in free solvable groups are solvable in time $\mathcal{O}\left(n^{3}\right)$ and $\mathcal{O}\left(n^{8}\right)$, respectively. The last assertion is due to [MMNV15], the word and conjugacy problems in nilpotent groups are solvable in quasi-linear and quasi-quadratic time, respectively.

Our results have implications for group-based cryptography. In the last few years, there have been considerable interest in selecting a suitable group in a protocol based on the difficulty of the conjugacy search problem; see, for instance, [MSU11, Chapter 4]. Roughly, one would like a group $\Gamma$ satisfying the following conditions:

(P1) there is an efficient (linear or quadratic time) algorithm solving the word problem for $\Gamma$; 
(P2) there does not exist a sub-exponential time algorithm solving the conjugacy search problem for $\Gamma$.

Theorem 1.1.3 and the first statement of Corollary 1.1.6 show that one should give up considering the class of relatively hyperbolic groups as a good candidate. Indeed, if $G$ is a finitely generated group hyperbolic relative to the set of subgroups $\left\{H_{1}, \cdots, H_{m}\right\}$, and if $G$ is a good choice for the protocol, then each $H_{i}$ satisfies both (P1) and (P2).

\section{$1.2 \quad$ Residual property}

The second part of this thesis will focus on the residual property of relatively hyperbolic groups. It is based on the work of [BZ16].

The notion of a (fully) residually- $\mathcal{C}$ group, where $\mathcal{C}$ is a class of groups, was introduced long time ago. Usually, $\mathcal{C}$ is chosen to be a class of groups with nice properties, such as the class of all finite groups, nilpotent groups, free groups, etc. The classical definitions are as follows.

Definition 1.2.1 (Classical Definition). Let $\mathcal{C}$ be a class of groups. A group $G$ is called a residually-C group if for every nontrivial element $1 \neq g \in G$ there is a group $H_{g} \in \mathcal{C}$ and an onto homomorphism $\phi_{g}: G \rightarrow H_{g}$, such that $\phi_{g}(g) \neq 1$. A group $G$ is called a fully residually-C group if for every finite set $S=\left\{g_{1}, \ldots, g_{n}\right\}$ of distinct elements of $G$ there is a group $H_{S} \in \mathcal{C}$ and an onto homomorphism $\phi_{S}: G \rightarrow H_{S}$, such that the images $\phi_{S}\left(g_{1}\right), \ldots, \phi_{S}\left(g_{n}\right)$ are all distinct in $H_{S}$.

Clearly, every fully residually- $\mathcal{C}$ group is, in particular, residually- $\mathcal{C}$. For some classes $\mathcal{C}$, the opposite also holds. It is not hard to show that every residually finite group is fully residually finite and that every residually nilpotent group is fully residually nilpotent, as follows. Let $S=\left\{g_{1}, \cdots, g_{n}\right\}$ be a finite set of distinct elements of a group $G$. Then $g_{i} g_{j}^{-1} \neq 1$ for any $1 \leq i \neq j \leq n$. If $G$ is a residually finite (respectively, nilpotent) group, then there is a finite (respectively, nilpotent) group $H_{i, j}$ and an onto homomorphism $\phi_{i, j}: G \rightarrow H_{i, j}$ such that $\phi_{i, j}\left(g_{i} g_{j}^{-1}\right) \neq 1$; and so, $\phi_{i, j}\left(g_{i}\right) \neq \phi_{i, j}\left(g_{j}\right)$; hence, for all $1 \leq i \neq j \leq n$, the direct product of $H_{i, j}$ 's, denoted by $H$ is a finite (respectively, nilpotent) group and $\phi: G \rightarrow H$ such that $\phi\left(g_{i} g_{j}\right)=\phi_{i, j}\left(g_{i} g_{j}\right)$ is an onto homomorphism. It follows that $G$ is fully residually finite (respectively, nilpotent). The situation is very different for the class of free groups, as was shown by Baumslag in [Bau67.

Theorem 1.2.2 (B. Baumslag [Bau67]). A group is fully residually free if and only if it is residually free and commutative transitive.

Recall that a group $G$ is called commutative transitive if for any nontrivial elements $g, h, f \in G$, if $[g, h]=1$ and $[g, f]=1$ then $[h, f]=1$. There are residually free groups which are not commutative transitive, for example, the direct product of free groups of rank $2, F_{2} \times F_{2}$. Indeed, $F_{2} \times F_{2}$ is residually free by taking a projection homomorphism onto one of components $F_{2}$; and $F_{2} \times F_{2}=\langle a, b \mid-\rangle \times\langle x, y \mid-\rangle$ is not commutative transitive because $[a, x]=1=[b, x]$ while $[a, b]$ is nontrivial in the free group $\langle a, b \mid-\rangle$. We generalize Baumslag's theorem to the wider class $\mathscr{R}$.

Definition 1.2.3. The set of $\mathscr{R}$ is the class of finitely generated toral relatively hyperbolic groups, that is, finitely generated torsion-free groups hyperbolic relative to a collection of abelian subgroups. 
In Section 5.2 we prove the following theorem.

Theorem 1.2.4. A finitely generated group is fully residually- $\mathscr{R}$ if and only if it is residually- $\mathscr{R}$ and commutative transitive.

A generalization of Theorem 1.2.2 to some classes of groups was obtained by Ciobanu, Fine and Rosenberger in [CFR16]. Whereas some of the groups they consider are in the class $\mathscr{R}$, other groups have torsion and thus are not covered by our Theorem 1.2.4.

Our motivation is rooted in the growing interest to the algebraic geometry over toral relatively hyperbolic groups. Fully residually free groups play a critical role in the theory of equations over free groups and therefore, in the solution to the famous Tarski's problems [Sel06], [KM06] and [KM98a], [KM98b], [BMR99], where the basics of the algebraic geometry over free groups are developed. Later, the solution to the Tarski problem was generalized to torsion-free hyperbolic groups; see [Sel09], KM13] and [GW16]. Sela introduced limit groups and showed that limit groups are precisely finitely generated fully residually free groups [Sel01].

However, one changes the point of view slightly when studying algebraic geometry over (relatively) hyperbolic groups. Firstly, one has to consider all homomorphisms, not only epimorphisms; in other words, one uses the following Definition 1.2.5, rather than Definition 1.2.1.

Definition 1.2.5 (Working Definition). Let $\mathcal{C}$ be a class of groups. A group $G$ is called a residually-C group if for every nontrivial element $1 \neq g \in G$ there is a group $H_{g} \in \mathcal{C}$ and a homomorphism $\phi_{g}: G \rightarrow H_{g}$, such that $\phi_{g}(g) \neq 1$. A group $G$ is called a fully residually-C group if for every finite set $S=\left\{g_{1}, \ldots, g_{n}\right\}$ of distinct elements of $G$ there is a group $H_{S} \in \mathcal{C}$ and a homomorphism $\phi_{S}: G \rightarrow H_{S}$, such that the images $\phi_{S}\left(g_{1}\right), \ldots, \phi_{S}\left(g_{n}\right)$ are all distinct in $H_{S}$.

The difference with the Classical Definition 1.2.1 is that the homomorphisms $\phi_{g}$ and $\phi_{S}$ are no longer required to be onto. The classes of (fully) residually finite and (fully) residually free groups $G$ are the same for both definitions. Indeed, the Definition 1.2.5 implies that there is a residual homomorphism $\phi: G \rightarrow \Gamma$ where $\Gamma$ is a finite (respectively, free) group; thus, $G \rightarrow \phi(G)$ is a residual epimomorphism where $\phi(G)$ is a finite (respectively, free) group, and so Definition 1.2.1 follows. The equivalence of the definitions for $\mathcal{C}$ being the class of torsion-free hyperbolic groups and $G$ being finitely generated is immediate from results of Ol'shanskii [Ol'93]. In Section 5.1, we show that the definitions are equivalent for the class of finitely generated toral relatively hyperbolic groups. The equivalence of the definitions is an immediate corollary of the following theorem.

Theorem 1.2.6. Let $\Gamma$ be a group in $\mathscr{R}$. Then every finitely generated subgroup of $\Gamma$ is a fully residually- $\mathscr{R}$ group in the sense of Definition 1.2.1.

The second change that one makes to extend the algebraic geometry over free groups to (relatively) hyperbolic groups, is that one's attention restricts to homomorphisms into a fixed group $\Gamma$ from the given class. Accordingly, the terminology changes to (fully) residually- $\Gamma$ groups and $\Gamma$-limit groups.

In this context, $\Gamma$-limit groups are precisely finitely generated fully residually- $\Gamma$ groups, when the group $\Gamma$ is either hyperbolic, as was shown by Sela in Sel09 for the torsion-free case and by Reinfeldt and Weidmann in [RW10] for all hyperbolic groups, or $\Gamma \in \mathscr{R}$, as was proved by Groves in Gro05]. Yet more generally, the same result holds if $\Gamma$ is an equationally Noetherian group, as was proved by Ould Houcine in [Hou07]. 
Note that usually, finitely generated (fully) residually-C $\mathcal{C}$ groups do not have to be in $\mathcal{C}$. For instance, infinite linear groups are residually finite [Mal40, and finitely generated free abelian groups $\mathbb{Z}^{n}$ are fully residually free by taking a projection homomorphism from $\mathbb{Z}^{n}$ to a component $\mathbb{Z}$ and applying Theorem 1.2 .2 . The former example can be illustrated by considering a homomorphism between the special linear groups of degree $n$ over $\mathbb{Z}$ and $\mathbb{Z}_{p}$, i.e., $S L(n, \mathbb{Z}) \rightarrow S L\left(n, \mathbb{Z}_{p}\right)$ where $p$ is a prime number so that for a given nontrivial matrix $A$ in $S L(n, \mathbb{Z})$ the image of $A$ is nontrivial. However, for some classes $\mathcal{C}$, a group $G$ is fully residually-C $\mathcal{C}$ if and only if $G \in \mathcal{C}$. An example is the class $\mathcal{A}$ of all abelian groups. Indeed, let a group $G$ be fully residually- $\mathcal{A}$, and suppose there are $a, b \in G$ such that $[a, b] \neq 1$. Clearly, both $a$ and $b$ are nontrivial. Then there is an abelian group $H$ and a homomorphism $\phi: G \rightarrow H$ such that $\phi(a), \phi(b)$ and $\phi([a, b])=[\phi(a), \phi(b)]$ are all nontrivial in $H$, which is a contradiction. It follows that $G$ is fully residually- $\mathcal{A}$ if and only if $G \in \mathcal{A}$. This analogue will not hold for the class of (relatively) hyperbolic groups. For example, a free rank 2 abelian group is residually hyperbolic; however, it does not embed into any hyperbolic group. In Section 5.3, we show that the class of finitely generated toral relatively hyperbolic groups is closed under extensions of centralizers (see Definition 5.3.1) and deduce the following theorem from a statement proved by Kharlampovich and Miasnikov in [KM12].

Theorem 1.2.7. Let $\Gamma$ be a finitely generated toral relatively hyperbolic group, and let $G$ be a finitely generated fully residually- $\Gamma$ group. Then $G$ embeds into a toral relatively hyperbolic group.

Note that finitely generated fully residually free groups are toral relatively hyperbolic; this follows from combination theorems proved by Dahmani [Dah03] and Alibegovič Ali05. On the other hand, neither this latter statement, nor even the conclusion of Theorem 1.2.7 holds for torsion-free finitely generated fully residually- $\mathcal{H}$ groups, where $\mathcal{H}$ is the class of hyperbolic groups. Indeed, there are Baumslag-Solitar fully residually- $\mathcal{H}$ groups that do not embed into any group in $\mathscr{R}$; please see Section 5.4 for details.

\subsection{Outline of the thesis}

The thesis is organized as follows. Chapter 2 introduces background and notation. Chapter 3 recalls some properties of relatively hyperbolic groups. Chapter 4 provides two efficient algorithms solving the word and conjugacy problems. In particular, the proofs of Theorems 1.1.3 and 1.1.5 are explained in Chapter 4. Chapter 5 proves the equivalence of Definitions 1.2.1 and 1.2.5. Theorem 1.2.4, a generalization of Theorem 1.2.2 by Baumslag [Bau67] to $\mathscr{R}$, is proved in Chapter 5, and the proof of Theorem 1.2.7 is explained in Chapter 5. The last chapter of the thesis consists of future research directions. 


\section{Chapter 2}

\section{Preliminaries}

In this chapter, we provide some definitions and basic facts related to the main part of this thesis; we also introduce the notation which will be used throughout this thesis.

\subsection{Cayley graphs}

We begin with the definition of a Cayley graph. Let $G$ be a group; and let $S$ be a generating set of $G$, denoted by $G=\langle S\rangle$. In this thesis, we always assume that the generating set does not contain the identity.

The Cayley graph of $G$ with respect to $S$, denoted by $\Gamma(G, S)$, is a directed labelled graph with the vertex set $\mathcal{V}=G$ and the edge set $\mathcal{E}=\mathcal{V} \times S=\{(g, s) \mid g \in G, s \in S\}$. Each edge $e=(g, s)$ goes from $g \in \mathcal{V}$ to $g s \in \mathcal{V}$ and has the label $\phi(e)=s$; we denote the origin and terminus (or endpoints) of $e$ by $e_{-}$and $e_{+}$, respectively. We assign length 1 to each edge of $\Gamma(G, S)$.

Let $\omega=e_{1} e_{2} \cdots e_{n}$ be a path in $\Gamma(G, S)$, where $e_{i} \in \mathcal{E}$ for all $i=1, \cdots, n$ and $e_{j_{+}}=e_{j+1}$ for all $j=1, \cdots, n-1$. The number $n$ of edges on the path $\omega$, denoted by $l_{\Gamma(G, S)}(\omega)$, is called the length of $\omega$; if the context is clear, we denote by $l(\omega)$. We say that $\omega$ is an empty path if $\omega$ does not contain an edge and has length 0 , and that a subpath of $\omega$ is a path of the form $e_{i} e_{i+1} \cdots e_{j}$ for some $1 \leq i \leq j \leq n$. If $x$ and $y$ are vertices in $\omega$, we denote by $\omega_{x, y}$ a subpath of $\omega$ from $x$ to $y$. The Cayley graph $\Gamma(G, S)$ can be made into a metric space by defining the distance $d_{\Gamma(G, S)}$ between any two vertices in $\mathcal{V}$ to be the minimal length of paths joining them; if the context is clear, we denote this distance by $d$. We denote the origin and terminus (or endpoints) of $\omega$ by $\omega_{-}=e_{1-}$ and $\omega_{+}=e_{n+}$. If $\omega_{-}=\omega_{+}$, we say that $\omega$ is a closed path. The label of $\omega$ is denoted by $\phi(\omega)=\phi\left(e_{1}\right) \phi\left(e_{2}\right) \cdots \phi\left(e_{n}\right)$. Note that $\phi(\omega)$ is a word as a product of generators, i.e., over the alphabet $S^{ \pm 1}=S \cup S^{-1}$ representing an element in $G$. We denote by $\left(S^{ \pm 1}\right)^{*}$ the free monoid on $S^{ \pm 1}$. Then $\phi(\omega) \in\left(S^{ \pm 1}\right)^{*}$. If $\mathrm{w}=\phi(\omega)$, we say that $l_{\Gamma(G, S)}(\omega)$ is the length of $\mathrm{w}$, denoted by $\|\mathrm{w}\|_{S}$; if the context is clear, we denote by $\|\mathrm{w}\|$. We say that $\mathrm{w}$ is freely reduced if the path $\omega$ does not contain a subpath of the form $e_{i} e_{i+1}^{-1}$ or $e_{i}^{-1} e_{i+1}$ for some $1 \leq i \leq n-1$.

Example 2.1.1. See Figure 2.1 for Cayley graphs of the following groups:

1. the finite group of order 2 , denoted by $\mathbb{Z}_{2}=\left\langle c \mid c^{2}=1\right\rangle$,

2. the free abelian group of rank 2 , denoted by $\mathbb{Z} \times \mathbb{Z}=\left\langle x, y \mid[x, y]=x y x^{-1} y^{-1}=1\right\rangle$,

3. the free group generated by $\{a, b\}$, denoted by $F(a, b)=\mathbb{Z} * \mathbb{Z}=\langle a, b \mid-\rangle$. 

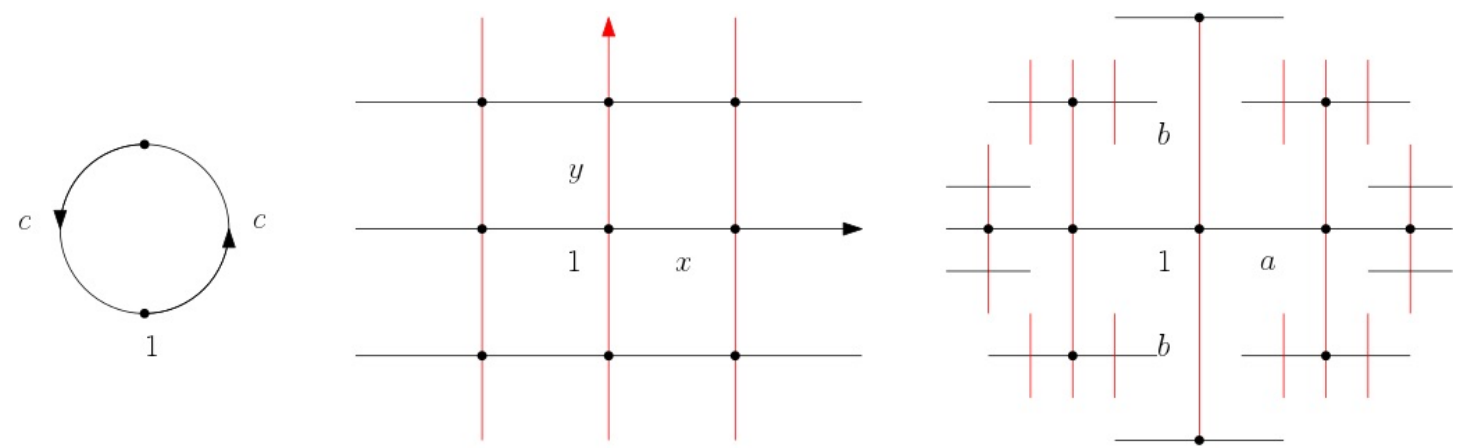

Figure 2.1: From left to right, $\mathbb{Z}_{2}, \mathbb{Z} \times \mathbb{Z}$ and $F(a, b)$

Definition 2.1.2. Let $\omega$ be a path in $\Gamma(G, S)$; and let $\omega^{\prime}$ be a subpath of $\omega$.

1. We say that $\omega$ is a geodesic if $l(\omega)=d\left(\omega_{-}, \omega_{+}\right)$. We denote by $\left[\omega_{-}^{\prime}, \omega_{+}^{\prime}\right]$ a geodesic connecting vertices $\omega_{-}^{\prime}$ and $\omega_{+}^{\prime}$.

2. Let $\lambda \geq 1$; and let $\epsilon \geq 0$. We say that $\omega$ is a $(\lambda, \epsilon)$-quasigeodesic if for any subpath $\omega^{\prime}, l\left(\omega^{\prime}\right) \leq \lambda d\left(\omega_{-}^{\prime}, \omega_{+}^{\prime}\right)+\epsilon$.

3. Let $L>1$ be an integer. We say that $\omega$ is a $L$-local geodesic if for any subpath $\omega^{\prime}$ such that $l\left(\omega^{\prime}\right) \leq L, \omega^{\prime}$ is geodesic.

The above Definition 2.1.2 implies the following statement.

Lemma 2.1.3. Any subpath of a geodesic, $(\lambda, \epsilon)$-quasigeodesic or L-local geodesic in $\Gamma(G, S)$ is a geodesic, $(\lambda, \epsilon)$-quasigeodesic or L-local geodesic, respectively. Moreover, any geodesic is a L-local geodesic for any $L$.

Let $\alpha$ and $\beta$ be paths in $\Gamma(G, S)$. If $\beta_{-}=\alpha_{+}$, we denote by $\alpha \beta$ the path which is the concatenation of $\alpha$ and $\beta$.

Lemma 2.1.4. Osi06c, Lemma 3.4] Let $\omega=\alpha \beta$ or $\beta \alpha$ be a path in $\Gamma(G, S)$, where $\alpha$ is $a(\lambda, \epsilon)$-quasigeodesic and $l(\beta) \leq k$ for some constant $k$. Then $\omega$ is $a\left(\lambda, \epsilon^{\prime}\right)$-quasigeodesic, where $\epsilon^{\prime}=\epsilon+(\lambda+1) k$.

Proof. Without loss of generality, we assume that $\omega=\alpha \beta$. Let $\omega^{\prime}$ be a subpath of $\omega$. Then there are three cases to discuss.

Firstly, if $\omega^{\prime}$ is a subpath of $\alpha$, then $l\left(\omega^{\prime}\right) \leq \lambda d\left(\omega^{\prime}{ }_{-}, \omega_{+}^{\prime}\right)+\epsilon$ because $\alpha$ is $(\lambda, \epsilon)$ quasigeodesic. Secondly, if $\omega^{\prime}$ is a subpath of $\beta$, then $l\left(\omega^{\prime}\right) \leq l(\beta) \leq k$. Lastly, if $\omega^{\prime} \in \alpha$ and $\omega_{+}^{\prime} \in \beta$, then $\omega^{\prime}=\alpha^{\prime} \beta^{\prime}$, where $\alpha^{\prime}$ and $\beta^{\prime}$ are subpaths of $\alpha$ and $\beta$, respectively; it follows that

$$
\begin{aligned}
l\left(\omega^{\prime}\right) & =l\left(\alpha^{\prime}\right)+l\left(\beta^{\prime}\right) \\
& \leq\left(\lambda d\left(\alpha_{-}^{\prime}, \alpha^{\prime}+\right)+\epsilon\right)+k \\
& \leq \lambda\left(d\left(\alpha^{\prime}{ }_{-}, \beta_{+}^{\prime}\right)+d\left(\beta_{-}^{\prime}, \beta_{+}^{\prime}\right)\right)+(\epsilon+k) \\
& \leq \lambda\left(d\left(\alpha^{\prime}, \beta_{+}^{\prime}\right)+k\right)+(\epsilon+k) \\
& =\lambda d\left(\omega_{-}^{\prime}, \omega_{+}\right)+[\epsilon+(\lambda+1) k] .
\end{aligned}
$$

All together, $\omega$ is a $(\lambda, \epsilon+(\lambda+1) k)$-quasigeodesic, and the statement is proven. 


\subsection{Hyperbolic groups}

We start by giving a definition of a hyperbolic group.

Definition 2.2.1. A geodesic metric space $X$ is $\delta$-hyperbolic if there exists $\delta \geq 0$ such that for any geodesic triangle $T$ in $X$, any side of $T$ belongs to the union of the closed $\delta$-neighbourhoods of the other two sides. A group is called a hyperbolic group if it has a finite generating set such that its Cayley graph with respect to the generating set is a $\delta$-hyperbolic metric space for some $\delta \geq 0$.

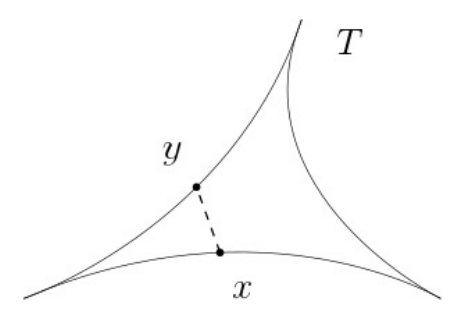

Figure 2.2: A geodesic triangle $T$ in a $\delta$-hyperbolic geodesic metric space.

Recall that a $(\lambda, \epsilon)$-quasi-isometry between metric spaces is a map $f: X \rightarrow Y$ such that, for some $\lambda, \epsilon, \epsilon^{\prime}>0$ :

1. $\frac{1}{\lambda} d_{X}\left(x_{1}, x_{2}\right)-\epsilon \leq d_{Y}\left(f\left(x_{1}\right), f\left(x_{2}\right)\right) \leq \lambda d_{X}\left(x_{1}, x_{2}\right)+C$ for all $x_{1}, x_{2} \in X$;

2. for all $y \in Y$, there is $x \in X$ such that $d_{Y}(y, f(x)) \leq \epsilon^{\prime}$.

Remark 2.2.2. If $S$ and $T$ are two finite generating sets of a finitely generated group $G$, the Cayley graphs $\Gamma(G, S)$ with respect to $S$ and $\Gamma(G, T)$ with respect to $T$ are quasi-isometric. Indeed, if $f: \Gamma(G, S) \rightarrow \Gamma(G, T)$ is the identity mapping, then $\Gamma(G, S)$ and $\Gamma(G, T)$ are $(\lambda, 0)$-quasi-isometric, where $\lambda=\max \left\{\max _{s \in S}\|s\|_{T}, \max _{t \in T}\|t\|_{S}\right\}$. It follows from the fact [GdLH, Chapter 5] that if $\Gamma(G, S)$ is hyperbolic, so is $\Gamma(G, T)$. Thus, hyperbolicity is independent of choices for generating sets for $G$.

\section{Example 2.2.3.}

1. Any finite group is hyperbolic since its Cayley graph is $\delta$-hyperbolic, where $\delta$ is the number of elements in the group.

2. Any finitely generated free group is hyperbolic since its Cayley graph with respect to a free basis is 0-hyperbolic.

3. The free abelian group of rank $2, \mathbb{Z} \times \mathbb{Z}$, is not hyperbolic. Indeed, from the second Example 2.1.1. it is impossible to find an universal constant $\delta$ to bound all geodesic triangles. Moreover, no hyperbolic group has a subgroup isomorphic to $\mathbb{Z} \times \mathbb{Z}$. This is due to the well-known fact [BH99, Corollary 3.10, Chapter III.Г] that the centralizer $C(h)$ of an infinite order element $h$ in a hyperbolic group is such that the quotient $C(h) /\langle h\rangle$ is finite.

Lemma 2.2.4. Let $X$ be a $\delta$-hyperbolic geodesic space and let $Q$ be a geodesic quadrangle in $X$, that is, four sides of $Q$ are geodesics. Then each side of $Q$ is contained in the $2 \delta$-neighbourhood of the other three sides. 
Proof. Let $Q=\gamma_{1} \gamma_{2} \gamma_{3} \gamma_{4}$; and let $\gamma=\left[\gamma_{1_{-}}, \gamma_{2+}\right]$. Let $x$ be a vertex in $\gamma_{1}$. Since $X$ is a $\delta$-hyperbolic geodesic space, there exists a vertex $y$ lying on $\gamma_{2}$ or $\gamma$ such that $d(x, y) \leq \delta$. If $y$ is a vertex in $\gamma$, there is a vertex $z$ lying on $\gamma_{3}$ or $\gamma_{4}$ such that $d(y, z) \leq \delta$. See Figure 2.3 . Therefore, the result follows.

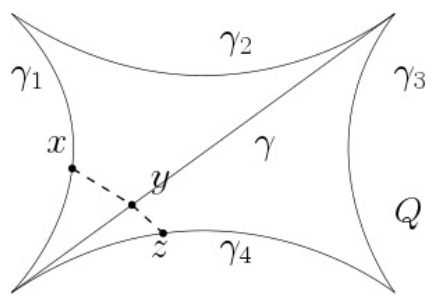

Figure 2.3: A quadrangle $Q$ in $X$

The following statement is from Theorem 1.13 Chapter III.H of [BH99] on Page 405; we modify it in terms of our language.

Lemma 2.2.5. [BH99, Theorem 1.13 Chapter III.H] Let $X$ be a $\delta$-hyperbolic geodesic space and let $\omega$ be a L-local geodesic in $X$, where $L>8 \delta$. Then:

(1) $\omega$ is contained in the $2 \delta$-neighbourhood of any geodesic $\left[\omega_{-}, \omega_{+}\right]$,

(2) $\left[\omega_{-}, \omega_{+}\right]$is contained in the $3 \delta$-neighbourhood of $\omega$, and

(3) $\omega$ is a $(\lambda, \epsilon)$-quasigeodesic, where $\epsilon=2 \delta$ and $\lambda=\frac{L+4 \delta}{L-4 \delta}$.

Corollary 2.2.6. With the notation and assumptions of Lemma 2.2.5, if $L=8 \delta+1$, then $\omega$ is a $(3,2 \delta)$-quasigeodesic in $X$.

Proof. Note that $\frac{L+4 \delta}{L-4 \delta}=\frac{12 \delta+1}{4 \delta+1}<\frac{12 \delta+3}{4 \delta+1}=3$. By Lemma 2.2.5 (3), the result follows.

Corollary 2.2.7. [BH99, Corollary 1.14 Chapter III.H] With the notation and assumptions of Lemma 2.2.5, if $\omega$ is non-empty, then $\omega$ is a path with different endpoints, i.e., $\omega_{-} \neq \omega_{+}$.

Let us recall a Dehn presentation of a group.

Definition 2.2.8 (Dehn presentation). A finite presentation $\langle S \mid R=1, R \in \mathcal{R}\rangle$ of a group $G$ is called a Dehn presentation if for every nontrivial freely reduced word $w$ in $\left(S^{ \pm 1}\right)^{*}$ representing the identity in $G$, the word $w$ contains more than half of some word in $\mathcal{R}$, that is, there is a relator $R=u v \in \mathcal{R}$ with $\|v\|<\|u\|$ such that $w=w_{1} u w_{2}$.

Definition 2.2.9 (Dehn's algorithm). A group is said to have a Dehn's algorithm if it has a Dehn presentation. More precisely, it can be described as follows. Let $\langle X \mid \mathcal{R}\rangle$ be a finite presentation of a group $G$; and let $w$ be a non-empty word in $\left(X^{ \pm 1}\right)^{*}$. One looks for a subword $u$ that is a subword of a relator $R=u v \in \mathcal{R}$ such that $\|u\|>\|v\|$. If there is no such subword, one terminates and outputs that $w$ does not represent the identity; otherwise, one replaces $u$ with $v^{-1}$ and repeats the search for a subword of the edited word $w^{\prime}$, where $w^{\prime}$ represents the same element as $w$ such that $\left\|w^{\prime}\right\|<\|w\|$. After at most $\|w\|$ steps, one will have either reduced to the empty word or verified that $w$ does not represent the identity.

The following theorem is due to Gromov [Gro87] and a proof can be found in [BH99, Theorem 2.6 Chapter III.H].

Theorem 2.2.10. [BH99, Theorem 2.6 Chapter III.H] A group is hyperbolic if and only if it admits a Dehn presentation. 


\subsection{Relatively hyperbolic groups}

There are several definitions of a relatively hyperbolic group. In this section, we will give two definitions of a relatively hyperbolic group which are introduced by Osin Osi06c and Farb [Far98]; we also provide some preliminary results to this thesis.

\subsubsection{The definition of Osin}

This subsection is based on the work of Osin in Osi06c]. Let $G$ be a group, $\left\{H_{\lambda}, \lambda \in \Lambda\right\}$ be a collection of subgroups of $G$, and $X$ be a subset of $G$. We say that $X$ is a relative generating set of $G$ with respect to $\left\{H_{\lambda}, \lambda \in \Lambda\right\}$ if $G$ is generated by the set $\left(\bigcup_{\lambda \in \Lambda} H_{\lambda}\right) \cup X$ where $X$ is assumed to be symmetrized, i.e., $X=X^{-1}$. In this situation, $G$ can be regarded as the quotient group of the free product

$$
F=\left(*_{\lambda \in \Lambda} \widetilde{H}_{\lambda}\right) * F(X),
$$

where each $\widetilde{H}_{\lambda}$ is isomorphic to $H_{\lambda}$ and $F(X)$ is the free group with the basis $X$. We denote by $\mathcal{H}$ the disjoint union

$$
\mathcal{H}=\bigsqcup_{\lambda \in \Lambda}\left(\widetilde{H}_{\lambda} \backslash\{1\}\right)
$$

Note that the group $F$ is generated by the set $X \cup \mathcal{H}$. For every $\lambda \in \Lambda$, we denote by $\mathcal{S}_{\lambda}$ the set of all words in the alphabet $\widetilde{H}_{\lambda} \backslash\{1\}$ that represent the identity of $F$. So, the group $G$ can be defined by the presentation

$$
\left\langle X, \mathcal{H} \mid S=1, S \in \bigcup_{\lambda \in \Lambda} \mathcal{S}_{\lambda}, R=1, R \in \mathcal{R}\right\rangle
$$

where $\mathcal{R}$ is a set of words in $(X \cup \mathcal{H})^{*}$ such that $F /\langle\langle\mathcal{R}\rangle\rangle \cong G$, i.e., the kernel of the homomorphism $F \rightarrow G$ is the normal closure of $\mathcal{R}$.

Definition 2.3.1 (Relative presentation). The presentation $(2.3)$ is called the relative presentation of $G$ with respect to $\left\{H_{\lambda}, \lambda \in \Lambda\right\}$. For brevity, we write

$$
\left\langle X, H_{\lambda}, \lambda \in \Lambda \mid R=1, R \in \mathcal{R}\right\rangle .
$$

Definition 2.3.2. The relative presentation (2.4) is finite if the sets $X$ and $\mathcal{R}$ are finite. The group $G$ is called finitely presented relative to $\left\{H_{\lambda}, \lambda \in \Lambda\right\}$ if $G$ has a finite relative presentation with respect to $\left\{H_{\lambda}, \lambda \in \Lambda\right\}$.

Before going to introduce the definition of a relatively hyperbolic group, we need the following terminology, which will play an important role in the thesis.

Definition 2.3.3 $\left(H_{\lambda}\right.$-subword). Given a word $\mathrm{w} \in(X \cup \mathcal{H})^{*}$, we say that a subword $\mathrm{v}$ of $\mathrm{w}$ is a $H_{\lambda}$-subword if $\mathrm{v}$ consists of letters from $\widetilde{H}_{\lambda}$. A $H_{\lambda}$-subword of $\mathrm{w}$ is called a $H_{\lambda}$-syllable if it is maximal, i.e., it is not contained in a bigger $H_{\lambda}$-subword of $\mathrm{w}$.

Remark 2.3.4. For any $\lambda \in \Lambda$, the length of every $H_{\lambda}$-subword $\mathrm{v}$ of $\mathrm{w}$ with respect to $X \cup \mathcal{H}$ is 1, i.e., $\|\mathrm{v}\|_{X \cup \mathcal{H}}=1$.

For the rest of this subsection, suppose that the group $G$ is given by the relative presentation (2.3). Let $\Gamma(G, X \cup \mathcal{H})$ be the Cayley graph of $G$ with respect to the generating set $X \cup \mathcal{H}$. 
Definition 2.3.5 $\left(H_{\lambda}\right.$-component). Let $\omega$ be a path in $\Gamma(G, X \cup \mathcal{H})$. A subpath $\eta$ of $\omega$ is called a $H_{\lambda}$-subpath if $\phi(\eta)$ is a $H_{\lambda}$-subword of the word $\phi(\omega)$. A component (or more precisely a $H_{\lambda}$-component) of $\omega$ is a $H_{\lambda}$-subpath such that $\phi(\eta)$ is a $H_{\lambda}$-syllable of the word $\phi(\omega)$.

Definition 2.3.6 (Connected component). Two $H_{\lambda}$-components $\eta_{1}, \eta_{2}$ of a path $\omega$ in $\Gamma(G, X \cup \mathcal{H})$ are called connected if there exists a path $\sigma$ in $\Gamma(G, X \cup \mathcal{H})$ that connects some vertex $v_{1}$ of $\eta_{1}$ to some vertex $v_{2}$ of $\eta_{2}$ and $\phi(\sigma)$ is a word consists of letters from $\widetilde{H}_{\lambda}$. (In algebraic terms, this means that $v_{1}$ and $v_{2}$ belong to the same coset $g H_{\lambda}$.)

Definition 2.3.7 (Isolated component). An $H_{\lambda}$-component $\eta$ of a path $\omega$ in $\Gamma(G, X \cup \mathcal{H})$ is called isolated if no (distinct from $\eta$ ) $H_{\lambda}$-component is connected to $\eta$.

Example 2.3.8. Consider the free abelian group of rank $2, \mathbb{Z} \times \mathbb{Z}=\langle x, y \mid[x, y]=1\rangle$. Let $H=\langle x\rangle$ and consider the word $\mathrm{w}=x y x^{2} y^{-1} x^{3}$. Then $x, x^{2}$ and $x^{3}$ are $H$-syllables of $\mathrm{w}$, and $\|\mathrm{w}\|_{X \cup H}=5$ where $X=\left\{y^{ \pm 1}\right\}$. See Figure 2.4 for the path $\omega$ in $\Gamma=\Gamma(\mathbb{Z} \times \mathbb{Z}, X \cup H)$ such that $\phi(\omega)=\mathrm{w}$. Then $l_{\Gamma}(\omega)=5$. Let $\eta_{1}$ and $\eta_{2}$ be the subpaths of $\omega$ labeled by $x$ and

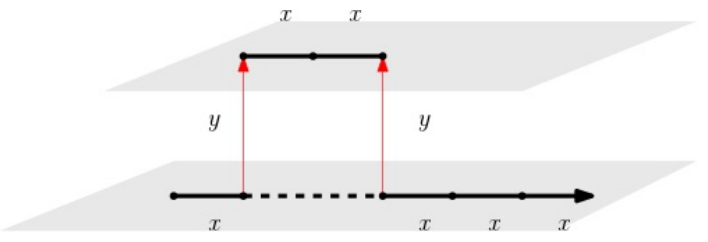

Figure 2.4: The path $\omega \in \Gamma$ with $\phi(\omega)=\mathrm{w}$

$x^{3}$, respectively; and let $\zeta$ be the subpath of $\omega$ with $\phi(\zeta)=x^{2}$. Then the $H$-components $\eta_{1}$ and $\eta_{2}$ are connected; the $H$-component $\zeta$ is isolated.

Now, we introduce the definition of a relatively hyperbolic group.

Given a word w in $(X \cup \mathcal{H})^{*}$ such that w represents the identity of $G$, there exists an expression

$$
\mathrm{w}={ }_{F} \prod_{i=1}^{k} f_{i}^{-1} R_{i}^{ \pm 1} f_{i}
$$

with the equality in the group $F$ defined in (2.1), where $R_{i} \in \mathcal{R}, f_{i} \in F$ for $i=1, \cdots, k$. The smallest possible $k$ is called the relative area of $\mathrm{w}$ and is denoted by $\operatorname{Area}^{\text {rel }}(\mathrm{w})$.

Definition 2.3.9. We say that $G$ is hyperbolic relative to $\left\{H_{\lambda}, \lambda \in \Lambda\right\}$ if the following two conditions hold:

(1) $G$ is finitely presented relative to $\left\{H_{\lambda}, \lambda \in \Lambda\right\}$; and

(2) there is a constant $K>0$ such that for any word w in $(X \cup \mathcal{H})^{*}$, representing the identity of $G$, we have $\operatorname{Area}^{r e l}(\mathrm{w}) \leq K\|\mathrm{w}\|_{X \cup \mathcal{H}}$, where $\mathcal{H}$ is defined in 2.2).

\subsubsection{The definition of Farb}

Throughout this subsection, we denote by $G$ a group finitely generated by the set $S$, $\Gamma=\Gamma(G, S)$ the Cayley graph of $G$ with respect to $S$, and denote by $\mathcal{H}=\left\{H_{1}, \cdots, H_{m}\right\}$ a finite set of finitely generated subgroups of $G$; we assume that $H_{i}=\left\langle S_{i}\right\rangle$ and $S=$ $S_{0} \sqcup S_{1} \sqcup \cdots \sqcup S_{m}$; we also denote by $\Gamma_{i}$ the Cayley graph of $H_{i}$ with respect to $S_{i}$ for all $1 \leq i \leq m$. 
Remark 2.3.10. Because of the assumption that $H_{i}=\left\langle S_{i}\right\rangle$ and $S=S_{0} \sqcup S_{1} \sqcup \cdots \sqcup S_{m}$, the definitions of components, connected components and isolated components can be defined as Osin's definitions in the previous subsection 2.3.1 replacing $\Gamma(G, X \cup \mathcal{H})$ by $\Gamma(G, S)$. See the following definition.

Definition 2.3.11. Let $\mathcal{A}_{i}=S_{i}^{ \pm 1}$ for all $0 \leq i \leq m$.

- Let $\eta$ be a subpath of a path $\omega$ in $\Gamma$. If the word $\phi(\eta) \in \mathcal{A}_{t}^{*}$ for some $1 \leq t \leq m$, then we say that $\eta$ is a $\Gamma_{t}$-subpath of $\omega$. If no $\Gamma_{t}$-subpath of $\omega$ contains $\eta$ as a proper subpath, $\eta$ is called a $\Gamma_{t}$-component (or component) of $\omega$.

- Let $\eta_{1}$ and $\eta_{2}$ be two distinct $\Gamma_{t}$-components of $\omega$, i.e., $\phi\left(\eta_{1}\right) \neq \phi\left(\eta_{2}\right)$. If there is a path $\sigma$ in $\Gamma$ connecting a vertex of $\eta_{1}$ and a vertex of $\eta_{2}$ such that the word $\phi(\sigma) \in \mathcal{A}_{t}^{*}$, then we say that $\eta_{1}$ and $\eta_{2}$ are connected.

- A component $\eta$ of a path $\omega$ in $\Gamma$ is called isolated if no other component $\sigma$ of $\omega$ is connected to $\eta$, where $\phi(\eta) \neq \phi(\sigma)$.

Let $\widehat{\Gamma}(G, \mathcal{H})$ be a new graph constructed from $\Gamma$ as follows: for each $H_{i} \in \mathcal{H}$ in $G$, add a vertex $v\left(g H_{i}\right)$ for every left coset $g H_{i}$ of $H_{i}$, and connect $v\left(g H_{i}\right)$ with each element in $g H_{i}$ by an edge of length $\frac{1}{2}$. We call $\widehat{\Gamma}=\widehat{\Gamma}(G, \mathcal{H})$ the coned-off Cayley graph of $G$ with respect to $\mathcal{H}$, and give $\widehat{\Gamma}$ the metric. Note that $\widehat{\Gamma}$ is not a proper metric space because some closed ball may not be compact, that is, some vertices from a closed ball are not necessarily lying within some bounded $\Gamma$-distance of each other. See Figure 2.5 for a path in $\widehat{\Gamma}$ connecting vertices $x$ and $y$ in the coset $g H_{i}$.

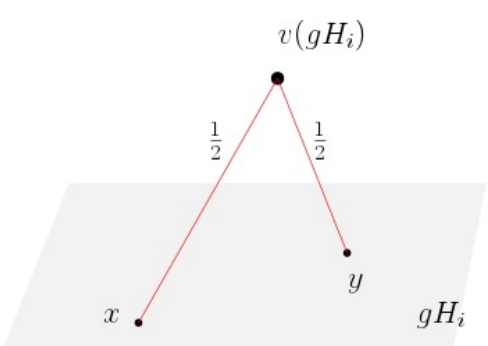

Figure 2.5: A path connecting vertices $x$ and $y$ in $\widehat{\Gamma}$

Definition 2.3.12 (Weakly hyperbolic group). The group $G$ is weakly hyperbolic relative to $\mathcal{H}$ if the coned-off Cayley graph $\widehat{\Gamma}$ of $G$ with respect to $\mathcal{H}$ is a hyperbolic metric space.

Example 2.3.13. The group $\mathbb{Z} \times \mathbb{Z}=\langle x, y \mid[x, y]=1\rangle$ is weakly hyperbolic relative to $H=\langle y\rangle$ as $\widehat{\Gamma}(\mathbb{Z} \times \mathbb{Z}, H)$ is quasi-isometric to a 0 -hyperbolic metric space. See Figure 2.6.

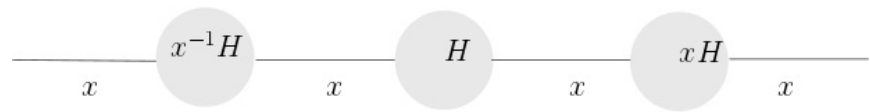

Figure 2.6: $\widehat{\Gamma}(\mathbb{Z} \times \mathbb{Z}, H)$ 
Remark 2.3.14. The above definition depends on the choice of a generating set for $G$. Nevertheless, the weak relative hyperbolicity of $G$ is independent of choices of generating sets for $G$ and for each $H_{i} \in \mathcal{H}$ [Far98, Corollary 3.2].

Throughout the rest of this subsection, we denote by $\widehat{\Gamma}$ the coned-off Cayley $\widehat{\Gamma}(G, \mathcal{H})$ of $G$ with respect to $\mathcal{H}$. Let $\omega$ be a path in $\Gamma$. We denote by $\widehat{\omega}$ the path in $\widehat{\Gamma}$ obtained from $\omega$ in $\Gamma$ in the following way: for each $H_{i} \in \mathcal{H}$ in $G$, we replace every $H_{i}$-component $\eta$ of $\omega$ by an edge of length 1 in $\widehat{\Gamma}$ from $\eta_{-}$to $\eta_{+}$passing through the coned vertex $v\left(g H_{i}\right)$. Note that $\widehat{\eta}_{-}=\eta_{-}$and $\widehat{\eta}_{+}=\eta_{+}$and that $d_{\widehat{\Gamma}}\left(\eta_{-}, \eta_{+}\right)=1$. We say that $\eta_{-}$is an entering vertex of $\widehat{\omega}$ in the coset $g H_{i}$ and $\eta_{+}$is an exiting vertex of $\widehat{\omega}$ in the coset $g H_{i}$. If $\widehat{\omega}$ passes through some coned vertex $v\left(g H_{i}\right)$, we say that $\omega$ (or $\widehat{\omega}$ ) penetrates the coset $g H_{i}$.

Definition 2.3.15 (Backtracking). A path $\omega$ in $\Gamma$ (or $\widehat{\omega}$ in $\widehat{\Gamma}$ ) is said to be a path without backtracking if, for every coset $g H_{i}$ which $\omega$ (or $\widehat{\omega}$, respectively) penetrates, $\omega$ (or $\widehat{\omega}$, respectively) never returns to $g H_{i}$ after leaving $g H_{i}$.

Throughout this thesis, given a path $\widehat{\omega}$ in $\widehat{\Gamma}$, we denote by $\omega$ a path in $\Gamma$ such that $\widehat{\omega}$ is obtained from $\omega$.

Definition 2.3.16 (Bounded Coset Penetration property). The pair $(G, \mathcal{H})$ is said to satisfy the Bounded Coset Penetration property (or BCP property for short) if for every $\lambda \geq 1$, there is a constant $C=C(\lambda)>0$ so that if $\widehat{\alpha}$ and $\widehat{\beta}$ are $(\lambda, 0)$-quasigeodesics in $\widehat{\Gamma}$ without backtracking with $\widehat{\alpha}_{-}=\widehat{\beta}_{-}$and $d_{\Gamma}\left(\alpha_{+}, \beta_{+}\right) \leq 1$, then the following condition holds:

1. If $\widehat{\alpha}$ penetrates a coset $g H_{i}$ but $\widehat{\beta}$ does not penetrate $g H_{i}$ then $\widehat{\alpha}$ travels a $\Gamma$-distance of at most $C$ in $g H_{i}$.

2. If both $\widehat{\alpha}$ and $\widehat{\beta}$ penetrate a coset $g H_{i}$, then the entering vertices of $\widehat{\alpha}$ and $\widehat{\beta}$ in $g H_{i}$ lie a $\Gamma$-distance of at most $C$ from each other; similarly for the exiting vertices in $g H_{i}$.

Definition 2.3.17 (Strong relative hyperbolicity). The group $G$ is hyperbolic relative to $\mathcal{H}$ in the strong sense, if $G$ is weakly hyperbolic relative to $\mathcal{H}$, and the pair $(G, \mathcal{H})$ satisfies the BCP property.

Example 2.3.18. With the notation of Example 2.3.13, the pair $(\mathbb{Z} \times \mathbb{Z}, H)$ does not satisfy the $B C P$ property; and so, the group $\mathbb{Z} \times \mathbb{Z}$ is not hyperbolic relative to $\mathbb{Z}$ in the strong sense. Indeed, the paths $\widehat{\alpha}$ and $\widehat{\beta}$ in $\widehat{\Gamma}$ such that $\phi(\alpha)=x^{n}$ and $\phi(\beta)=y x^{n}$ are geodesics in $\widehat{\Gamma}$ without backtracking ending a $\Gamma$-distance 1 apart; however, it violates the first condition of the BCP property when $n$ is large enough. 


\section{Chapter 3}

\section{Some properties of relatively hyperbolic groups}

In this chapter, we collect some properties of relatively hyperbolic groups; and we obtain some consequences, which will be used later in the thesis.

\subsection{Equivalent definitions of relative hyperbolicity}

Compared to Farb's strong relative hyperbolicity, Osin has a more general notion of relative hyperbolicity, which allows infinitely many or possibly infinitely generated subgroups. However, if the group $G$ is finitely generated, then we have the following statements.

Theorem 3.1.1. Osi06c, Theorem 1.1] If the group $G$ is finitely generated and finitely presented relative to $\left\{H_{\lambda}, \lambda \in \Lambda\right\}$, then the collection of subgroups is finite and each subgroup $H_{\lambda}$ is finitely generated.

Remark 3.1.2. Dahmani and Guirardel proved in DG13 that if $G$ is finitely presented then each subgroup $H_{\lambda}$ is finitely presented.

The following result is a part of Theorem 6.10 from Osi06c].

Theorem 3.1.3. [Osi06c, Theorem 6.10] Let $G$ be a finitely generated group. Then $G$ is hyperbolic relative to $\left\{H_{\lambda}, \lambda \in \Lambda\right\}$ in the sense of Osin if and only if it satisfies the definition of Farb in the strong sense.

Definition 3.1.4. Let $G$ be a group hyperbolic relative to a collection of subgroups $\left\{H_{\lambda}, \lambda \in \Lambda\right\}$. We say that a subgroup $P_{\lambda}$ of $G$ is parabolic if $P_{\lambda}$ is a subgroup of $g H_{\lambda} g^{-1}$ for some $g \in G$ and $\lambda \in \Lambda$. An element $x$ of $G$ is called parabolic if it lies in some parabolic subgroup of $G$. Otherwise, $x$ is called hyperbolic.

Remark 3.1.5. By Definition 2.3.9, $G$ is hyperbolic relative to $\left\{H_{\lambda}, \lambda \in \Lambda\right\}$ if and only if $G$ is hyperbolic relative to $\left\{P_{\lambda}, \lambda \in \Lambda\right\}$, where $P_{\lambda}=g_{\lambda} H_{\lambda} g_{\lambda}^{-1}$ is a parabolic subgroup of $G$ for each $\lambda \in \Lambda$ and some $g_{\lambda} \in G$.

For the rest of this section, we assume that $G$ is a group finitely generated by $S$ and hyperbolic relative to parabolic subgroups $\mathcal{P}=\left\{P_{i}, 1 \leq i \leq m\right\}$ in the sense of Farb (see Subsection 2.3.2), where each $P_{i}=\left\langle S_{i}\right\rangle$ and $S=S_{0} \sqcup S_{1} \sqcup \cdots \sqcup S_{m}$. We denote by $\Gamma=\Gamma(G, S)$ the Cayley graph of $G$ with respect to $S, \Gamma_{i}=\Gamma\left(P_{i}, S_{i}\right)$ the Cayley graph of $P_{i}$ with respect to $S_{i}$ for all $1 \leq i \leq m$, and $\widehat{\Gamma}=\widehat{\Gamma}(G, \mathcal{P})$ the coned-off Cayley graph 
of $G$ with respect to $\mathcal{P}$. We denote by $\mathcal{A}=S^{ \pm 1}$ and $\mathcal{A}_{i}=S_{i}^{ \pm 1}$ for all $1 \leq i \leq m$. We conclude this section by giving the following lemmas.

Lemma 3.1.6. [Bum15, Lemma 3.7] Let $L=8 \delta+1$ for some $\delta \geq 1$; and let $\widehat{\omega}$ be a L-local geodesic in $\widehat{\Gamma}$. Then $\widehat{\omega}$ is a path without backtracking. In particular, every geodesic in $\widehat{\Gamma}$ is a path without backtracking.

Proof. Suppose that $\widehat{\omega}$ has a backtracking. Then $\omega$ contains a subpath $\eta \alpha$ in $\Gamma$, where $\eta$ is a $P_{i}$-component of $\omega$ penetrating the coset $g P_{i}$ for some $P_{i} \in \mathcal{P}$ and $g \in G$, and $\alpha$ is a non-empty subpath of $\omega$ such that there is a path $c$ in $\Gamma$ connecting the endpoints of $\alpha$ with $\phi(c) \in \mathcal{A}_{i}^{*}$ and that $\phi(\alpha) \notin \mathcal{A}_{i}^{*}$. Note that $d_{\widehat{\Gamma}}\left(\alpha_{-}, \alpha_{+}\right)=1$. See Figure 3.1. Since

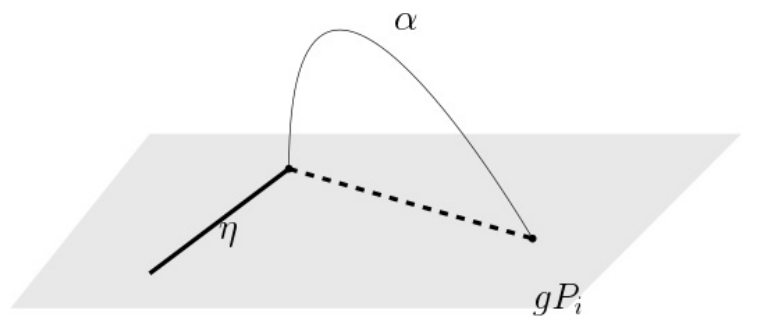

Figure 3.1: A backtracking

$\widehat{\omega}$ is a $L$-local geodesic in $\widehat{\Gamma}, \widehat{\eta \alpha}$ is a $L$-local geodesic in $\widehat{\Gamma}$. Note that $d_{\widehat{\Gamma}}\left(\widehat{\eta \alpha}_{-}, \widehat{\eta \alpha}_{+}\right)=1$. Since $L=8 \delta+1$, Corollary 2.2.6 implies that $\widehat{\eta \alpha}$ is a $(3,2 \delta)$-quasigeodesic in $\widehat{\Gamma}$; and so, $l_{\widehat{\Gamma}}(\widehat{\eta \alpha}) \leq 3 d_{\widehat{\Gamma}}\left(\widehat{\eta \alpha}_{-}, \widehat{\eta \alpha}_{+}\right)+2 \delta=3+2 \delta<L$ as $\delta \geq 1$. It follows that $\widehat{\eta \alpha}$ is a geodesic in $\widehat{\Gamma}$. Hence, $l_{\widehat{\Gamma}}(\widehat{\eta \alpha})=1$, which contradicts to $l_{\widehat{\Gamma}}(\widehat{\eta \alpha})=l_{\widehat{\Gamma}}(\widehat{\eta})+l_{\widehat{\Gamma}}(\widehat{\alpha}) \geq 2$. Therefore, $\widehat{\omega}$ is a path in $\widehat{\Gamma}$ without backtracking. Note that every geodesic in $\widehat{\Gamma}$ is a $L$-local geodesic in $\widehat{\Gamma}$ for any $L$. So, the second assertion follows.

Lemma 3.1.7. For any $\lambda \geq 1, \epsilon \geq 0$ and $k \geq 0$, there exists a constant $C=C(\lambda, \epsilon, k)$ such that the following conditions hold. Let $\omega=\rho \alpha \rho^{-1} \beta^{-1}$ be a closed path in $\Gamma$ such that $\rho \alpha$ and $\beta \rho^{\prime}$ are paths in $\Gamma$ without backtracking, $\widehat{\alpha}$ and $\widehat{\beta}$ are $(\lambda, \epsilon)$-quasigeodesics in $\widehat{\Gamma}$, and that $\max \left\{l_{\widehat{\Gamma}}(\widehat{\rho}), l_{\widehat{\Gamma}}\left(\widehat{\rho^{\prime}}\right)\right\} \leq k$. If $\eta$ is a component of $\rho \alpha$ isolated in $\omega$, then $d_{\Gamma}\left(\eta_{-}, \eta_{+}\right) \leq C$. In particular, if $\widehat{\alpha}$ and $\widehat{\beta}$ are geodesics in $\widehat{\Gamma}$, then $d_{\Gamma}\left(\eta_{-}, \eta_{+}\right) \leq C(1,0, k)$.

Proof. By Lemma 2.1.4 $\widehat{\rho \alpha}$ and $\widehat{\beta \rho^{\prime}}$ are $\left(\lambda, \epsilon^{\prime}\right)$-quasigeodesics in $\widehat{\Gamma}$, where $\epsilon^{\prime}=\epsilon+(\lambda+1) k$. Note that there exists a closed path $p q^{-1}$ in $\Gamma$ such that $\widehat{p}$ and $\widehat{q}$ are $\left(\lambda, \epsilon^{\prime}\right)$-quasigeodesics without backtracking and without self-intersections in $\widehat{\Gamma}$, and that $\eta$ is a component of $p$ isolated in $p q^{-1}$. It follows that $\widehat{p}$ and $\widehat{q}$ are $\left(\lambda+\epsilon^{\prime}, 0\right)$-quasigeodesics in $\widehat{\Gamma}$. Set $\lambda^{\prime}=\lambda+\epsilon^{\prime}$. The BCP property concludes the first assertion. Since any geodesic is $(1,0)$-quasigeodesic, the second assertion follows.

\subsection{Some properties}

Throughout this section, let $G$ be a group hyperbolic relative to a collection of subgroups $\left\{H_{\lambda}\right\}_{\lambda \in \Lambda}$ in the sense of Osin (see Subsection 2.3.1). We will collect some results done by other people and obtain certain consequences in this section.

Proposition 3.2.1. Osi06c, Proposition 2.36] Let $G$ be a group hyperbolic relative to a collection of subgroups $\left\{H_{\lambda}\right\}_{\lambda \in \Lambda}$. Then the following conditions hold. 
1. For any $g_{1}, g_{2} \in G$, the intersection $g_{1} H_{\lambda} g_{1}^{-1} \cap g_{2} H_{\mu} g_{2}^{-1}$ is finite whenever $\lambda \neq \mu$.

2. For any $\lambda \in \Lambda$ and any $g \notin H_{\lambda}$, the intersection $g H_{\lambda} g^{-1} \cap H_{\lambda}$ is finite.

Let us recall the following definition.

Definition 3.2.2. A subgroup $H$ of a group $\Gamma$ is called malnormal if $H \cap g H g^{-1}$ is trivial for any $g \in \Gamma \backslash H$. We say that $H$ is almost malnormal if $H \cap g H^{-1}$ has finitely many elements for any $g \in \Gamma \backslash H$.

We have immediate consequences as follows.

Corollary 3.2.3. The collection $\left\{H_{\lambda}\right\}_{\lambda \in \Lambda}$ contains exactly one representative of each conjugacy class of infinite parabolic subgroups of $G$.

Proof. It is immediate from the first assertion of Proposition 3.2.1 that infinite subgroups $H_{\lambda}$ and $H_{\mu}$ belong to distinct conjugacy classes, for any $\lambda, \mu \in \Lambda$ and $\lambda \neq \mu$. On the other hand, every parabolic subgroup $P$ of $G$ belongs to the conjugacy class of one of the subgroups in $\left\{H_{\lambda}\right\}_{\lambda \in \Lambda}$. So, each conjugacy class of parabolic subgroups has to have a representative in $\left\{H_{\lambda}\right\}_{\lambda \in \Lambda}$.

Corollary 3.2.4. Every maximal parabolic subgroup $P$ of $G$ is almost malnormal. If $G$ is torsion-free then $P$ is malnormal.

Proof. Let $P=g H_{\lambda} g^{-1}$ for some $g \in G$ and $\lambda \in \Lambda$; let $x \in G \backslash P$. Then $g^{-1} x g \notin H_{\lambda}$. Thus, $g^{-1}\left(P \cap x P x^{-1}\right) g=H_{\lambda} \cap\left(g^{-1} x g\right) H_{\lambda}\left(g^{-1} x g\right)^{-1}$ has finitely many elements because $H_{\lambda}$ is almost malnormal, by the second assertion of Proposition 3.2.1. Hence, $P$ is almost malnormal. The second part of the statement is straightforward.

Corollary 3.2.5. Let $x$ be a parabolic element of infinite order in $G$. Suppose that $[x, y]=x y x^{-1} y^{-1}=1$ for $y \in G$. Then $y$ lies in the same parabolic subgroup as $x$.

Proof. Let $P$ be the parabolic subgroup containing $x$. Note that $P$ is unique; otherwise, it contradicts to the first assertion of Proposition 3.2.1. Assume that $y \notin P$. Note that $x \in y P y^{-1}$ as $[x, y]=1$. Then $x \in P \cap y P y^{-1}$, so that the intersection has infinitely many elements, which is a contradiction to Corollary 3.2.4. Therefore, $y \in P$.

Let us recall that a group is called elementary (or virtually cyclic) if it contains a cyclic subgroup of finite index. The next result is about maximal elementary subgroups of relatively hyperbolic groups.

Theorem 3.2.6. Osi06a, Theorem 4.3, Corollary 1.6 and 1.7] Let $G$ be a group hyperbolic relative to a collection of subgroups $\left\{H_{\lambda}\right\}_{\lambda \in \Lambda}$; and let $g$ be a hyperbolic element of infinite order in $G$. Then the following conditions hold.

1. The element $g$ is contained in a unique maximal elementary subgroup $E(g)$ of $G$, where

$$
E(g)=\left\{f \in G \mid f^{-1} g^{n} f=g^{ \pm n} \text { for some positive integer } n\right\} .
$$

2. The group $G$ is hyperbolic relative to the collection $\left\{H_{\lambda}\right\}_{\lambda \in \Lambda} \cup\{E(g)\}$.

Let us mention some consequences of Theorem 3.2.6. We denote the normalizer of a subgroup $H$ of a group $\Gamma$ by $N_{\Gamma}(H)$, and the centralizer of an element $g$ in $\Gamma$ by $C_{\Gamma}(g)$. 
Corollary 3.2.7. If $P$ is an infinite parabolic subgroup of $G$, then $N_{G}(P)=P$. If $g$ is an infinite order hyperbolic element of $G$ then $N_{G}(E(g))=E(g)$.

Proof. Suppose that $x \in N_{G}(P) \backslash P$. Then the intersection $P \cap x P x^{-1}=P$ is infinite, while $P$ is almost malnormal, by Corollary 3.2.4. The contradiction implies that $N_{G}(P) \subseteq$ $P$. Clearly, $P \subseteq N_{G}(P)$. So, $N_{G}(P)=P$. The second assertion now follows because $G$ is hyperbolic relative to $\left\{H_{\lambda}\right\}_{\lambda \in \Lambda} \cup\{E(g)\}$, by the second assertion of Theorem 3.2.6.

Corollary 3.2.8. Let $g$ be a hyperbolic element of infinite order in $G$. Suppose that $A$ is an infinite subgroup of $E(g)$. Then $N_{G}(A)$ is a subgroup of $N_{G}(E(g))$.

Proof. Assume that $x \in N_{G}(A) \backslash N_{G}(E(g))$. By Corollary 3.2.7, $N_{G}(E(g))=E(g)$; and so $x \notin E(g)$. Thus, the cardinality of $A=x A x^{-1} \cap A \subseteq x E(g) x^{-1} \cap E(g)$ is infinite, which contradicts to $E(g)$ being almost malnormal by Corollary 3.2.4 and the second assertion of Theorem 3.2.6. Therefore, $N_{G}(A) \subseteq N_{G}(E(g))$.

Lemma 3.2.9. Suppose that $G$ is torsion-free and that $g$ is a hyperbolic element of $G$. Then the maximal elementary subgroup $E(g)=\langle a\rangle$ is the maximal cyclic subgroup containing $g$. In particular, the centralizer $C_{G}(g)=\langle a\rangle$ of $g$ is cyclic.

Proof. By the first assertion of Theorem 3.2.6, $E(g)$ is a virtually cyclic group. It is well-known that every torsion-free virtually cyclic group is either trivial or infinite cyclic; for instance, see [Sta68]. Hence, $E(g)$ is the maximal cyclic subgroup containing $g$, that is, $E(g)=\langle a\rangle$ for some $a \in G$. Clearly, $\langle a\rangle \subseteq C_{G}(g)$. On the other hand, it follows from the first assertion of Theorem 3.2 .6 that $C_{G}(g) \subseteq E(g)$; hence, $C_{G}(g) \subseteq\langle a\rangle$. So, $C_{G}(g)=\langle a\rangle$.

Lemma 3.2.10. Let $A$ be an abelian subgroup of $G$. Suppose that $x \in A$ has infinite order. If $x$ is parabolic, then $A$ is contained in a parabolic subgroup; otherwise, $A$ is contained in $E(x)$.

Proof. If $x$ is parabolic, then $x \in P \cap A$ for some parabolic subgroup $P$; Corollary 3.2.5 implies that $A \subseteq P$ as $A$ is abelian. If $x$ is hyperbolic, then the centralizer $C_{G}(x)$ of $x$ is contained in $E(x)$ by the first assertion of Theorem 3.2.6; and note that $A \subseteq C_{G}(x)$. Hence, $A \subseteq E(x)$.

Remark 3.2.11. It is well-known that a hyperbolic group contains no free abelian subgroups of rank 2, i.e., $\mathbb{Z} \times \mathbb{Z}$; for example, see [GS91a, Theorem 5.1]. Torsion-free relatively hyperbolic groups can contain subgroups isomorphic to $\mathbb{Z} \times \mathbb{Z}$ but each one of these has to lie in some parabolic subgroup, by Lemma 3.2.10 and Lemma 3.2.9.

Corollary 3.2.12. Let $G$ be torsion-free, let $H$ be a non-elementary non-parabolic subgroup of $G$, and let $A$ be an abelian subgroup of $H$ which is normal in $H$. Then $A$ is trivial. In particular, every non-elementary non-parabolic subgroup of a torsion-free relatively hyperbolic group has trivial centre.

Proof. Since $A$ is abelian, we have two cases by Lemma 3.2.10. First, $A$ is contained in some parabolic subgroup $P$; and so, $A \subseteq P \cap H$. Since $H \nsubseteq P$, there exists $h \in H \backslash P$ such that $A \subseteq h P h^{-1}$ because $A$ is normal in $H$. Hence, $A \subseteq P \cap h P h^{-1}$, which implies that $A$ is trivial by Corollary 3.2.4. The second case is when $A \subseteq E(x) \cap H$ for some hyperbolic element $x \in G$. Note that $H \nsubseteq E(x)$ and that $E(x)$ is malnormal by Corollary 3.2 .4 and the second assertion of Theorem 3.2.6. A similar argument as in the first case shows that $A$ is trivial. The last assertion follows immediately. 
Given a subgroup $H$ of $G$, we denote by $H^{0}$ the set of all hyperbolic elements of infinite order in $H$.

Lemma 3.2.13. Osi06c, Corollary 4.21] Let $G$ be a finitely generated group hyperbolic relative to a collection of subgroups $\left\{H_{1}, \cdots, H_{m}\right\}$; and let $g \in G^{0}$. If $g^{k}$ is conjugate to $g^{l}$ in $G$ for some nonzero integers $k$ and $l$, then $k= \pm l$.

Definition 3.2.14. Two elements $f$ and $g$ in $G^{0}$ are said to be commensurable in $G$ if $f^{k}$ is conjugate to $g^{l}$ in $G$, for some nonzero integers $k$ and $l$. A subgroup $H$ of $G$ is called suitable, if there exist two non-commensurable elements $f_{1}$ and $f_{2}$ in $H^{0}$ such that $E\left(f_{1}\right) \cap E\left(f_{2}\right)=\{1\}$.

The next two statements are proved in [AMO07.

Proposition 3.2.15. AMO07, Proposition 3.4] Suppose that a group $G$ is hyperbolic relative to a collection of subgroups $\left\{H_{\lambda}\right\}_{\lambda \in \Lambda}$ and that $H$ is a subgroup of $G$. Then $H$ is suitable if and only if $H^{0} \neq \emptyset$ and $E(H)=\bigcap_{g \in H^{0}} E(g)=\{1\}$.

Lemma 3.2.16. AMO07, Lemma 3.3] With the notation of Proposition 3.2.15, if $H$ is a non-elementary subgroup of $G$ and $H^{0} \neq \emptyset$, then $E(H)$ is the maximal finite subgroup of $G$ normalized by $H$.

As an immediate consequence of Proposition 3.2.15 and Lemma 3.2.16 we have the following corollary.

Corollary 3.2.17. Let a group $G$ be torsion-free hyperbolic relative to a collection of subgroups $\left\{H_{\lambda}\right\}_{\lambda \in \Lambda}$. Suppose that $H$ is a non-elementary subgroup of $G$ containing a hyperbolic element $h$. Then $H$ is suitable.

\subsection{Small cancellations}

In this section, our main goal is to introduce Theorem 3.3.4 which is proved by Osin in Osi10]. The proof is based on a certain small cancellation techniques over relatively hyperbolic groups, which generalizes the small cancellation theory over hyperbolic groups developed by Ol'shanskii in Ol'93. We stress that this section is credited to Osin's work of Osi10].

Let $\mathcal{R}$ denote a set words in an alphabet $\mathcal{A}$. We say that $\mathcal{R}$ is symmetrized if for any $R \in \mathcal{R}, \mathcal{R}$ contains all cyclic shifts of $R^{ \pm 1}$.

Definition 3.3.1. Let $G$ be a group generated by a set $\mathcal{A}, \mathcal{R}$ a symmetrized set of words in $\mathcal{A}$. For $\epsilon>0$, a subword $U$ of a word $R \in \mathcal{R}$ is called a $\epsilon$-piece if there exists a word $R^{\prime} \in \mathcal{R}$ such that

(1) $R=U V, R^{\prime}=U^{\prime} V^{\prime}$ for some $V, U^{\prime}, V^{\prime} \in \mathcal{A}^{*}$;

(2) $U^{\prime}=Y U Z$ in $G$ for some $Y, Z \in \mathcal{A}^{*}$ satisfying $\max \{\|Y\|,\|Z\|\} \leq \epsilon$; and

(3) $Y R Y^{-1} \neq R^{\prime}$ in $G$.

Similarly, a subword $U$ of $R \in \mathcal{R}$ is called a $\epsilon^{\prime}$-piece if

(1') $R=U V U^{\prime} V^{\prime}$ for some $V, U^{\prime}, V^{\prime} \in \mathcal{A}^{*}$; and 

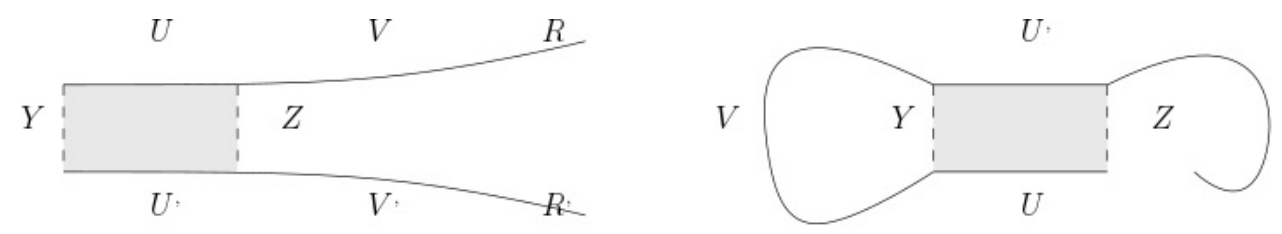

Figure 3.2: $\epsilon$-piece and $\epsilon^{\prime}$-piece

(2') $U^{\prime}=Y U^{ \pm 1} Z$ in $G$ for some $Y, Z$ satisfying $\max \{\|Y\|,\|Z\|\} \leq \epsilon$.

See Figure 3.2 for Definition 3.3.1.

Given a group $G$ generated by a set $\mathcal{A}$, a word $R$ in $\mathcal{A}$ is said to be geodesic (respectively $(\lambda, c)$-quasigeodesic), if any path in the Cayley graph $\Gamma(G, \mathcal{A})$ labeled by $R$ is geodesic (respectively $(\lambda, c)$-quasigeodesic).

Definition 3.3.2. We say that the set $\mathcal{R}$ satisfies the $C(\epsilon, \mu, \lambda, c, \rho)$ small cancellation condition for some $\epsilon \geq 0, \mu>0, \lambda>0, c \geq 0, \rho>0$, if

(1) $\|R\| \geq \rho$ for any $R \in \mathcal{R}$;

(2) any word $R \in \mathcal{R}$ is $(\lambda, c)$-quasigeodesic;

(3) for any $\epsilon$-piece of any word $R \in \mathcal{R}$, the inequality

$$
\max \left\{\|U\|,\left\|U^{\prime}\right\|\right\} \leq \mu\|R\|
$$

holds (using the notation of Definition 3.3.1).

Further the set $\mathcal{R}$ satisfies the $C_{1}(\epsilon, \mu, \lambda, c, \rho)$ small cancellation condition if in addition the condition (3) holds for any $\epsilon^{\prime}$-piece for any word $R \in \mathcal{R}$.

In what follows, let $G$ be a group hyperbolic relative to a collection of subgroups $\left\{H_{\lambda}\right\}_{\lambda \in \Lambda}$; let $X$ be a relative generating set for $G$ with respect to $\left\{H_{\lambda}\right\}_{\lambda \in \Lambda}$; and let $\mathcal{H}=\bigsqcup_{\lambda \in \Lambda}\left(\widetilde{H}_{\lambda} \backslash\{1\}\right)$ be defined in 2.2 . We set $\mathcal{A}=X \cup \mathcal{H}$.

The following statement shows that a certain set of word over the alphabet $\mathcal{A}$ satisfies the small cancellation conditions.

Theorem 3.3.3. Osi10, Theorem 7.5] Suppose that $W=x a_{1} b_{1} \cdots a_{n} b_{n}$ for some $n \geq 1$ is a word in $\mathcal{A}^{*}$ satisfying the following conditions:

- $x \in X \cup\{1\}$;

- $a_{1}, \cdots, a_{n}$ and $b_{1}, \cdots, b_{n}$ are elements of $H_{\alpha}$ and $H_{\beta}$, respectively, where $H_{\alpha} \cap H_{\beta}=$ $\{1\}$

- the elements $a_{1}, \cdots, a_{n}, b_{1}, \cdots, b_{n}$ are geodesics and each of the length is at least a constant $K(\epsilon)$ with respect to a finite subset of $G$; and

- $a_{i} \neq a_{j}^{ \pm 1}, b_{i} \neq b_{j}^{ \pm 1}$ whenever $i \neq j$ and $a_{i} \neq a_{i}^{-1}, b_{i} \neq b_{i}^{-1}$, for all $i, j \in\{1, \cdots, n\}$.

Then the set of all cyclic shifts of $W^{ \pm 1}$ satisfies the $C_{1}\left(\epsilon, \frac{3 \epsilon+11}{n}, \frac{1}{3}, 2,2 n+1\right)$ small cancellation condition.

Now we are ready to provide the main result of this section. 
Theorem 3.3.4. Osi10, Theorem 2.4, Lemma 5.1] Let $G$ be a group hyperbolic relative to a collection of subgroups $\left\{H_{\lambda}\right\}_{\lambda \in \Lambda}, H$ a suitable subgroup of $G$, and $t_{1}, \cdots, t_{m}$ arbitrary elements of $G$. Then there exists an epimorphism $\eta: G \rightarrow \bar{G}$ such that:

1. The group $\bar{G}$ is hyperbolic relative to $\left\{\eta\left(H_{\lambda}\right)\right\}_{\lambda \in \Lambda}$.

2. For any $i=1, \cdots, m$, we have $\eta\left(t_{i}\right) \in \eta(H)$.

3. The restriction of $\eta$ to $\bigcup_{\lambda \in \Lambda} H_{\lambda}$ is injective.

4. $\eta(H)$ is a suitable subgroup of $\bar{G}$.

5. Every element of finite order in $\bar{G}$ is an image of an element of finite order in $G$. In particular, if all hyperbolic elements of $G$ have infinite order, then all hyperbolic elements of $\bar{G}$ have infinite order.

6. For any $N>0$, the restriction of $\eta$ to the subset of elements of length at most $N$ with respect to $X \cup \mathcal{H}$ is injective, where $X$ is a relative generating set for $G$ with respect to $\left\{H_{\lambda}\right\}_{\lambda \in \Lambda}$ and $\mathcal{H}$ defined in (2.2).

Proof. We give a very rough idea of the proof here. Let us consider the case $m=$ 1. Since $H$ is suitable, there are non-commensurable elements $h_{1}, h_{2} \in H^{0}$ such that $E\left(h_{1}\right) \cap E\left(h_{2}\right)=\{1\}$. By the second assertion of Theorem 3.2.6, $G$ is hyperbolic relative to $\left\{H_{\lambda \in \Lambda}\right\}_{\lambda \in \Lambda} \cup\left\{E\left(h_{1}\right)\right\} \cup\left\{E\left(h_{2}\right)\right\}$. By Theorem 3.3.3, there are $n$ and $m_{1}, \cdots, m_{n}$ such that the set $\mathcal{R}$ of all cyclic shifts and their inverses of the word

$$
R=t_{1} h_{1}^{m_{1}} h_{2}^{m_{2}} \cdots h_{1}^{m_{n}} h_{2}^{m_{n}}
$$

in the alphabet $X \cup \mathcal{H} \cup\left(E\left(h_{1}\right) \backslash\{1\}\right) \cup\left(E\left(h_{2}\right) \backslash\{1\}\right)$ satisfies a certain small cancellation condition. Let $\bar{G}$ be the quotient of $G$ obtained by imposing the relation $R=1$; and let $\eta: G \rightarrow \bar{G}$ be the corresponding natural homomorphism. By using technical results on the small cancellation condition proved in Osi10], the above assertions follow. Now the theorem follows if we apply the case for $m=1$ several times.

\subsection{Combination theorems}

In this section, we will present the combination theorems for relatively hyperbolic groups proved by Osin in Osi06b]. The results were also proved by Alibegovic in Ali05] and Dahmani in Dah03] and used to show that limit groups introduced by Sela Sel01 are hyperbolic relative to collections of maximal noncyclic abelian subgroups. We begin with the following definitions.

Definition 3.4.1. Let $A, B$ and $C$ be groups; and let $\alpha_{1}: C \rightarrow A$ and $\alpha_{2}: C \rightarrow B$ be monomorphisms. If $\langle X \mid \mathcal{R}\rangle$ and $\langle Y \mid \mathcal{S}\rangle$ are presentations of $A$ and $B$, respectively, we define the amalgamated product $A *_{C} B$ by the presentation

$$
\left\langle X, Y \mid \mathcal{R}, \mathcal{S}, \alpha_{1}(c)=\alpha_{2}(c) \forall c \in C\right\rangle .
$$

If $C$ is the trivial group, then $A *_{C} B$ is denoted by $A * B$ and is called the free product of $A$ and $B$. 
Definition 3.4.2. Let $A$ and $C$ be groups; and let $\alpha_{1}: C \rightarrow A$ and $\alpha_{2}: C \rightarrow A$ be monomorphisms. If $\langle X \mid \mathcal{R}\rangle$ is a presentation of $A$ and $t \notin X$, we define the $H N N$ extension $A *_{C}$ by the presentation

$$
\left\langle X, t \mid \mathcal{R}, t^{-1} \alpha_{1}(c) t=\alpha_{2}(c) \forall c \in C\right\rangle .
$$

Theorem 3.4.3. Osi06b, Corollary 1.4] Suppose that a group $H$ is hyperbolic relative to $\left\{H_{\lambda}\right\}_{\lambda \in \Lambda} \cup\{K\}$. Assume also that $K$ is finitely generated and for some $\lambda \in \Lambda$, there is a monomorphism $\iota: K \rightarrow H_{\lambda}$. Then the HNN extension

$$
G=\left\langle H, t \mid t^{-1} k t=\iota(k) \forall k \in K\right\rangle
$$

is hyperbolic relative to $\left\{H_{\lambda}\right\}_{\lambda \in \Lambda}$.

Proof. We give a rough idea of the proof. Since $H$ is hyperbolic relative to $\left\{H_{\lambda}\right\}_{\lambda \in \Lambda} \cup\{K\}$. Then $H$ has a finite relative presentation with respect to $\left\{H_{\lambda}\right\}_{\lambda \in \Lambda} \cup\{K\}$, i.e.,

$$
H=\left\langle X, K, H_{\lambda}, \lambda \in \Lambda \mid \mathcal{R}\right\rangle .
$$

It follows that the HNN extension $G=\left\langle H, t \mid t^{-1} k t=\iota(k) \forall k \in K\right\rangle$ has a finite relative presentation

$$
G=\left\langle X \cup Y \cup\{t\}, H_{\lambda}, \lambda \in \Lambda \mid \mathcal{R}^{\prime} \cup \mathcal{T}\right\rangle
$$

where

- $Y$ is an arbitrary symmetrized finite generating set of $K$,

- $\mathcal{R}^{\prime}$ is a finite set words obtained from $R \in \mathcal{R}$ by replacing each $k \in K$ with a shortest word in $Y$ representing the element $k$ in $H$, and

- $\mathcal{T}=\left\{t^{-1} y t=\iota(y) \forall y \in Y\right\}$.

Let $\mathcal{Z}=X \cup Y \cup\{t\} \cup \mathcal{H}$. It remains to show that there is a constant $K>0$ such that for any word $\mathrm{w}$ in $\mathcal{Z}^{*}$, representing the identity of $G, \operatorname{Area}^{\text {rel }}(\mathrm{w}) \leq K\left\|_{\mathrm{w}}\right\|_{\mathcal{Z}}$, where $\mathcal{H}$ is defined in $(2.2)$. This follows from a technical result developed from van Kampen diagrams over HNN extensions.

The following theorem is a consequence of Theorem 3.4.3.

Theorem 3.4.4. Osi06b, Corollary 1.5] Suppose that $A$ and $B$ are groups, hyperbolic relative to $\left\{A_{\mu}\right\}_{\mu \in M} \cup\{K\}$ and $\left\{B_{\nu}\right\}_{\nu \in N}$, respectively. Assume in addition that $K$ is finitely generated and for some $\eta \in N$, there is a monomorphism $\xi: K \rightarrow B_{\eta}$. Then the amalgamated product $A *_{K=\xi(K)} B$ is hyperbolic relative to $\left\{A_{\mu}\right\}_{\mu \in M} \cup\left\{B_{\nu}\right\}_{\nu \in N}$. 


\section{Chapter 4}

\section{Some algorithmic problems on relatively hyperbolic groups}

In this chapter, we will provide two algorithms solving the word and conjugacy problems in relatively hyperbolic groups, and we will discuss the time complexity of each problem.

Throughout this chapter, a group is called relatively hyperbolic if it is a relatively hyperbolic group in the sense of Farb and satisfies the BCP property (see subsection 2.3.2. In this chapter, we accept the following notation and technical agreements.

\section{Convention 4.0.1.}

- $G$ is a group hyperbolic relative to the set of subgroups $\mathcal{P}=\left\{P_{1}, \cdots, P_{m}\right\}$, defined by a finite relative presentation

$$
\left\langle S_{0}, P_{1}, \cdots, P_{m} \mid R=1, R \in \mathcal{R}\right\rangle,
$$

and given finite generating sets $S_{1}, \cdots, S_{m}$ such that $P_{i}=\left\langle S_{i}\right\rangle$ for all $1 \leq i \leq m$.

- Let $S=S_{0} \sqcup S_{1} \sqcup \cdots \sqcup S_{m}$ and let $\widehat{S}=S_{0} \cup P_{1} \cup \cdots \cup P_{m}$. Let $\Gamma$ be the Cayley graph of $G$ with respect to $S$; let $\widehat{\Gamma}$ be the coned-off Cayley graph of $G$ with respect to $\mathcal{P}$ (or $\widehat{S}$ ); and let $\Gamma_{i}$ be the Cayley graph of $P_{i}$ with respect to $S_{i}$ for all $1 \leq i \leq m$. Let $\mathcal{A}=S^{ \pm 1}$ and $\mathcal{A}_{i}=S_{i}^{ \pm 1}$ for all $1 \leq i \leq m$. Let $C(\lambda, \epsilon, k)$ be the constant from Lemma 3.1.7.

- For technical reasons, we assume that the hyperbolicity $\delta \geq 1$ for $\widehat{\Gamma}$, and that $s_{0} w \notin$ $\mathcal{R}$ for any $s_{0} \in S_{0}$ and $w \in \mathcal{A}_{i}^{*}$ for all $1 \leq i \leq m$.

\subsection{The word problem}

\subsubsection{Estimates}

We establish some estimates in this subsection which will be used for solving the word problem of $G$.

Definition 4.1.1. Let $\mathcal{C}$ be a set of paths in $\widehat{\Gamma}$ satisfying some properties, e.g., the set $\mathcal{C}(\lambda, \epsilon)$ of all $(\lambda, \epsilon)$-quasigeodesics in $\widehat{\Gamma}$ for some $\lambda \geq 1$ and $\epsilon \geq 0$, or the set $\mathcal{C}(L)$ of all $L$-local geodesics in $\widehat{\Gamma}$ for some $L>1$. We say that path $\omega$ is an alternate $\mathcal{C}$-path in $\Gamma$ if

$$
\omega=\alpha_{1} \circ \eta_{1} \circ \alpha_{2} \circ \eta_{2} \circ \cdots \circ \alpha_{n} \circ \eta_{n},
$$


where $\alpha_{i}$ is a path in $\Gamma$ such that $\widehat{\alpha}_{i} \in \mathcal{C}, \eta_{i}$ is a component of $\omega$ in $\Gamma$ for all $1 \leq i \leq n$, and $\alpha_{j}, \eta_{k}$ are non-empty for all $2 \leq j \leq n$ and $1 \leq k \leq n-1$.

Let $\alpha$ and $\beta$ be paths in $\Gamma$; and let $\omega=\alpha \beta$ be a concatenation of $\alpha$ and $\beta$ such that $\alpha_{+}=\beta_{-}$. Suppose that $\alpha=\alpha_{1} \alpha_{2}, \beta=\beta_{1} \beta_{2}$ and $\alpha_{2} \beta_{1}$ is a component of $\omega$. Then $\omega=\alpha^{\prime} \beta^{\prime}$ is a concatenation of $\alpha^{\prime}=\alpha_{1} \alpha_{2} \beta_{1}$ and $\beta^{\prime}=\beta_{2}$. In this case, we denote by $\omega=\alpha^{\prime} \circ \beta^{\prime}$ and we use the notation $\circ$ to emphasize this separation throughout the thesis.

Lemma 4.1.2. Let $\alpha \beta^{-1}$ be a closed path in $\Gamma$, where $\alpha=\alpha_{1} \circ \eta_{1} \circ \cdots \circ \alpha_{n} \circ \eta_{n}$ is an alternate $\mathcal{C}(\lambda, \epsilon)$-path in $\Gamma$ without backtracking, and $\beta$ is a path in $\Gamma$ without backtracking such that $\widehat{\beta} \in \mathcal{C}(\lambda, \epsilon)$. If for all $1 \leq i \leq n, \eta_{i}$ is connected to a component $\sigma_{i}$ of $\beta$, then there are two vertices $u$ and $v$ in $\left\{\sigma_{i_{-}}, \sigma_{i_{+}}\right\}$such that $\max \left\{d_{\Gamma}\left(\eta_{i_{-}}, u\right), d_{\Gamma}\left(\eta_{i_{+}}, v\right)\right\} \leq C(\lambda, \epsilon, 1)$.

Proof. We will show that there is a vertex $v \in\left\{\sigma_{i_{-}}, \sigma_{i_{+}}\right\}$such that $d_{\Gamma}\left(\eta_{i_{+}}, v\right) \leq C(\lambda, \epsilon, 1)$ for all $1 \leq i \leq n$. Let $\eta_{n}$ be non-empty; and let $i \leq n-1$. Then there are two possibilities:

(1) $\sigma_{i+1_{-}} \in \beta_{\sigma_{i_{+}, \beta_{+}}}$, or

(2) $\sigma_{i+1+} \in \beta_{\beta_{-}, \sigma_{i_{-}}}$.

If (1) holds, we denote by $\zeta_{1}$ and $\tau_{1}$ components connecting $\sigma_{i+}, \eta_{i_{+}}$and $\eta_{i+1-}, \sigma_{i+1-}$, respectively. If (2) holds, we denote by $\zeta_{2}$ and $\tau_{2}$ components connecting $\sigma_{i-}, \eta_{i+}$ and $\eta_{i+1_{-}}, \sigma_{i+1_{+}}$. Since $\eta_{i}$ is connected to $\sigma_{i}$ for all $1 \leq i \leq n$ and $\alpha, \beta$ are without backtracking, $\alpha_{i+1} \circ \tau_{1}$ and $\beta_{\sigma_{i+1}, \sigma_{i+}} \circ \zeta_{1}$ are paths without backtracking, and $\zeta_{1}$ is isolated in the closed path $\rho_{1}=\alpha_{i+1} \circ \tau_{1} \circ \beta_{\sigma_{i+1_{-}}, \sigma_{i+}} \circ \zeta_{1}$. Similarly, $\alpha_{i+1} \circ \tau_{2}$ and $\beta_{\sigma_{i+1_{+}}, \sigma_{i_{-}}} \circ \zeta_{2}$ are paths without backtracking, and $\zeta_{2}$ is isolated in the closed path $\rho_{2}=\alpha_{i+1} \circ \tau_{2} \circ \beta_{\sigma_{i+1+}, \sigma_{i-}} \circ \zeta_{2}$. See Figure 4.1. Note that $\widehat{\alpha_{i+1}}$ and any subpath of $\widehat{\beta}$ are $(\lambda, \epsilon)$-quasigeodesics in $\widehat{\Gamma}$. By
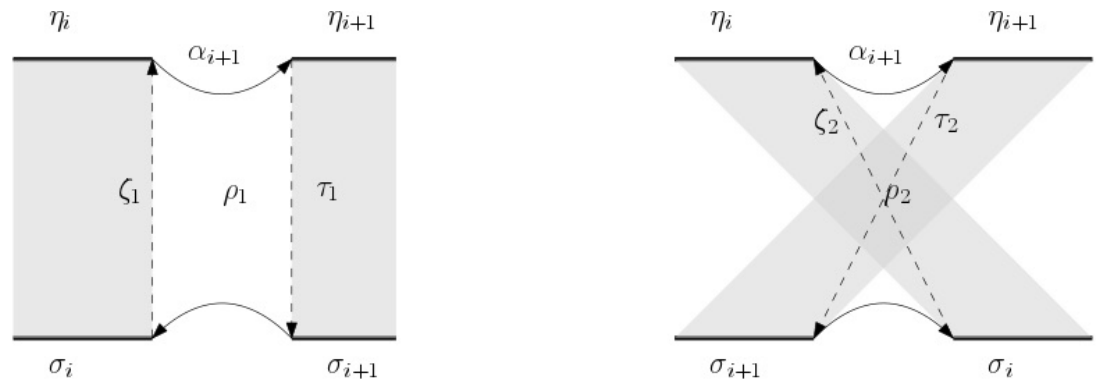

Figure 4.1: Two cases for connected components

Lemma 3.1.7, $d_{\Gamma}\left(\zeta_{1_{-}}, \zeta_{1_{+}}\right) \leq C(\lambda, \epsilon, 1)$ or $d_{\Gamma}\left(\zeta_{2_{-}}, \zeta_{2+}\right) \leq C(\lambda, \epsilon, 1)$. Therefore, we have $d_{\Gamma}\left(\eta_{i_{+}}, v\right) \leq C(\lambda, \epsilon, 1)$, where $v \in\left\{\sigma_{i_{-}}, \sigma_{i+}\right\}$.

If $i=n-1$ and $\eta_{n}$ is empty, then it is similar as the above case (1) to obtain $d_{\Gamma}\left(\eta_{i_{+}}, \sigma_{i+}\right) \leq C(\lambda, \epsilon, 1)$. If $i=n$ and $\eta_{n}$ is non-empty, then $\eta_{n_{+}}=\sigma_{n+}$ because $\eta_{n}$ is connected to $\sigma_{n}$ and $\alpha, \beta$ are without backtracking; it follows that $d_{\Gamma}\left(\eta_{n_{+}}, \sigma_{n_{+}}\right)=0<$ $C(\lambda, \epsilon, 1)$. Therefore, there is a vertex $v \in\left\{\sigma_{i_{-}}, \sigma_{i_{+}}\right\}$such that $d_{\Gamma}\left(\eta_{i_{+}}, v\right) \leq C(\lambda, \epsilon, 1)$ for all $1 \leq i \leq n$.

The other assertion, there is a vertex $u \in\left\{\sigma_{i_{-}}, \sigma_{i+}\right\}$ such that $d_{\Gamma}\left(\eta_{i_{-}}, u\right) \leq C(\lambda, \epsilon, 1)$ can be proven similarly. All together, the statement is proven.

It is straightforward to obtain the following result. 
Corollary 4.1.3. With the notation and assumptions of Lemma 4.1.2, let $d_{\Gamma}\left(\sigma_{i_{-}}, \sigma_{i_{+}}\right) \leq$ $k$ for some constant $k$. Then $d_{\Gamma}\left(\eta_{i_{-}}, \eta_{i_{+}}\right) \leq 2 C(\lambda, \epsilon, 1)+k$.

Proposition 4.1.4. Let $\omega=\alpha_{1} \circ \eta_{1} \circ \cdots \circ \alpha_{n} \circ \eta_{n}$ be an alternate $\mathcal{C}(\lambda, \epsilon)$-path in $\Gamma$. Suppose the following conditions hold:

1. $\omega$ is without backtracking; and

2. there exists a geodesic $\widehat{\omega_{1}}$ in $\widehat{\Gamma}$ connecting the endpoints of $\widehat{\omega}$ such that a component of $\omega$ from $\left\{\eta_{1}, \cdots, \eta_{n}\right\}$ is isolated in $\omega \omega_{1}^{-1}$.

Then there exists $t \in\{1, \cdots, n\}$ such that $d_{\Gamma}\left(\eta_{t_{-}}, \eta_{t_{+}}\right) \leq k(\lambda, \epsilon)$, where

$$
k(\lambda, \epsilon)=2 C(\lambda, \epsilon, 1)+C(1,0,1) .
$$

Proof. Firstly, if $\alpha_{1}$ is non-empty (or $\alpha_{1}$ is empty), and if there is a geodesic $\widehat{\gamma}$ in $\widehat{\Gamma}$ connecting $\widehat{\omega}_{-}$and $\widehat{\eta}_{1_{+}}$(or $\widehat{\omega}_{-}$and ${\widehat{\alpha_{2}}}_{+}$, respectively) such that $\eta_{1}$ is isolated in the closed path $\alpha_{1} \circ \eta_{1} \circ \gamma^{-1}$ (or $\eta_{1} \circ \alpha_{2} \circ \gamma^{-1}$, respectively) in $\Gamma$, then $d_{\Gamma}\left(\eta_{1_{-}}, \eta_{1_{+}}\right) \leq C(\lambda, \epsilon, 1)$ by Lemma 3.1 .7 .

Next, suppose that $\alpha_{1}$ is non-empty (or $\alpha_{1}$ is empty), and that every path $\gamma^{\prime}$ in $\Gamma$ connecting $\omega_{-}$and $\eta_{1_{+}}$(or $\omega_{-}$and $\alpha_{2+}$, respectively) such that $\widehat{\gamma^{\prime}}$ is a geodesic in $\widehat{\Gamma}$ has a component connected to $\eta_{1}$. By tracking $\widehat{\omega}$ from ${\widehat{\gamma^{\prime}}}_{+}$, the second assumption implies that there exists a geodesic $\widehat{\gamma}$ in $\widehat{\Gamma}$ with the following properties:

$(\gamma 1) \widehat{\gamma}_{-}=\widehat{\omega}_{-}, \widehat{\gamma}_{+}=u \in \widehat{\alpha}_{j} \circ \widehat{\eta}_{j}$ for some $j \in\{2, \cdots, n\}$ if $\alpha_{1}$ is non-empty (or $j \in$ $\{3, \cdots, n\}$ if $\alpha_{1}$ is empty);

$(\gamma 2)$ there exists a component $\eta_{t}$ of $\omega_{\omega_{-}, u}$ from $\left\{\eta_{1}, \cdots, \eta_{n}\right\}$ isolated in $\omega_{\omega_{-}, u} \gamma^{-1}$;

$(\gamma 3)$ among all of the paths that satisfy $(\gamma 1)$ and $(\gamma 2), \widehat{\gamma}$ is such that $j$ is minimal; and

$(\gamma 4)$ among all of the paths that satisfy $(\gamma 1),(\gamma 2)$ and $(\gamma 3), \widehat{\gamma}$ is such that $l_{\widehat{\Gamma}}\left(\widehat{\omega}_{\widehat{\omega}_{-}, u}\right)$ is minimal.

The existence of $\widehat{\gamma}$ implies that there exists a geodesic $\widehat{\gamma^{\prime}}$ in $\widehat{\Gamma}$ with the following properties:

$\left(\gamma^{\prime} 1\right){\widehat{\gamma^{\prime}}}_{-}=\widehat{\omega}_{-}$and ${\widehat{\gamma^{\prime}}}_{+}^{\prime}=v \in \widehat{\omega}_{\widehat{\omega}_{-}, u} ;$

$\left(\gamma^{\prime} 2\right) d_{\widehat{\Gamma}}(v, u)=1 ;$ and

$\left(\gamma^{\prime} 3\right)$ every component of $\omega_{\omega_{-}, v}$ from $\left\{\eta_{1}, \cdots, \eta_{n}\right\}$ is connected to a component of $\gamma^{\prime}$.

By $(\gamma 2)$, there is a component $\eta_{t}$ of $\omega_{\omega_{-}, u}$ from $\left\{\eta_{1}, \cdots, \eta_{n}\right\}$ such that $\eta_{t}$ is not connected to a component of $\gamma$. Note that $\omega_{\omega_{-}, u}$ is a concatenation of $\omega_{\omega_{-}, v}$ and $\omega_{v, u}$. If $\eta_{t}$ is lying on $\omega_{\omega_{-}, v}$, then $\eta_{t}$ is connected to a component of $\gamma^{\prime}$ by $\left(\gamma^{\prime} 3\right)$. If $\eta_{t}$ is lying on $\omega_{v, u}$, then $\eta_{t}$ may or may not be connected to a component of $\gamma^{\prime}$. It follows that there are two cases to discuss:

(1) $\eta_{t} \neq \omega_{v, u}$; or

(2) $\eta_{t}=\omega_{v, u}$ 

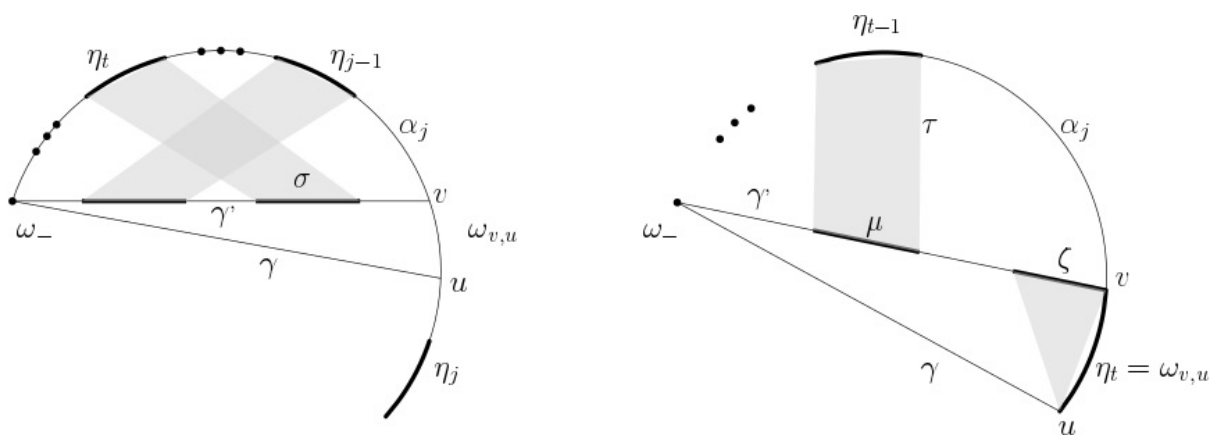

Figure 4.2: Two cases for $\eta_{t}$

If (1) holds, then $\eta_{t}$ is connected to a component $\sigma$ of $\gamma^{\prime}$ by $\left(\gamma^{\prime} 3\right)$. See the left of Figure 4.2. If $\omega_{v, u}$ is a component of $\omega$, then $\sigma$ is not connected to $\omega_{v, u}$; otherwise, $\eta_{t}$ is connected to $\omega_{v, u}$, which contradicts to the first assumption. It follows that $\sigma$ is isolated in the closed path $\gamma^{\prime} \omega_{v, u} \gamma^{-1}$ in $\Gamma$. By Lemma 3.1.7, $d_{\Gamma}\left(\sigma_{-}, \sigma_{+}\right) \leq C(1,0,1)$. By Corollary 4.1.3. $d_{\Gamma}\left(\eta_{t_{-}}, \eta_{t_{+}}\right) \leq 2 C(\lambda, \epsilon, 1)+C(1,0,1)$.

If $(2)$ holds, then $\eta_{t}$ may or may not be connected to a component of $\gamma^{\prime}$. First, suppose that $\eta_{t}$ is connected to a component $\zeta$ of $\gamma^{\prime}$. Note that $\widehat{\gamma^{\prime}}$ is a path in $\widehat{\Gamma}$ without backtracking by Lemma 3.1.6. Then $\zeta_{+}=\eta_{t_{-}}$. See the right of Figure 4.2. Note that $\zeta$ is isolated in the closed path $\alpha_{j} \circ \gamma_{v, \mu_{+}}^{\prime} \circ \tau$ in $\Gamma$, where $\mu$ is a component of $\gamma^{\prime}$ connected to $\eta_{t-1}$ and $\tau$ is a component connecting $\mu_{+}$and $\eta_{t-1+}$. Lemma 3.1.7 implies that $d_{\Gamma}\left(\zeta_{-}, \zeta_{+}\right) \leq C(\lambda, \epsilon, 1)$. It follows that $\zeta \eta_{t}$ is a component isolated in the closed path $\gamma^{\prime} \eta_{t} \gamma^{-1}$ in $\Gamma$. So, $d_{\Gamma}\left(\zeta_{-}, \eta_{t_{+}}\right) \leq C(1,0,1)$ by Lemma 3.1.7. Hence, $d_{\Gamma}\left(\eta_{t_{-}}, \eta_{t_{+}}\right) \leq$ $d_{\Gamma}\left(\zeta_{-}, \zeta_{+}\right)+d_{\Gamma}\left(\zeta_{-}, \eta_{t+}\right) \leq C(\lambda, \epsilon, 1)+C(1,0,1)$. Now, if $\eta_{t}$ is not connected to any component of $\gamma^{\prime}$, then $\eta_{t}$ is isolated in the closed path $\gamma^{\prime} \circ \eta_{t} \circ \gamma^{-1}$; and so, Lemma 3.1.7 implies that $d_{\Gamma}\left(\eta_{t_{-}}, \eta_{t_{+}}\right) \leq C(1,0,1)$.

All together, $d_{\Gamma}\left(\eta_{t_{-}}, \eta_{t_{+}}\right) \leq 2 C(\lambda, \epsilon, 1)+C(1,0,1)$; and therefore, the statement is proven.

Theorem 4.1.5. Let $k(3,2 \delta)$ be the constant from Proposition 4.1.4; and let $L=8 \delta+1$. If $\omega=\alpha_{1} \circ \eta_{1} \circ \cdots \circ \alpha_{n} \circ \eta_{n}$ is an alternate $\mathcal{C}(L)$-path in $\Gamma$ such that $d_{\Gamma}\left(\eta_{i_{-}}, \eta_{i_{+}}\right)>k(3,2 \delta)$ for all $1 \leq i \leq n$, then $\omega$ has the following properties:

1. $\omega$ is a path without backtracking;

2. for any geodesic $\widehat{\gamma}$ in $\widehat{\Gamma}$ connecting the endpoints of $\omega$, each $\eta_{i}$ is connected to a component of $\gamma$ in $\Gamma$; and

3. $\omega_{-} \neq \omega_{+}$.

Proof. We claim that $\alpha_{i} \circ \eta_{i}$ and $\eta_{j} \circ \alpha_{j+1}$ are paths without backtracking for all $1 \leq i \leq n$, $1 \leq j \leq n-1$. Suppose that $\alpha_{i} \circ \eta_{i}$ has a backtracking for some $1 \leq i \leq n$. Note that $\alpha_{i}$ is a path without backtracking by Lemma 3.1.6. It follows that there exists a subpath $\alpha$ of $\alpha_{i}$ such that $\alpha_{+}=\alpha_{i+}, \phi(\alpha) \notin \mathcal{A}_{r}^{*}$ and $\phi\left(\left[\alpha_{-}, \alpha_{+}\right]\right) \in \mathcal{A}_{r}^{*}$ for some $1 \leq r \leq m$. By Corollary 2.2.6, $\widehat{\alpha}$ is a $(3,2 \delta)$-quasigeodesic in $\widehat{\Gamma}$; and so, $l_{\widehat{\Gamma}}(\widehat{\alpha}) \leq 3+2 \delta<L$. Since $\widehat{\alpha} \in \mathcal{C}(L)$, we have $l_{\widehat{\Gamma}}(\widehat{\alpha})=1$. Hence, $\phi(\alpha) \in S_{0}$, which contradicts to our assumption in Convention 4.0.1 that $\mathcal{R}$ does not contain $\phi(\alpha) \phi\left(\left[\alpha_{+}, \alpha_{-}\right]\right)$. Therefore, the claim is proven.

Suppose that $\omega$ has a backtracking. Then there exists a subpath $\omega^{\prime}$ of $\omega$ such that $\left[\omega^{\prime}, \omega_{+}^{\prime}\right]$ is an isolated $P_{s^{-}}$component of the closed path $\omega^{\prime} \circ\left[\omega_{-}^{\prime}, \omega_{+}^{\prime}\right]^{-1}$ in $\Gamma$ for some 
$P_{s} \in \mathcal{P}$, and that $\phi\left(\omega^{\prime}\right) \notin \mathcal{A}_{s}^{*}$. We can assume that $\omega^{\prime}$ is a path without backtracking; otherwise, we choose a backtracking on $\omega^{\prime}$ which has shorter length in $\Gamma$ than $l_{\Gamma}\left(\omega^{\prime}\right)$. It follows that $\omega^{\prime}$ is not a subpath of $\alpha_{i}$ for some $1 \leq i \leq n$; otherwise, it contradicts to that $\alpha_{i} \circ \eta_{i}$ and $\eta_{j} \circ \alpha_{j+1}$ are paths without backtracking for all $1 \leq i \leq n, 1 \leq j \leq n-1$. Thus, $\omega^{\prime}$ contains $\alpha_{i}^{\prime} \circ \eta_{i} \circ \alpha_{i+1}^{\prime}$ for some $1 \leq i \leq n-1$, where $\alpha_{i}^{\prime}$ and $\alpha_{i+1}^{\prime}$ are non-empty subpaths of $\alpha_{i}$ and $\alpha_{i+1}$, respectively. By Corollary 2.2.6, $\widehat{\alpha_{i}}$ is a $(3,2 \delta)$-quasigeodesic in $\widehat{\Gamma}$ for all $1 \leq i \leq n$. Applying Proposition 4.1.4 to $\omega^{\prime}$, there exists a component $\eta_{t}$ of $\omega^{\prime}$ from $\left\{\eta_{1}, \cdots, \eta_{n}\right\}$ such that $d_{\Gamma}\left(\eta_{t-}, \eta_{t+}\right) \leq k(3,2 \delta)$. However, this is a contradiction to our assumption that $d_{\Gamma}\left(\eta_{i_{-}}, \eta_{i_{+}}\right)>k(3,2 \delta)$ for all $1 \leq i \leq n$. Hence, $\omega$ is a path in $\Gamma$ without backtracking; and so, the first assertion is proven. The second assertion follows from Proposition 4.1.4.

Suppose that $\omega$ is a closed path in $\Gamma$. The first and second assertions imply that $\omega=\alpha_{1} \circ \eta_{1}$ or $\eta_{1} \circ \alpha_{2}$. By the same argument as above paragraph, $\phi\left(\alpha_{k}\right) \in S_{0}$ for all $k \in\{1,2\}$. It contradicts to the property of $\mathcal{R}$ in Convention 4.0.1, that is, $s_{0} w \notin \mathcal{R}$ for any $s_{0} \in S_{0}$ and $w \in \mathcal{A}_{i}^{*}$ for all $1 \leq i \leq m$. Therefore, the last assertion is proven.

Remark 4.1.6. Theorem 4.1.5 generalizes a result proved by Martínez-Pedroza in [MP09, Proposition 3.1]. Martínez-Pedroza's result is obtained from an alternate $\mathcal{C}(G e o)$-path in $\Gamma$ where $\mathcal{C}(G e o)$ is the set of all geodesics in $\widehat{\Gamma}$.

\subsubsection{The algorithm}

We establish an algorithm in this subsection which solves the word problem of relatively hyperbolic groups, whenever a solution to the word problem in each parabolic subgroup is given.

Before introducing our algorithm, let us recall the Dehn's algorithm for solving the word problem of any hyperbolic group in linear time from Algorithm DA in Section 7.1 of [BH05] or pages 33-35 in $\left[\mathrm{ABC}^{+} 91\right.$ ].

Algorithm 4.1.7. BH05, Algorithm DA] Let $L \geq 8 \delta+1$. Suppose that $H$ is a hyperbolic group defined by a Dehn presentation $H=\langle Z \mid T=1, T \in \mathcal{T}\rangle$. Given a freely reduced word $\mathrm{w} \in\left(Z^{ \pm}\right)^{*}$, one looks for subwords $u$ such that $u v=T,\|u\| \leq L$ and $\|u\|>\|v\|$; if there is no such subword, one stops and outputs that w does not represent the identity of $H$; if such a word $u$ is found, it is replaced by $v^{-1}$. The algorithm then repeats the search for subwords of the (edited, shorter) word, starting $L-1$ letters to the left of $u$ (or the beginning of $\mathrm{w}$ if the prefix before $u$ has length less than $L-1)$. After at most $\|\mathrm{w}\|$ steps, one will have one of the following cases:

- $\mathrm{w}$ is reduced to the empty word (in which case, w represents the identity of $H$ ), or

- a non-empty word defined by a L-local geodesic in the Cayley graph of $H$ (in this case, w does not represent the identity of $H$ because of Corollary 2.2.7).

Convention 4.1.8. In this subsection, the following data is considered input of the algorithms below:

- a group $G$ hyperbolic relative to the set of subgroups $\mathcal{P}=\left\{P_{1}, \cdots, P_{m}\right\}$, defined by a finite relative presentation

$$
\left\langle S_{0}, P_{1}, \cdots, P_{m} \mid R=1, R \in \mathcal{R}\right\rangle,
$$

and given finite generating sets $S_{1}, \cdots, S_{m}$ such that $P_{i}=\left\langle S_{i}\right\rangle$ for all $1 \leq i \leq m$ and $S=S_{0} \sqcup S_{1} \sqcup \cdots \sqcup S_{m}$, 
- a solution to the word problem in each $P_{i} \in \mathcal{P}$ of $G$.

In the Convention 4.0.1, we denote by $\widehat{S}=S_{0} \cup P_{1} \cup \cdots \cup P_{m}, \mathcal{A}=S^{ \pm 1}$ and $\mathcal{A}_{i}=S_{i}^{ \pm 1}$ for all $1 \leq i \leq m, \Gamma$ the Cayley graph of $G$ with respect to $S, \widehat{\Gamma}$ the coned-off Cayley graph of $G$ with respect to $\mathcal{P}$ (or $\widehat{S}$ ), and $\Gamma_{i}$ the Cayley graph of $P_{i}$ with respect to $S_{i}$ for all $1 \leq i \leq m$.

We discuss some of the preparation how we compute data which will be used in our algorithm, called a pre-computation.

Remark 4.1.9. The time complexity of the pre-computation will not be counted to the time complexity of our algorithm.

Theorem 4.1.10. With Convention 4.1.8, one can compute the following data:

1. the constants $\delta, C(\lambda, \epsilon, k)$ for any $\lambda \geq 1, \epsilon \geq 0$ and $k \geq 0, L=8 \delta+1$ and the constant $k(3,2 \delta)$ from Proposition 4.1.4;

2. the presentation

$$
\left\langle S_{0}, P_{1}, \cdots, P_{m} \mid R=1, R \in \mathcal{R}_{1}\right\rangle
$$

which is the relative Dehn presentation of $G$ with respect to $\mathcal{P}=\left\{P_{1}, \cdots, P_{m}\right\}$, where

$$
\mathcal{R}_{1}=\left\{R \in \mathcal{A}^{*} \mid R=1,\|R\|_{S} \leq(2 L-1) C(2)\right\}
$$

3. the set

$$
\mathcal{L}=\left\{(u, v) \in \mathcal{A}^{*} \times \mathcal{A}^{*} \mid u v^{-1} \in \mathcal{R}_{1},\|u\|_{\widehat{S}} \leq L,\|v\|_{\widehat{S}}<\|u\|_{\widehat{S}}\right\}
$$

Proof. In Dah08 Theorem 0.1, an explicit bound for the linear relative isoperimetric inequality satisfied by the given finite relative presentation 4.1 can be found. By using the explicit bound, we can apply the result from $\left[\mathrm{ABC}^{+}\right.$91] pages 33-35 or Theorem 2.9 in Chapter III.H of [BH99] to compute a hyperbolicity constant $\delta$ for $\widehat{\Gamma}$. See also Theorem 4.1 (2) in [Bum15]. Using Theorem 3.23 of [Osi06c, we are able to compute the constant $C(\lambda, \epsilon, k)$ (see also Theorem 4.1 (3) in [Bum15]). It follows that the constants $L=8 \delta+1$ and $k(3,2 \delta)$ can be computed.

The second assertion is discussed in Section 3.4 of [Far98]. We briefly describe it as follows. Let $\omega$ be a closed path in $\Gamma$. Without loss of generality, we can assume that every component of $\omega$ is geodesic in $\Gamma_{i}$ for some $1 \leq i \leq m$ because the word problem of each parabolic subgroup is solvable. We also can assume that $\widehat{\omega}$ is not a $L$-local geodesic in $\widehat{\Gamma}$; otherwise, $\omega_{-} \neq \omega_{+}$by Corollary 2.2.7, which contradicts to that $\omega$ is a closed path. So, there are a non-geodesic subpath $\widehat{\alpha}$ of $\widehat{\omega}$ such that $l_{\widehat{\Gamma}}(\widehat{\alpha}) \leq L$, and a geodesic $\widehat{\beta}$ in $\widehat{\Gamma}$ connecting the endpoints of $\widehat{\alpha}$. Moreover, $\widehat{\alpha}$ is $(2,0)$-quasigeodesic without backtracking and every component $\eta$ of $\alpha$ is isolated in the closed path $\alpha \beta^{-1}$ in $\Gamma$. By the BCP property for $(2,0)$-quasigeodesics, $l_{\Gamma}(\eta) \leq C(2)$. It follows that $l_{\Gamma}\left(\alpha \beta^{-1}\right) \leq(2 L-1) C(2)$. By taking $R=\phi(\alpha) \phi\left(\beta^{-1}\right)$, the result follows.

By using the finite set $\mathcal{R}_{1}$ from the second assertion, the third assertion is clear. All together, the statement is proven.

Definition 4.1.11. Suppose that each $P_{i} \in \mathcal{P}$ of $G$ has solvable word problem. Let $\omega$ be a path in $\Gamma$; let $\eta$ be a $P_{i}$-component of $\omega$ for some $P_{i} \in \mathcal{P}$. For a geodesic $\sigma$ in $\Gamma_{i}$ connecting the endpoints of $\eta$, the procedure of shortening the word $\phi(\eta) \in \mathcal{A}_{i}^{*}$ to the word $\phi(\sigma) \in \mathcal{A}_{i}^{*}$ is called a normalization. We say that $\phi(\eta)$ is normalized to $\phi(\sigma)$. 
In what follows, we introduce an algorithm solving the word problem for relatively hyperbolic groups in the spirit of Dehn's algorithm for hyperbolic groups described in Algorithm 4.1.7 and Farb's algorithm for relatively hyperbolic groups discussed in [Far98, Theorem 3.7]. In our algorithm, it may happen that a word $z_{1} u z_{2}$ is shortened to $z_{1} v z_{2}$, where $v, z_{1}$ and $z_{2}$ are $P_{i}$-syllables for some $P_{i} \in \mathcal{P}$ of $G$; and then we may apply a normalization procedure to $z_{1} v z_{2}$ depending on the length of a word $z$ with respect to $S$ which is a geodesic in $\Gamma_{i}$ and $z$ represents the same element of $G$ as $z_{1} v z_{2}$.

Theorem 4.1.12. With Convention 4.1.8, there is an algorithm that, given a word w in $\mathcal{A}^{*}$, decides whether $\mathrm{w}$ represents the identity of $G$.

Proof. Our algorithm consists of the following instructions.

(1) It converts w to a freely reduced word $\mathrm{w}_{0}$ and then normalizes every $P_{i}$-syllable of $\mathrm{w}_{0}$ for some $P_{i} \in \mathcal{P}$. Note that the normalization procedure can be done because each $P_{i} \in \mathcal{P}$ has the solvable word problem. Denote the edited word by $\mathrm{w}_{1}$ and the path in $\Gamma$ by $\omega_{1}$ such that $\phi\left(\omega_{1}\right)=\mathrm{w}_{1}$; and the algorithm goes to the next instruction.

(2) It searches from the left of $\mathrm{w}_{1}$ for a maximal subword $u$ from $\mathcal{L}$ in (4.3), i.e., $u$ is not a proper subword of another subword of $w_{1}$ which is also from $\mathcal{L}$; if the word $u$ is found, $u$ is replaced by the word $v$ such that $(u, v) \in \mathcal{L}$. Note that the set $\mathcal{L}$ was pre-computed by Theorem 4.1.10. Denote the edited word by $w_{2}$ and the path in $\Gamma$ by $\omega_{2}$ such that $\phi\left(\omega_{2}\right)=\mathrm{w}_{2}$. Let $\beta$ be the path in $\Gamma$ with $\phi(\beta)=v$. If there is no component of $\omega_{2}$ containing a subpath of $\beta$ in $\Gamma$, the algorithm proceeds the same as Algorithm 4.1.7, i.e., one goes back to the instruction (2) on the subword $\mathrm{w}^{\prime}=\phi\left(\omega^{\prime}\right)$ of $\mathrm{w}_{2}$, where $\omega_{-}^{\prime}$ is left to $\beta_{-}$and $l_{\Gamma}\left(\omega_{\omega_{-}^{\prime}, \beta_{-}}^{\prime}\right) \leq(2 L-1) C(2)$. If there exists a component $\sigma$ of $\omega_{2}$ containing a subpath of $\beta$ (possibly, $\beta$ is empty), then $\sigma$ is a concatenation of geodesics in $\Gamma_{j}$ for some $1 \leq j \leq m$, i.e., $\sigma=\sigma_{1} \sigma_{2} \cdots \sigma_{n}$ where each $\sigma_{i}$ is a geodesic in $\Gamma_{j}$; and the algorithm computes

$$
\left|l_{\Gamma}\left(\sigma_{k}\right)-\sum_{i \in I \backslash\{k\}} l_{\Gamma}\left(\sigma_{i}\right)\right|
$$

where $I=\{1,2, \cdots, n\}$ and $k \in I$ such that $l_{\Gamma}\left(\sigma_{k}\right)$ is maximal; and then it proceeds to the next instruction.

(3) If either

$$
\left|l_{\Gamma}\left(\sigma_{k}\right)-\sum_{i \in I \backslash\{k\}} l_{\Gamma}\left(\sigma_{i}\right)\right| \leq k(3,2 \delta)
$$

or $I=\{1, \cdots, n\}$ for $n \geq 3$ such that

$$
\sum_{i \in I \backslash\{k\}} l_{\Gamma}\left(\sigma_{i}\right)-l_{\Gamma}\left(\sigma_{k}\right)>k(3,2 \delta),
$$

then the algorithm applies the normalization procedure on $\phi(\sigma)$, and then goes to the instruction (2) searching for subwords of the edited word, starting $(2 L-1) C(2)$ letters to the left of $\phi(\sigma)$. Otherwise, the algorithm goes to the next instruction.

(4) If

$$
l_{\Gamma}\left(\sigma_{k}\right)-\sum_{i \in I \backslash\{k\}} l_{\Gamma}\left(\sigma_{i}\right)>k(3,2 \delta),
$$


then the algorithm does not normalize $\phi(\sigma)$. Note that

$$
d_{\Gamma}\left(\sigma_{-}, \sigma_{+}\right)>k(3,2 \delta) .
$$

In this case, the algorithm goes to the instruction (2) searching for subwords of the edited word from $(2 L-1) C(2)$ letters to the left of $\phi(\sigma)$ up to the last letter to the left of $\phi(\sigma)$, and then it goes to the instruction (2) searching for subwords starting the first letter to the right of $\phi(\sigma)$.

(5) When the algorithm reaches the end of the edited word, it displays the word, denoted by $w_{3}$. One applies the normalization procedure on $\mathrm{w}_{3}$, and then displays the word v. If $\mathrm{v}$ is empty, then the algorithm outputs that w represents the identity in $G$; otherwise, if $\mathrm{v}$ is non-empty, the algorithm outputs that $\mathrm{w}$ does not represent the identity in $G$.

Let $\nu$ be the path in $\Gamma$ such that $\phi(\nu)=\mathrm{v}$. Note that $\nu$ is a path connecting the end points of $\omega$. Because of the instruction (2), the inequality (4.7) from the instruction (4) and the final normalization procedure in the instruction (5), the path $\nu$ has one of the following possibilities:

(i) $\nu$ is empty,

(ii) $\widehat{\nu}$ is a non-empty $L$-local geodesic in $\widehat{\Gamma}$ such that every component of $\nu$ is a geodesic in some $\Gamma_{j}$, or

(iii) $\nu=\nu_{1} \circ \eta_{1} \circ \cdots \circ \nu_{n} \circ \eta_{n}$ is an alternate $\mathcal{C}(L)$-path in $\Gamma$, where each $\widehat{\nu}_{i} \in \mathcal{C}(L)$, each $\eta_{i}$ is a geodesic in some $\Gamma_{j}$ such that $d_{\Gamma}\left(\eta_{i_{-}}, \eta_{i_{+}}\right)>k(3,2 \delta)$.

If $\mathrm{v}$ is empty, i.e., the case (i) happens and it is clear that $\mathrm{w}$ represents the identity in $G$. If $\mathrm{v}$ is non-empty, then the case (ii) or (iii) can happen; and so, w does not represent the identity in $G$ because of Corollary 2.2.7 or the third assertion of Theorem 4.1.5, respectively.

\subsubsection{The time complexity of the algorithm}

Recall that the time complexity of an algorithm is the maximum amount of time taken by the algorithm to run on any input of size $n$. Throughout this subsection, we denote the algorithm in Theorem 4.1.12 by WP. We will discuss the time complexity of WP, provided the word problem in parabolic subgroups is solvable in time $f(n)$. We also assume that $f(n) \succeq \mathcal{O}(n)$ in this subsection.

Lemma 4.1.13. Let $\mathrm{w}$ be the input word of WP. Then the time complexity of the instruction (1) in WP is $f\left(\|\mathrm{w}\|_{S}\right)$.

Proof. Recall that the instruction (1) in WP is searching all parabolic syllables of $\mathrm{w}$ and applying the normalization procedure. Since $f(n) \succeq \mathcal{O}(n)$, its time complexity is

$$
\mathcal{O}\left(\|\mathrm{w}\|_{S}\right)+f\left(\|\mathrm{w}\|_{S}\right) \preceq f\left(2\|\mathrm{w}\|_{S}\right)=f\left(\|\mathrm{w}\|_{S}\right) .
$$

Lemma 4.1.14. Let $\mathrm{w}$ be the input word of WP. Then the number of replacements in the instruction (2) of WP is $\mathcal{O}\left(\|\mathrm{w}\|_{S}\right)$. 
Proof. Let $\mathrm{w}_{i}$ be the edited word after applied a replacement in the instruction (2) on $\mathrm{w}_{i-1}$ for all $i \geq 1$; and let each $\omega_{i}$ be the path in $\Gamma$ with $\phi\left(\omega_{i}\right)=\mathrm{w}_{i}$. Then $\mathrm{w}_{0}=\mathrm{w}$. It follows that $l_{\widehat{\Gamma}}\left(\widehat{\omega_{i-1}}\right) \nless l_{\widehat{\Gamma}}\left(\widehat{\omega}_{i}\right)$ for all $i \geq 1$. If $l_{\widehat{\Gamma}}\left(\widehat{\omega_{i-1}}\right)>l_{\widehat{\Gamma}}\left(\widehat{\omega}_{i}\right)$ for all $i \geq 1$, then the number of replacements in the instruction (2) is less than $\|\mathrm{w}\|_{S}$. Let us consider the other possibility that $l_{\widehat{\Gamma}}\left(\widehat{\omega_{i-1}}\right)=l_{\widehat{\Gamma}}\left(\widehat{\omega}_{i}\right)$ for all $i \geq 1$.

Let $u_{i-1}$ be a subword of $\mathrm{w}_{i-1}$ that is replaced by a subword $v_{i}$ of $\mathrm{w}_{i}$ for all $i \geq 1$ such that $\left(u_{i-1}, v_{i}\right) \in \mathcal{L}$ from (4.3); and let $\alpha_{i-1}$ and $\beta_{i}$ be the paths in $\Gamma$ such that $\phi\left(\alpha_{i-1}\right)=u_{i-1}$ and $\phi\left(\beta_{i}\right)=v_{i}$. Since $l_{\widehat{\Gamma}}\left(\widehat{\omega_{i-1}}\right)=l_{\widehat{\Gamma}}\left(\widehat{\omega}_{i}\right)$ and $l_{\widehat{\Gamma}}\left(\widehat{\alpha_{i-1}}\right)>l_{\widehat{\Gamma}}\left(\widehat{\beta}_{i}\right)$ for all $i \geq 1$, there exists a component $\eta$ of $\omega_{0}$ such that each $\alpha_{i-1}$ contains a subpath of $\eta$. Without loss of generality, we can assume that $\omega_{0}=\omega_{0}^{\prime} \circ \eta$, and $\alpha_{0}=\omega_{0}^{\prime} \circ \eta_{1}$ where $\eta_{1}$ is a subpath of $\eta$. Then for all $i \geq 1, \alpha_{i}$ is a subpath of $\omega_{i}$ containing a subpath of $\beta_{i}$. See Figure 4.3. It follows that $l_{\widehat{\Gamma}}\left(\widehat{\beta_{i+1}}\right)<l_{\widehat{\Gamma}}\left(\widehat{\beta}_{i}\right)$ for all $i \geq 1$. Since $l_{\widehat{\Gamma}}\left(\widehat{\beta}_{1}\right) \leq L-1$, we

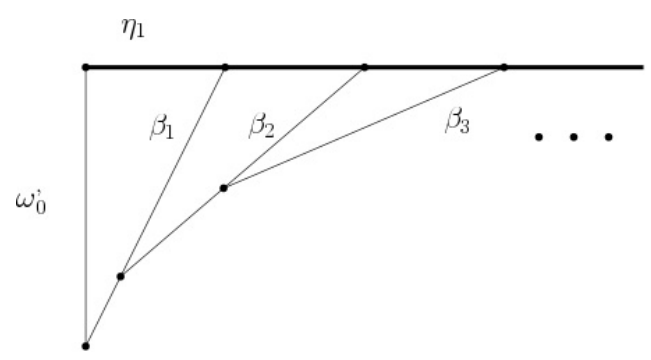

Figure 4.3: Instruction (2) in WP

have $i \leq L-1$. Therefore, the number of replacements in the instruction $(2)$ is $\mathcal{O}\left(l_{\widehat{\Gamma}}(\widehat{\omega})\right)$. Since $l_{\widehat{\Gamma}}(\widehat{\omega}) \leq\|\mathrm{w}\|_{S}$, the assertion follows.

Corollary 4.1.15. Let $\mathrm{w}$ and $\mathrm{v}$ be the input and output words of $\mathrm{WP}$, respectively. Then $\|\mathrm{v}\|_{S} \preceq \mathcal{O}\left(\|\mathrm{w}\|_{S}\right)$.

Proof. Recall that the length of each parabolic syllable of a replaced word with respect to $S$ is at most $C(2)$ in the definition of $\mathcal{L}$ from (4.3). By Lemma 4.1.14. $\|\mathrm{v}\|_{S} \preceq$ $\mathcal{O}\left(\|\mathrm{w}\|_{S}\right) C(2)=\mathcal{O}\left(\|\mathrm{w}\|_{S}\right)$.

In what follows, we will discuss the time complexity of WP for a certain input word.

Proposition 4.1.16. Suppose that

$$
\chi=\sigma_{1} \beta_{1} \sigma_{2} \beta_{2} \cdots \beta_{n-1} \sigma_{n}
$$

is a path in $\Gamma_{t}$ for some $1 \leq t \leq m$ such that the following properties hold:

- each $\sigma_{i}$ is a geodesic in $\Gamma_{t}$, and each $\beta_{i}$ (possibly, $\beta_{i}$ is empty) is a concatenation of geodesics $\zeta_{s}^{i}$ in $\Gamma_{t}$; and

- the word $\phi\left(\sigma_{1} \beta_{1} \sigma_{2}\right)$ is normalized by the instruction (3) of WP to $\phi\left(\eta_{2}\right)$, and the word $\phi\left(\eta_{j-1} \beta_{j-1} \sigma_{j}\right)$ is normalized by the instruction (3) of WP to $\phi\left(\eta_{j}\right)$ for all $3 \leq j \leq n$.

Then for each $2 \leq k \leq n$,

$$
l_{\Gamma}\left(\eta_{k}\right) \leq 2^{k-1} l_{\Gamma}\left(\sigma_{1}\right)+2^{k} \sum_{i=1}^{k-1} l_{\Gamma}\left(\beta_{i}\right)+2^{k-1} k(3,2 \delta) .
$$

Moreover, the time complexity $T_{\chi}$ of the normalization procedure in WP on the word $\phi(\chi)$ is $f\left(l_{\Gamma}(\chi)\right)$. 


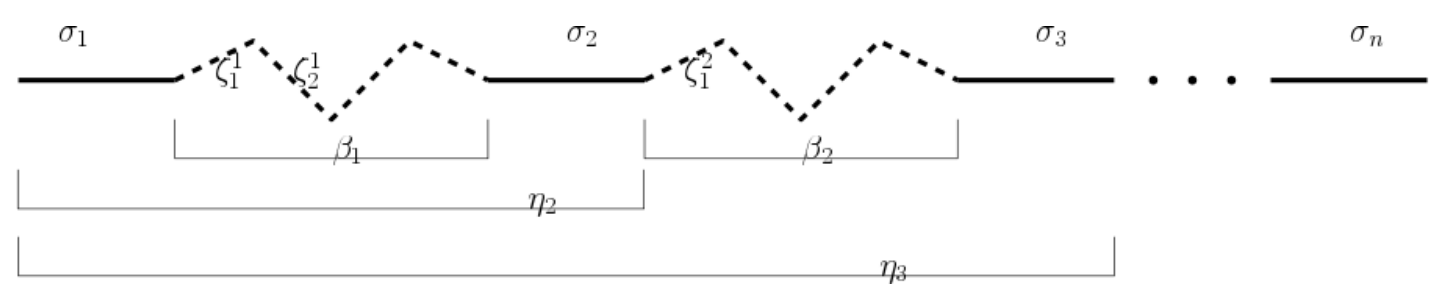

Figure 4.4: Normalizations on $\phi(\chi)$

Proof. See Figure 4.4. There are three cases to discuss: for all $2 \leq k \leq n$, the word $\phi\left(\eta_{k}\right)$ satisfies the inequality (4.4) only, the inequality (4.5) only, and a combination of the inequalities (4.4) and (4.5).

First, let us consider the case when the word $\phi\left(\eta_{k}\right)$ satisfies the inequality (4.4) for all $2 \leq k \leq n$. Then

$$
\begin{aligned}
l_{\Gamma}\left(\eta_{2}\right) & \leq l_{\Gamma}\left(\sigma_{1}\right)+l_{\Gamma}\left(\beta_{1}\right)+l_{\Gamma}\left(\sigma_{2}\right) \\
& \leq l_{\Gamma}\left(\sigma_{1}\right)+l_{\Gamma}\left(\beta_{1}\right)+\left[l_{\Gamma}\left(\sigma_{1}\right)+l_{\Gamma}\left(\beta_{1}\right)+k(3,2 \delta)\right] \text { by } 4.4 \\
& \leq 2 l_{\Gamma}\left(\sigma_{1}\right)+2 l_{\Gamma}\left(\beta_{1}\right)+k(3,2 \delta)
\end{aligned}
$$

and

$$
\begin{aligned}
l_{\Gamma}\left(\eta_{3}\right) & \leq l_{\Gamma}\left(\eta_{2}\right)+l_{\Gamma}\left(\beta_{2}\right)+l_{\Gamma}\left(\sigma_{3}\right) \\
& \left.\leq l_{\Gamma}\left(\eta_{2}\right)+l_{\Gamma}\left(\beta_{2}\right)+\left[l_{\Gamma}\left(\eta_{2}\right)+l_{\Gamma}\left(\beta_{2}\right)+k(3,2 \delta)\right] \text { by } 4.4\right) \\
& =2 l_{\Gamma}\left(\eta_{2}\right)+2 l_{\Gamma}\left(\beta_{2}\right)+k(3,2 \delta) \\
& \leq 2\left[2 l_{\Gamma}\left(\sigma_{1}\right)+2 l_{\Gamma}\left(\beta_{1}\right)+k(3,2 \delta)\right]+2 l_{\Gamma}\left(\beta_{2}\right)+k(3,2 \delta) \\
& =2^{2} l_{\Gamma}\left(\sigma_{1}\right)+2^{2} l_{\Gamma}\left(\beta_{1}\right)+2 l_{\Gamma}\left(\beta_{2}\right)+3 k(3,2 \delta) \\
& \leq 2^{2} l_{\Gamma}\left(\sigma_{1}\right)+\left(2+2^{2}\right)\left[l_{\Gamma}\left(\beta_{1}\right)+l_{\Gamma}\left(\beta_{2}\right)\right]+3 k(3,2 \delta) .
\end{aligned}
$$

By iterating, we have

$$
\begin{aligned}
l_{\Gamma}\left(\eta_{k}\right) & \leq 2^{k-1} l_{\Gamma}\left(\sigma_{1}\right)+\sum_{i=1}^{k-1} 2^{i} \sum_{i=1}^{k-1} l_{\Gamma}\left(\beta_{i}\right)+\left(2^{k-1}-1\right) k(3,2 \delta) \\
& \leq 2^{k-1} l_{\Gamma}\left(\sigma_{1}\right)+2^{k} \sum_{i=1}^{k-1} l_{\Gamma}\left(\beta_{i}\right)+2^{k-1} k(3,2 \delta) .
\end{aligned}
$$

Second, let us consider the case when the word $\phi\left(\eta_{k}\right)$ satisfies the inequality 4.5 only for all $2 \leq k \leq n$. Then

$$
\begin{aligned}
l_{\Gamma}\left(\eta_{2}\right) & \leq l_{\Gamma}\left(\sigma_{1}\right)+l_{\Gamma}\left(\beta_{1}\right)+l_{\Gamma}\left(\sigma_{2}\right) \\
& \leq l_{\Gamma}\left(\sigma_{1}\right)+l_{\Gamma}\left(\beta_{1}\right)+\left[l_{\Gamma}\left(\sigma_{1}\right)+l_{\Gamma}\left(\beta_{1}\right)\right] \text { by } \\
& \leq 2 l_{\Gamma}\left(\sigma_{1}\right)+2 l_{\Gamma}\left(\beta_{1}\right)
\end{aligned}
$$

and

$$
\begin{aligned}
l_{\Gamma}\left(\eta_{3}\right) & \leq l_{\Gamma}\left(\eta_{2}\right)+l_{\Gamma}\left(\beta_{2}\right)+l_{\Gamma}\left(\sigma_{3}\right) \\
& \left.\leq l_{\Gamma}\left(\eta_{2}\right)+l_{\Gamma}\left(\beta_{2}\right)+\left[l_{\Gamma}\left(\eta_{2}\right)+l_{\Gamma}\left(\beta_{2}\right)\right] \text { by } 4.5\right) \\
& \leq 2 l_{\Gamma}\left(\eta_{2}\right)+2 l_{\Gamma}\left(\beta_{2}\right) \\
& \leq 2\left[2 l_{\Gamma}\left(\sigma_{1}\right)+2 l_{\Gamma}\left(\beta_{1}\right)\right]+2 l_{\Gamma}\left(\beta_{2}\right) \\
& =2^{2} l_{\Gamma}\left(\sigma_{1}\right)+2^{2} l_{\Gamma}\left(\beta_{1}\right)+2 l_{\Gamma}\left(\beta_{2}\right) \\
& \leq 2^{2} l_{\Gamma}\left(\sigma_{1}\right)+\left(2+2^{2}\right)\left[l_{\Gamma}\left(\beta_{1}\right)+l_{\Gamma}\left(\beta_{2}\right)\right] .
\end{aligned}
$$


By iterating, we have

$$
\begin{aligned}
l_{\Gamma}\left(\eta_{k}\right) & \leq 2^{k-1} l_{\Gamma}\left(\sigma_{1}\right)+\sum_{i=1}^{k-1} 2^{i} \sum_{i=1}^{k-1} l_{\Gamma}\left(\beta_{i}\right) \\
& \leq 2^{k-1} l_{\Gamma}\left(\sigma_{1}\right)+2^{k} \sum_{i=1}^{k-1} l_{\Gamma}\left(\beta_{i}\right)+2^{k-1} k(3,2 \delta) .
\end{aligned}
$$

Next, we consider the last case when the word $\phi\left(\eta_{k}\right)$ satisfies the inequalities 4.4 and (4.5) for all $2 \leq k \leq n$. An induction on $r \in\{2, \cdots, n-1\}$ yields that

$$
\begin{aligned}
l_{\Gamma}\left(\eta_{r+1}\right) & \leq l_{\Gamma}\left(\eta_{r}\right)+l_{\Gamma}\left(\beta_{r}\right)+l_{\Gamma}\left(\sigma_{r+1}\right) \\
& \leq l_{\Gamma}\left(\eta_{r}\right)+l_{\Gamma}\left(\beta_{r}\right)+\left[l_{\Gamma}\left(\eta_{r}\right)+l_{\Gamma}\left(\beta_{r}\right)+k(3,2 \delta)\right] \text { by 4.4 and 4.5 } \\
& \leq 2 l_{\Gamma}\left(\eta_{r}\right)+2 l_{\Gamma}\left(\beta_{r}\right)+k(3,2 \delta) \\
& \leq 2\left[2^{r-1} l_{\Gamma}\left(\sigma_{1}\right)+\sum_{i=1}^{r-1} 2^{i} \sum_{i=1}^{r-1} l_{\Gamma}\left(\beta_{i}\right)+\left(2^{r-1}-1\right) k(3,2 \delta)\right]+2 l_{\Gamma}\left(\beta_{r}\right)+k(3,2 \delta) \\
& \leq 2^{r} l_{\Gamma}\left(\sigma_{1}\right)+2^{r+1} \sum_{i=1}^{r} l_{\Gamma}\left(\beta_{i}\right)+2^{r} k(3,2 \delta) .
\end{aligned}
$$

All together, we have the inequality (4.8). To compute the time complexity of the normalization procedure in WP on $\phi(\chi)$, we have

$$
\begin{aligned}
& \sum_{k=2}^{n} f\left(l_{\Gamma}\left(\eta_{k}\right)\right) \\
& \preceq f\left(\sum_{k=2}^{n} l_{\Gamma}\left(\eta_{k}\right)\right) \text { since } f(n) \succeq \mathcal{O}(n) \\
& \preceq f\left(\sum_{k=2}^{n}\left(2^{k-1} l_{\Gamma}\left(\sigma_{1}\right)+2^{k} \sum_{i=1}^{k-1} l_{\Gamma}\left(\beta_{i}\right)+2^{k-1} k(3,2 \delta)\right)\right) \text { by the inequality } 4.8 \\
& =f\left(l_{\Gamma}\left(\eta_{n}\right)\right) \text { by the equality } 4.8 \\
& \preceq f\left(l_{\Gamma}(\chi)\right) \text { because } l_{\Gamma}\left(\eta_{n}\right) \leq l_{\Gamma}(\chi) .
\end{aligned}
$$

Therefore, $T_{\chi}=f\left(l_{\Gamma}(\chi)\right)$.

Theorem 4.1.17. Suppose that $\mathrm{w}$ is the input word of WP. Then the time complexity of WP on $\mathrm{w}$ is $f\left(\|\mathrm{w}\|_{S}\right)$.

Proof. By Lemma 4.1.13, the time complexity of the instruction (1) in WP on w is $f\left(\|\mathrm{w}\|_{S}\right)$. Let $\omega$ be a path in $\Gamma$ labeled by w. Without loss of generality, we can assume that every component of $\omega$ is a geodesic in $\Gamma_{t}$ for some $1 \leq t \leq m$.

In the instruction (2) of WP, a subword $u$ of $\mathrm{w}$ is replaced by the word $v$ such that $(u, v) \in \mathcal{L}$ from (4.3). Let $\alpha$ and $\beta$ be the paths in $\Gamma$ such that $\phi(\alpha)=u$ and $\phi(\beta)=v$. Let $\mathrm{w}_{1}$ be the edited word after this replacement; and let $\omega_{1}$ be the path in $\Gamma$ such that $\phi\left(\omega_{1}\right)=\mathrm{w}_{1}$. Suppose that $\eta_{1}$ is a component of $\omega_{1}$ containing a subpath of $\beta$. Then $\eta_{1}$ has the following possibilities:

(1) the path $\beta$ is empty and $\eta_{1}=\sigma_{1} \sigma_{2}$, 
(2) the component $\eta_{1}=\sigma_{1} \sigma_{2}$ where either $\sigma_{1}$ or $\sigma_{2}$ is a component of $\beta$, or

(3) the component $\eta_{1}=\sigma_{1} \sigma_{2} \sigma_{3}$ where $\sigma_{2}=\beta$.

Let $\mathrm{w}_{i}$ be the edited word after one replacement for all $i \geq 1$; let $\omega_{i}$ be the path in $\Gamma$ such that $\mathrm{w}_{i}=\phi\left(\omega_{i}\right)$; and let $\eta_{i}$ be a component of the path $\omega_{i}$ corresponding to one of the above cases. See Figure 4.5 .

(1)

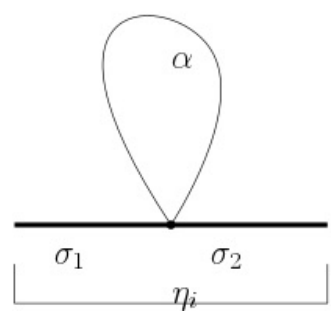

(2)

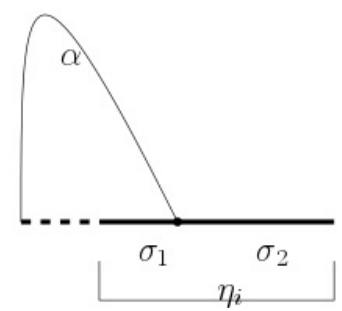

(3)

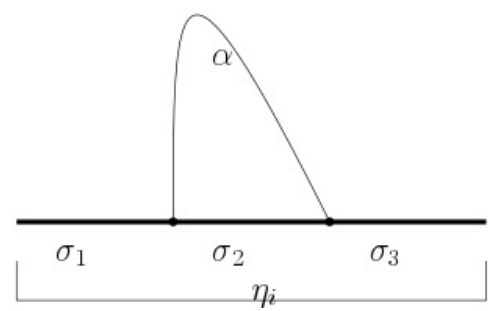

Figure 4.5: The syllable $\phi\left(\eta_{i}\right)$ of $\mathrm{w}_{i}$

By Proposition 4.1.16, the time complexity of the normalization procedure in WP on w is $f\left(\sum_{i} l_{\Gamma}\left(\eta_{i}\right)\right)$. Note that $\sum_{i} l_{\Gamma}\left(\eta_{i}\right) \preceq \mathcal{O}\left(\|\mathrm{w}\|_{S}\right)$ by Corollary 4.1.15. It follows that the time complexity of WP on $\mathrm{w}$ is $f\left(\|\mathrm{w}\|_{S}\right)$.

Let $\mathrm{w}$ be the input word of WP and let $n=\|\mathrm{w}\|_{S}$. We have one of main theorems in this thesis as follows.

Theorem 4.1.18. The time complexity $T(n)$ of the algorithm WP in Theorem 4.1.12 solving the word problem for relatively hyperbolic groups is

$$
T(n)=\max \{\mathcal{O}(n), f(n)\},
$$

provided that the word problem in each parabolic subgroup is solvable in time $f(n)$.

\subsection{The conjugacy problem}

\subsubsection{Estimates}

We will find some estimates for solving the conjugacy problem of $G$ in this subsection.

Definition 4.2.1. Let $\omega$ be a path in $\Gamma$; and let $\omega^{2}$ be the path in $\Gamma$ labeled by the word $\phi(\omega) \phi(\omega)$ in $\mathcal{A}^{*}$. Let $\omega=\omega_{1} \circ \omega_{2}$. We say that $\widehat{\omega^{\prime}}$ is a cyclic permutation of $\widehat{\omega}$ in $\widehat{\Gamma}$ if $\widehat{\omega^{\prime}}$ is a subpath of $\widehat{\omega^{2}}$ in $\widehat{\Gamma}$ such that the path $\omega^{\prime}$ in $\Gamma$ is labeled by the word $\phi\left(\omega_{2}\right) \phi\left(\omega_{1}\right)$ in $\mathcal{A}^{*}$. Let $L>1$ be an integer. We say that $\widehat{\omega}$ is a cyclic L-local geodesic in $\widehat{\Gamma}$ if $\widehat{\omega^{2}}$ is a $L$-local geodesic in $\widehat{\Gamma}$.

Remark 4.2.2. With the notation in the above definition, if $\omega_{c}$ is the path in $\Gamma$ such that $\phi\left(\omega_{c}\right)=\phi(\omega)$ and $\omega_{c-}=\omega_{+}$, then $\omega^{2}=\omega \omega_{c}$. In what follows, we denote by $\omega^{2}=\omega \omega_{c}$ in $\Gamma$. 
Definition 4.2.3. Let $\widehat{\alpha}$ and $\widehat{\beta}$ be paths in $\widehat{\Gamma}$. We say that $\widehat{\alpha}$ and $\widehat{\beta}$ form a conjugacy diagram $Q$ in $\widehat{\Gamma}$ if there exist non-empty geodesics $\widehat{\rho}$ and $\widehat{\rho^{\prime}}$ in $\widehat{\Gamma}$ such that $\phi(\rho)=\phi\left(\rho^{\prime}\right) \in$ $\mathcal{A}^{*}$ and $Q=\widehat{\rho} \widehat{\alpha} \widehat{\rho}^{\prime}-1 \widehat{\beta}^{-1}$ is a closed path in $\widehat{\Gamma}$ with 4 sides $\widehat{\rho}, \widehat{\alpha}, \widehat{\rho^{\prime}}$ and $\widehat{\beta}$; we denote by $Q_{\Gamma}$ the closed path $\rho \alpha \rho^{-1} \beta^{-1}$ in $\Gamma$. We say that the conjugacy diagram $Q$ is minimal if the following properties hold:

1. $\beta^{-1} \rho \alpha=\beta^{-1} \circ \rho \circ \alpha$; and

2. $l_{\widehat{\Gamma}}(\widehat{\gamma}) \geq l_{\widehat{\Gamma}}(\widehat{\rho})$ for any geodesic $\widehat{\gamma}$ in $\widehat{\Gamma}$ connecting a vertex of $\widehat{\alpha}$ and a vertex of $\widehat{\beta}$ so that the first condition holds.

Remark 4.2.4. If $Q=\widehat{\rho} \widehat{\alpha} \widehat{\rho}^{\prime}-1 \widehat{\beta}^{-1}$ is a minimal conjugacy diagram in $\widehat{\Gamma}$ such that $\phi(\rho) \in \mathcal{A}_{i}^{*}$ for some $1 \leq i \leq m$, then $\rho$ is a component of $Q_{\Gamma}$.

Lemma 4.2.5. Let $\widehat{\alpha}$ and $\widehat{\beta}$ be paths in $\widehat{\Gamma}$. If $\widehat{\alpha}$ and $\widehat{\beta}$ form a conjugacy diagram in $\widehat{\Gamma}$, then there exists a minimal conjugacy diagram $Q=\widehat{\rho} \widehat{\alpha}^{\prime} \widehat{\rho}^{\prime}-1 \widehat{\beta}^{-1}$ in $\widehat{\Gamma}$, where $\widehat{\alpha}^{\prime}$ and $\widehat{\beta}^{\prime}$ are cyclic permutations of $\widehat{\alpha}$ and $\widehat{\beta}$, respectively. If $\alpha^{\prime}$ and $\beta^{\prime}$ are paths without backtracking in $\Gamma$, then $\rho \circ \alpha^{\prime}, \rho^{-1} \circ \beta^{\prime-1}, \alpha^{\prime} \rho^{-1}$ and $\beta^{\prime} \rho^{\prime}$ are paths without backtracking in $\Gamma$.

Proof. The first statement is clear. If $\rho$ is a component of $Q_{\Gamma}$, then the second assertion follows from the assumption that $\alpha^{\prime}$ and $\beta^{\prime}$ are paths without backtracking in $\Gamma$. Otherwise, the second assertion follows from the minimality of $Q$.

Let us recall some results in hyperbolic groups which are modified from Corollary 3.2 of [Bum15], and Lemma 3.6 of [Bum15] or Lemma 2.11 Chapter III.Г of [BH99].

Lemma 4.2.6. [Bum15, Corollary 3.2] Let $L>8 \delta$; and let $\widehat{\alpha}$ and $\widehat{\beta}$ be two L-local geodesics in $\widehat{\Gamma}$. Suppose that $\widehat{\alpha}$ and $\widehat{\beta}$ form a conjugacy diagram $Q$ in $\widehat{\Gamma}$. Then each side of $Q$ is contained in $7 \delta$-neighbourhood of the other three sides.

Lemma 4.2.7. Bum15, Lemma 3.6] Let $L=8 \delta+1$; and let $\widehat{\alpha}$ and $\widehat{\beta}$ be cyclic L-local geodesics in $\widehat{\Gamma}$. Suppose that $\widehat{\alpha}$ and $\widehat{\beta}$ form a minimal conjugacy diagram $Q=\widehat{\rho} \widehat{\alpha} \widehat{\rho}^{\prime}-1 \widehat{\beta}^{-1}$ in $\widehat{\Gamma}$. Then

1. $\max \left\{l_{\widehat{\Gamma}}(\widehat{\alpha}), l_{\widehat{\Gamma}}(\widehat{\beta})\right\} \leq 86 \delta+3$, or else

2. there exist cyclic permutations $\widehat{\alpha^{\prime}}$ and $\widehat{\beta^{\prime}}$ of $\widehat{\alpha}$ and $\widehat{\beta}$, respectively, such that $\widehat{\alpha^{\prime}}$ and $\widehat{\beta}^{\prime}$ form a conjugacy diagram $Q^{\prime}=\widehat{\sigma}{\widehat{\alpha^{\prime}}}^{\widehat{\sigma}^{\prime}}{ }^{-1}{\widehat{\beta^{\prime}}}^{-1}$ in $\widehat{\Gamma}$ with $l_{\widehat{\Gamma}}(\widehat{\sigma}) \leq 7 \delta+1$.

The following lemma modified from Lemma 7.3 of [BH05] shows that $\widehat{\beta}^{\prime}$ in the second assertion of Lemma 4.2.7 is not needed. We also provide a proof for completeness.

Lemma 4.2.8. BH05, Lemma 7.3] Let $L=8 \delta+1$; and let $\widehat{\alpha}$ and $\widehat{\beta}$ be cyclic L-local geodesics in $\widehat{\Gamma}$. Suppose that $\widehat{\alpha}$ and $\widehat{\beta}$ form a minimal conjugacy diagram $Q=\widehat{\rho} \widehat{\alpha \rho^{\prime}} \widehat{\beta}^{-1}$ in $\widehat{\Gamma}$. Then

1. $\max \left\{l_{\widehat{\Gamma}}(\widehat{\alpha}), l_{\widehat{\Gamma}}(\widehat{\beta})\right\} \leq 86 \delta+3$, or else

2. there exists a cyclic permutation $\widehat{\alpha^{\prime \prime}}$ of $\widehat{\alpha}$ such that $\widehat{\alpha^{\prime \prime}}$ and $\widehat{\beta}$ form a conjugacy diagram $Q^{\prime \prime}=\widehat{\tau} \widehat{\alpha^{\prime \prime}} \widehat{\tau}^{\prime}-1 \widehat{\beta}^{-1}$ in $\widehat{\Gamma}$ with $l_{\widehat{\Gamma}}(\widehat{\tau}) \leq 21 \delta+3$. 
Proof. Suppose that $\max \left\{l_{\widehat{\Gamma}}(\widehat{\alpha}), l_{\widehat{\Gamma}}(\widehat{\beta})\right\}>86 \delta+3$. Then there exists a minimal conjugacy diagram $Q^{2}=\widehat{\rho} \alpha^{2}{\widehat{\rho^{\prime \prime}}}^{-1}{\widehat{\beta^{2}}}^{-1}$ in $\widehat{\Gamma}$ because $Q$ in Lemma 4.2.7 is a minimal conjugacy diagram. Note that $\beta^{\prime}$ in $Q_{\Gamma}^{\prime}=\sigma \alpha^{\prime} \sigma^{\prime-1} \beta^{\prime-1}$ of Lemma 4.2.7 is a subpath of $\beta^{2}$ in $\Gamma$ containing $\beta_{+}$. As $\beta_{+}$may be a vertex in $\Gamma_{t}$ for some $1 \leq t \leq m$, Lemma 4.2.6 implies that $d_{\widehat{\Gamma}}\left(\widehat{\beta}_{+}, x\right) \leq 7 \delta+1$ for some vertex $x \in \widehat{\sigma} \cup \widehat{\alpha^{\prime}} \cup \widehat{\sigma^{\prime}}$. Note that $d_{\widehat{\Gamma}}\left(\widehat{\sigma}_{-}, \widehat{\sigma}_{+}\right) \leq 7 \delta+1$ in $Q^{\prime}$ by Lemma 4.2.7. See Figure 4.6.

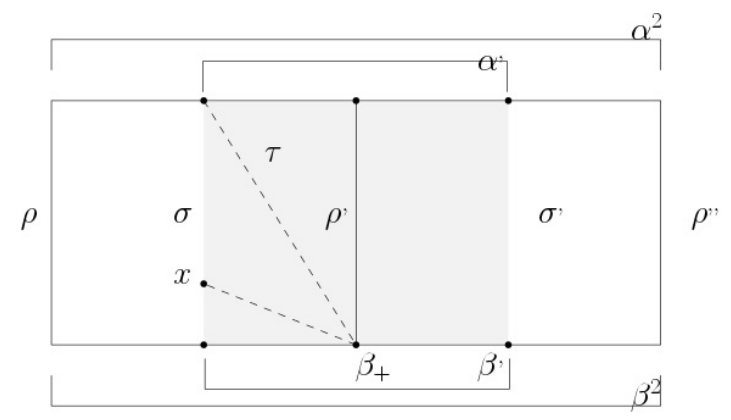

Figure 4.6: A minimal conjugacy diagram $Q^{2}$ in $\widehat{\Gamma}$

If $x \in \widehat{\sigma}$, then $d_{\widehat{\Gamma}}\left(\widehat{\beta}_{+}, \widehat{\beta}^{\prime}{ }_{-}\right) \leq d_{\widehat{\Gamma}}\left(\widehat{\beta}_{+}, x\right)+d_{\widehat{\Gamma}}\left(\widehat{\beta}^{\prime}, x\right) \leq d_{\widehat{\Gamma}}\left(\widehat{\beta}_{+}, x\right)+d_{\widehat{\Gamma}}\left(\widehat{\sigma}_{-}, \widehat{\sigma}_{+}\right) \leq 14 \delta+2$; and so, $d_{\widehat{\Gamma}}\left(\widehat{\beta}_{+}, \widehat{\sigma}_{+}\right) \leq d_{\widehat{\Gamma}}\left(\widehat{\beta}_{+}, \widehat{\beta}^{\prime}{ }_{-}\right)+d_{\widehat{\Gamma}}\left(\widehat{\sigma}_{-}, \widehat{\sigma}_{+}\right) \leq 21 \delta+3$. For $x \in \widehat{\sigma^{\prime}}$, it is analogous to show $d_{\widehat{\Gamma}}\left(\widehat{\beta}_{+},{\widehat{\sigma^{\prime}}}_{+}\right) \leq 21 \delta+3$. Thus, by taking $\widehat{\tau}$ or $\widehat{\tau^{\prime}}$ to be $\left[\widehat{\beta}_{+}, y\right]$ for $y \in\left\{\widehat{\sigma}_{+},{\widehat{\sigma^{\prime}}}_{+}, x\right\}$ where the vertex $x \in \widehat{\alpha^{\prime}}$ such that $d_{\widehat{\Gamma}}\left(\widehat{\beta}_{+}, x\right) \leq 7 \delta+1$, the second assertion follows.

Lemma 4.2.9. With the notation and assumptions of Lemma 4.2.ף. if $\max \left\{l_{\widehat{\Gamma}}(\widehat{\alpha}), l_{\widehat{\Gamma}}(\widehat{\beta})\right\}>$ $86 \delta+3$, then no component of $\rho$ is connected to a component of $\rho^{\prime}$. Moreover, if $\rho$ is a component of $Q_{\Gamma}$, then $d_{\Gamma}\left(\rho_{-}, \rho_{+}\right) \leq C(3,2 \delta, 1)$.

Proof. Suppose that there is a component of $\rho$ connected to a component of $\rho^{\prime}$. Note that $l_{\widehat{\Gamma}}(\widehat{\rho})=l_{\widehat{\Gamma}}\left(\widehat{\rho^{\prime}}\right) \leq 7 \delta+1$ by Lemma 4.2.7. Then $d_{\widehat{\Gamma}}\left(\widehat{\alpha}_{-}, \widehat{\alpha}_{+}\right) \leq l_{\widehat{\Gamma}}(\widehat{\rho})+l_{\widehat{\Gamma}}\left(\widehat{\rho}^{\prime}\right)+1=14 \delta+3$, and similarly $d_{\widehat{\Gamma}}\left(\widehat{\beta}_{-}, \widehat{\beta}_{+}\right) \leq 14 \delta+3$. Note that $\widehat{\alpha}$ and $\widehat{\beta}$ are $(3,2 \delta)$-quasigeodesics in $\widehat{\Gamma}$ by Corollary 2.2.6. So, $\max \left\{l_{\widehat{\Gamma}}(\widehat{\alpha}), l_{\widehat{\Gamma}}(\widehat{\beta})\right\} \leq 44 \delta+9$, which contradicts to our assumption $\max \left\{l_{\widehat{\Gamma}}(\widehat{\alpha}), l_{\widehat{\Gamma}}(\widehat{\beta})\right\}>86 \delta+3$. Hence, the first statement holds.

If $\rho$ is a component of $Q_{\Gamma}$, then $\rho$ is not connected to $\rho^{\prime}$ in $Q_{\Gamma}$ by the first assertion. Note that $\alpha$ and $\beta$ are paths without backtracking in $\Gamma$ by Lemma 3.1.6. By Lemma 4.2.5, $\rho$ is a component isolated in $Q_{\Gamma}$. It follows from Lemma 3.1 .7 that $d_{\Gamma}\left(\rho_{-}, \rho_{+}\right) \leq$ $C(3,2 \delta, 1)$. So the statement is proven.

Proposition 4.2.10. Let $L=8 \delta+1$; and let $\widehat{\alpha}$ and $\widehat{\beta}$ be cyclic L-local geodesics in $\widehat{\Gamma}$. Then there exists a constant $D=D(\delta)$ such that the following condition holds. If $\widehat{\alpha}$ and $\widehat{\beta}$ form a minimal conjugacy diagram $Q=\widehat{\rho} \widehat{\alpha}{\widehat{\rho^{\prime}}}^{-1} \widehat{\beta}^{-1}$ in $\widehat{\Gamma}$ and $\max \left\{l_{\widehat{\Gamma}}(\widehat{\alpha}), l_{\widehat{\Gamma}}(\widehat{\beta})\right\}>86 \delta+3$, then there exists a cyclic permutation $\widehat{\alpha^{\prime \prime}}$ of $\widehat{\alpha}$ such that $\widehat{\alpha^{\prime \prime}}$ and $\widehat{\beta}$ form a conjugacy diagram $Q^{\prime \prime}=\widehat{\tau} \widehat{\alpha^{\prime \prime}} \widehat{\tau}^{\prime}-1 \widehat{\beta}^{-1}$ in $\widehat{\Gamma}$ with $l_{\Gamma}(\tau) \leq D$.

Proof. Suppose that there exists a component of $\alpha$ connected to a component of $\beta$ in $Q_{\Gamma}$. The minimality of $Q$ implies that $l_{\widehat{\Gamma}}(\widehat{\rho})=1$. Then either $\phi(\rho) \in S_{0}$ or $\rho$ is a component of $Q_{\Gamma}$. If $\phi(\rho) \in S_{0}$, then $l_{\Gamma}(\rho)=1$. When $\rho$ is a component of $Q_{\Gamma}, d_{\Gamma}\left(\rho_{-}, \rho_{+}\right) \leq C(3,2 \delta, 1)$ from Lemma 4.2.9.

Next, suppose that no component of $\alpha$ is connected to a component of $\beta$ in $Q_{\Gamma}$. The existence of $Q^{\prime \prime}$ with $l_{\widehat{\Gamma}}(\widehat{\tau}) \leq 21 \delta+3$ is proven in Lemma 4.2.8. Let $\eta$ be a component of 
$\tau$. Note that $\alpha_{+} \in \alpha^{\prime \prime}$. Let $T=\tau \alpha_{\alpha_{-}^{\prime \prime}, \alpha_{+}}^{\prime \prime} \rho^{-1}$ be the closed path in $\Gamma$. Then $\alpha_{\alpha_{-}^{\prime \prime}, \alpha_{+}}^{\prime \prime} \rho^{-1}$ in $T$ is a path without backtracking; otherwise, the path $\alpha \rho^{-1}$ in $Q_{\Gamma}$ has a backtracking, which contradicts to Lemma 4.2.5. If $\eta$ is isolated in $T$, then $d_{\Gamma}\left(\eta_{-}, \eta_{+}\right) \leq C(1,0,21 \delta+3)$ by Lemma 3.1.7. If $\eta$ is connected to a component $\zeta$ of $T$, then $\zeta$ is a component of $\alpha_{\alpha_{-}^{\prime \prime}, \alpha_{+}}^{\prime \prime} \rho^{-1}$. Lemma 3.1.7 implies that $\max \left\{d_{\Gamma}\left(\eta_{+}, \zeta_{-}\right), d_{\Gamma}\left(\eta_{-}, \zeta_{+}\right)\right\} \leq C(1,0,21 \delta+3)$. Note that there exists a component $\zeta^{\prime}$ of $\alpha_{c} \rho^{\prime-1}$ with $\phi\left(\zeta^{\prime}\right)=\phi(\zeta)$. Because of the minimality of $Q$, Lemma 4.2.5. Lemma 4.2.9 and the assumption that no component of $\alpha$ is connected to a component of $\beta$ in $Q_{\Gamma}$, either $\zeta$ or $\zeta^{\prime}$ is isolated in $Q_{\Gamma}$. See Figure 4.7. So,

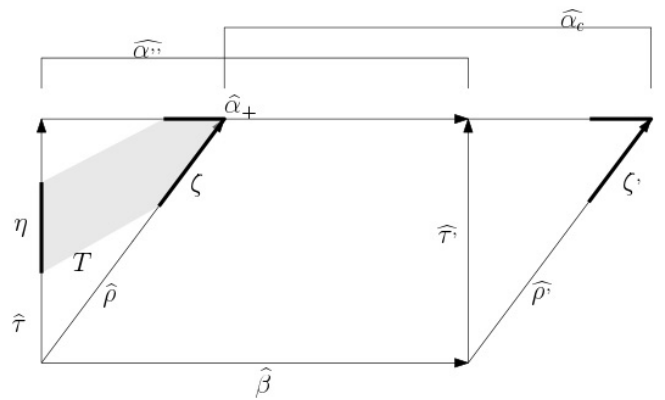

Figure 4.7: A conjugacy diagram $Q^{\prime \prime}$ in $\widehat{\Gamma}$

$d_{\Gamma}\left(\zeta_{-}, \zeta_{+}\right) \leq C(1,0,21 \delta+3)$ by Lemma 3.1.7. Therefore, $d_{\Gamma}\left(\eta_{-}, \eta_{+}\right) \leq 3 C(1,0,21 \delta+3)$; and hence, $l_{\Gamma}(\tau) \leq(21 \delta+3) 3 C(1,0,21 \delta+3)$.

Therefore, $l_{\Gamma}(\tau) \leq \max \{C(3,2 \delta, 1),(63 \delta+9) C(1,0,21 \delta+3)\}$; and so, the statement is proven.

\subsubsection{The algorithm and complexity}

In this subsection, we establish an algorithm providing the solution to the conjugacy problem for relatively hyperbolic groups, whenever solutions of the word and conjugacy problems of each parabolic subgroup are given; we also discuss the time complexity of the algorithm.

Convention 4.2.11. The following data is considered the inputs for the algorithms described in this subsection:

- a group $G$ hyperbolic relative to the set of subgroups $\mathcal{P}=\left\{P_{1}, \cdots, P_{m}\right\}$, presented by a relative Dehn presentation

$$
\left\langle S_{0}, P_{1}, \cdots, P_{m} \mid R=1, R \in \mathcal{R}\right\rangle,
$$

and given finite generating sets $S_{1}, \cdots, S_{m}$ such that $P_{i}=\left\langle S_{i}\right\rangle$ for all $1 \leq i \leq m$,

- a solution to the word problem in each $P_{i} \in \mathcal{P}$ of $G$; and let $f(n)$ be the time complexity of the word problem of parabolic subgroups $\mathcal{P}$ of $G$.

The following results are due to Bumagin Bum15. Our solution to the conjugacy problem in relatively hyperbolic groups is based on Bumagin's results, and our algorithm WP in Theorem 4.1.12 and Proposition 4.2.10. 
Lemma 4.2.12. Bum15, Proposition 5.3] With Convention 4.2.11, there is an algorithm such that, given a word $\mathrm{w}$ in $\mathcal{A}^{*}$, outputs words $\mathrm{w}^{\prime}$ and $\mathbf{z}$ in $\mathcal{A}^{*}$ such that $\mathrm{w}^{\prime}$ is the label of a cyclic L-local geodesic in $\widehat{\Gamma}$ with $\mathrm{w}^{\prime}=\mathrm{z}^{-1} \mathrm{wz}$ and $\|\mathrm{z}\|_{S} \leq C(2)\|\mathrm{w}\|_{S}$. The time complexity of the algorithm is $\|\mathrm{w}\|_{S} f\left(\|\mathrm{w}\|_{S}\right)$.

Lemma 4.2.13. [Bum15, Theorem 5.4] With Convention 4.2.11, there is an algorithm such that, given a word $\mathrm{w}$ in $\mathcal{A}^{*}$ representing an element $x$ in $G$, decides whether or not $x$ is a hyperbolic or parabolic element of $G$. The time complexity of the algorithm is $\|\mathrm{w}\|_{S} f\left(\|\mathrm{w}\|_{S}\right)$.

Lemma 4.2.14. Bum15, Lemma 5.7] Let $\widehat{\alpha}$ and $\widehat{\beta}$ be cyclic L-local geodesics in $\widehat{\Gamma}$; and let $\mathrm{u}=\phi(\widehat{\alpha})$ and $\mathrm{v}=\phi(\widehat{\beta})$. Suppose that $\max \left\{l_{\widehat{\Gamma}}(\widehat{\alpha}), l_{\widehat{\Gamma}}(\widehat{\beta})\right\}<86 \delta+3$, and that $\mathrm{u}$ and $\mathrm{v}$ represent hyperbolic elements of $G$. If $\mathrm{u}$ and $\mathrm{v}$ represent conjugate elements in $G$, then the following holds. There exist cyclic permutations $\widehat{\alpha^{\prime}}$ and $\widehat{\beta}^{\prime}$ of $\widehat{\alpha}$ and $\widehat{\beta}$, respectively, such that $\widehat{\alpha^{\prime}}$ and $\widehat{\beta^{\prime}}$ form a conjugacy diagram $Q^{\prime}=\widehat{\sigma}{\widehat{\alpha^{\prime}}}_{\sigma^{\prime}}^{-1}{\widehat{\beta^{\prime}}}^{-1}$ in $\widehat{\Gamma}$ with $l_{\Gamma}(\sigma) \leq K$ for some constant $K$.

Lemma 4.2.15. [Bum15, Theorem 5.8 (3)] With Convention 4.2.11, and suppose that a solution to the conjugacy problem in each $P_{i} \in \mathcal{P}$ of $G$ is given; and let $g(n)$ be the time complexity of the conjugacy problem of parabolic subgroups $\mathcal{P}$ of $G$. Then there is an algorithm such that, given words $\mathrm{u}$ and $\mathrm{v}$ in $\mathcal{A}^{*}$ representing parabolic elements of $G$, decides whether or not $\mathrm{u}$ and $\mathrm{v}$ represent conjugate elements in $G$. The time complexity of the algorithm is $\max \{n f(n), g(n)\}$, where $n=\|\mathrm{u}\|_{S}+\|\mathrm{v}\|_{S}$.

We are able to obtain one of the main results of this thesis as follows.

Theorem 4.2.16. With Convention 4.2.11, and suppose that a solution to the conjugacy problem in each $P_{i} \in \mathcal{P}$ of $G$ is given; and let $g(n)$ be the time complexity of the conjugacy problem of parabolic subgroups $\mathcal{P}$ of $G$. Then there is an algorithm such that, given words $\mathrm{u}$ and $\mathrm{v}$ in $\mathcal{A}^{*}$, decides whether or not $\mathrm{u}$ and $\mathrm{v}$ represent conjugate elements in $G$. Moreover, if $\mathrm{u}$ and $\mathrm{v}$ represent conjugate elements in $G$ which are hyperbolic, then the algorithm outputs a word in $\mathcal{A}^{*}$ that represents a conjugating element. The time complexity of the algorithm is $\max \left\{\mathcal{O}\left(n^{2}\right), n f(n), g(n)\right\}$, where $n=\|\mathrm{u}\|_{S}+\|\mathrm{v}\|_{S}$.

Proof. The algorithm consists of the following instructions.

(1) Using the algorithm in Lemma 4.2.12, one can convert $\mathrm{u}$ and $\mathrm{v}$ to words in $\mathcal{A}^{*}$ which are labels of cyclic $L$-local geodesics in $\widehat{\Gamma}$, denoted by $\widehat{\alpha}$ and $\widehat{\beta}$, respectively; and then it goes to the next instruction. By Lemma 4.2.12, the time complexity of this instruction is $n f(n)$.

(2) One computes $\max \left\{l_{\widehat{\Gamma}}(\widehat{\alpha}), l_{\widehat{\Gamma}}(\widehat{\beta})\right\}$ and uses the algorithm in Lemma 4.2 .13 to determine whether the words $\phi(\alpha)$ and $\phi(\beta)$ represent hyperbolic or parabolic elements in $G$. The time complexity of this instruction is $n f(n)$. If one of the elements is hyperbolic and the other is a parabolic element of $G$, the algorithm terminates and concludes that $\mathrm{u}$ and $\mathrm{v}$ do not represent conjugate elements in $G$. Otherwise, it goes to the next instruction.

(3) If $\max \left\{l_{\widehat{\Gamma}}(\widehat{\alpha}), l_{\widehat{\Gamma}}(\widehat{\beta})\right\}>86 \delta+3$, then for each cyclic permutation $\widehat{\alpha^{\prime}}$ of $\widehat{\alpha}$ in $\widehat{\Gamma}$ and every word q with $\|\mathrm{q}\|_{S} \leq D$ from Proposition 4.2 .10 represented by $\tau$ a path in $\Gamma$ with $\phi(\tau)=\mathrm{q}$, one applies the algorithm WP in Theorem 4.1 .12 to check whether 
the word $\mathrm{x}=\phi\left(\tau \alpha^{\prime} \tau^{-1} \beta^{-1}\right)$ in $\mathcal{A}^{*}$ represents the identity of $G$. If $\mathrm{x}$ represents the identity in $G$, then the procedure concludes that $\mathrm{u}$ and $\mathrm{v}$ represent conjugate elements in $G$; and then it outputs the word $\mathrm{w}=\mathrm{qy}^{-1} \mathrm{z}^{-1}$ and terminates, where $\mathrm{y}=\phi\left(\alpha_{\alpha_{-}, \alpha_{-}^{\prime}}\right)$ and $\mathrm{z}$ is given in Lemma 4.2.12. Theorem 4.1.18 implies that the time complexity of this case is $\max \left\{\mathcal{O}\left(n^{2}\right), n f(n)\right\}$.

Suppose that $\max \left\{l_{\widehat{\Gamma}}(\widehat{\alpha}), l_{\widehat{\Gamma}}(\widehat{\beta})\right\} \leq 86 \delta+3$ and that both $\phi(\alpha)$ and $\phi(\beta)$ represent hyperbolic elements in $G$. Then for each word $\phi(\sigma) \in \mathcal{A}^{*}$ with $\|\phi(\sigma)\|_{S} \leq K$ and each cyclic permutation $\widehat{\alpha^{\prime}}$ and $\widehat{\beta}^{\prime}$ of $\widehat{\alpha}$ and $\widehat{\beta}$, respectively, in $\widehat{\Gamma}$ provided in Lemma 4.2.14, one applies the algorithm WP to determine whether or not the word $\mathrm{p}=\phi\left(\sigma \alpha^{\prime} \sigma^{\prime-1} \beta^{\prime-1}\right)$ represents the identity of $G$. Note that the number of cyclic permutations $\widehat{\alpha^{\prime}}$ and $\widehat{\beta^{\prime}}$ are bounded by a constant. The time complexity of this case is $\max \{\mathcal{O}(n), f(n)\}$ by Theorem 4.1.18. If none of those words represent the identity of $G$, the algorithm terminates and concludes that $\mathrm{u}$ and $\mathrm{v}$ do not represent conjugate elements in $G$. If the word $\mathrm{p}=\phi\left(\sigma \alpha^{\prime} \sigma^{\prime-1} \beta^{\prime-1}\right)$ represents the identity in $G$, then the procedure concludes that $\mathrm{u}$ and v represent conjugate elements in $G$; and then it outputs the word $\mathrm{w}=\mathrm{py}^{-1} \mathrm{z}^{-1}$, and terminates, where $\mathrm{y}=\phi\left(\alpha_{\alpha_{-}, \alpha_{-}^{\prime}}\right)$ and $\mathrm{z}$ is given in Lemma 4.2 .12 .

If both $\phi(\alpha)$ and $\phi(\beta)$ represent parabolic elements in $G$, then one applies the algorithm in Lemma 4.2.15 and the time complexity is $\max \{n f(n), g(n)\}$.

All together, the time complexity of the algorithm is $\max \left\{\mathcal{O}\left(n^{2}\right), n f(n), g(n)\right\}$. 


\section{Chapter 5}

\section{Residual property of relatively hyperbolic groups}

This chapter is based on the work of [BZ16]. In this chapter, we will use the definition of a relatively hyperbolic group introduced by Osin (see Subsection 2.3.1). We say that $G$ is a toral relatively hyperbolic group if $G$ is torsion-free hyperbolic relative to a collection of abelian subgroups. We denote by $\mathscr{R}$ the class of finitely generated toral relatively hyperbolic groups.

\subsection{Residual homomorphisms}

Let us introduce the following two definitions of residual properties for a group. In this section, we will prove that Definitions 5.1.1 and 5.1.2 over the class $\mathscr{R}$ are equivalent. Our proof is built upon results of Ol'shanskii and Osin. In Osi10, Osin generalizes the small cancellation theory over hyperbolic groups, developed by Ol'shanskii in [Ol'93, to relatively hyperbolic groups. See the discussion in Section 3.3 .

Definition 5.1.1 (Classical Definition). Let $\mathcal{C}$ be a class of groups. A group $G$ is called a residually-C $\mathcal{C}$ group if for every nontrivial element $1 \neq g \in G$ there is a group $H_{g} \in \mathcal{C}$ and an onto homomorphism $\phi_{g}: G \rightarrow H_{g}$, such that $\phi_{g}(g) \neq 1$. A group $G$ is called a fully residually-C group if for every finite set $S=\left\{g_{1}, \ldots, g_{n}\right\}$ of distinct elements of $G$ there is a group $H_{S} \in \mathcal{C}$ and an onto homomorphism $\phi_{S}: G \rightarrow H_{S}$, such that the images $\phi_{S}\left(g_{1}\right), \ldots, \phi_{S}\left(g_{n}\right)$ are all distinct in $H_{S}$.

Definition 5.1.2 (Working Definition). Let $\mathcal{C}$ be a class of groups. A group $G$ is called a residually-C group if for every nontrivial element $1 \neq g \in G$ there is a group $H_{g} \in \mathcal{C}$ and a homomorphism $\phi_{g}: G \rightarrow H_{g}$, such that $\phi_{g}(g) \neq 1$. A group $G$ is called a fully residually-C group if for every finite set $S=\left\{g_{1}, \ldots, g_{n}\right\}$ of distinct elements of $G$ there is a group $H_{S} \in \mathcal{C}$ and a homomorphism $\phi_{S}: G \rightarrow H_{S}$, such that the images $\phi_{S}\left(g_{1}\right), \ldots, \phi_{S}\left(g_{n}\right)$ are all distinct in $H_{S}$.

The following theorem is a part of Theorem 3.3 .4 .

Theorem 5.1.3 (Osin). Let $G$ be a group hyperbolic relative to a collection of subgroups $\left\{H_{\nu}, \nu \in \Lambda\right\}, X$ be a relative generating set for $G$ with respect to $\left\{H_{\nu}, \nu \in \Lambda\right\}$ and $\mathcal{H}$ defined in (2.2). Let $H$ be a suitable subgroup of $G, t_{1}, \cdots, t_{m}$ arbitrary elements of $G$ and $N$ an arbitrary positive integer. Then there exists an epimorphism $\eta: G \rightarrow \bar{G}$ such that the following hold. 
(1) The group $\bar{G}$ is hyperbolic relative to $\left\{\eta\left(H_{\nu}\right), \nu \in \Lambda\right\}$.

(2) For any $i=1, \cdots, m$, we have $\eta\left(t_{i}\right) \in \eta(H)$.

(3) The restriction of $\eta$ to $\mathcal{H}$ is injective.

(4) The restriction of $\eta$ to the subset of elements of length at most $N$ with respect to $X \cup \mathcal{H}$ is injective.

(5) If all hyperbolic elements of $G$ have infinite order then all hyperbolic elements of $\bar{G}$ have infinite order.

Note that every free abelian group of finite rank is hyperbolic relative to itself. Hence, the following lemma holds.

Lemma 5.1.4. Every free abelian group of finite rank is fully residually- $\mathscr{R}$ in the sense of both Definition 5.1.1 and 5.1.2.

We now are able to prove the main result of this section.

Theorem 5.1.5. Let $G \in \mathscr{R}$. Every nontrivial finitely generated subgroup $H$ of $G$ is fully residually- $\mathscr{R}$ in the sense of Definition 5.1.1.

Proof. A torsion-free elementary group is necessarily cyclic and so belongs to $\mathscr{R}$. So, let $H$ be non-elementary. If $H$ is contained in a parabolic subgroup of $G$, then $H$ is a free abelian group of finite rank, hence it is fully residually- $\mathscr{R}$ in the sense of Definition 5.1.1 by Lemma 5.1.4. If $H$ contains a hyperbolic element then $H$ is suitable by Corollary 3.2.17. Since $G$ is finitely generated, Theorem 5.1.3 (2) implies that there exists a homomorphism $\eta: G \rightarrow \bar{G}$ such that $\eta(H)=\bar{G}$. Indeed, one can choose the elements $t_{1}, \ldots, t_{m}$ to be a generating set for $G$. Furthermore, Theorem 5.1.3 (4) yields that $\eta$ is injective on any finite set of elements in $G$. By Theorem 5.1.3 (1), (3) and (5), $\bar{G} \in \mathscr{R}$. Hence, by considering the homomorphism $\eta$ restricted to $H, H$ is fully residually- $\mathscr{R}$ in the sense of Definition 5.1.1.

Corollary 5.1.6. A finitely generated group is fully residually- $\mathscr{R}$ in the sense of Definition 5.1.1 if and only if it is fully residually- $\mathscr{R}$ in the sense of Definition 5.1.2.

Proof. Obviously, Definition 5.1.1 implies Definition 5.1.2. To prove the converse, let $L$ be a finitely generated group, and let $a_{1}, \ldots, a_{n}$ be nontrivial elements of $L$. If $L$ is fully residually- $\mathscr{R}$ in the sense of Definition 5.1 .2 then there is a group $G \in \mathscr{R}$ and a homomorphism $\phi: L \rightarrow G$ such that $\phi\left(a_{i}\right) \neq 1$, for all $i=1, \ldots, n$. We argue that there is an epimorphism $\alpha$ from $L$ onto a group in $\mathscr{R}$ such that the elements $a_{1}, \ldots, a_{n}$ survive in the image, as follows. Let $H=\phi(L) \subseteq G$. If $H$ is either free abelian, or elementary, or $H=G$ then $H \in \mathscr{R}$ and we are done. Otherwise, $H$ is a proper subgroup of $G$ and is suitable. Choose a finite generating set $t_{1}, \ldots, t_{m}$ for $G$ and an integer $N$ such that $N>\max \left\{\left|\phi\left(a_{i}\right)\right|_{G} \mid i=1, \ldots, n\right\}$, and let $\eta$ be a homomorphism from Theorem 5.1.5 such that $\eta(H)=\bar{G} \in \mathscr{R}$. Let $\mu=\left.\eta\right|_{H}$ be the restriction of $\eta$ to $H$. Then $\alpha=\mu \circ \phi: L \rightarrow \bar{G}$ is an epimorphism which has all of the required properties, by the proof of Theorem 5.1.5. Hence, $L$ is fully residually- $\mathscr{R}$ in the sense of Definition 5.1.1. 


\subsection{The generalization of Baumslag's theorem}

Recall that a group is called commutative transitive if for any nontrivial elements $g, h, f$, if $[g, h]=1$ and $[g, f]=1$ then $[h, f]=1$. Relatively hyperbolic groups are not commutative transitive in general. For instance, if $G$ is a non-abelian relatively hyperbolic group with torsion, then it may have a nontrivial finite centre (see Corollary 3.2.12). In this case, $G$ is not commutative transitive. However, toral relatively hyperbolic groups are commutative transitive as the following theorem shows.

Theorem 5.2.1. If $G$ is a toral relatively hyperbolic group, then $G$ is commutative transitive. In particular, if $G \in \mathscr{R}$ then $G$ is commutative transitive.

Proof. Suppose that there are nontrivial elements $x, y$ and $z \in G$ with $[x, y]=1$ and $[y, z]=1$. It follows from Corollary 3.2.5 that if one of $x, y, z$ is parabolic then so are the other two. Moreover, in this case all the three elements belong to one and the same parabolic subgroup. Since every parabolic subgroup is abelian, we have $[x, z]=1$. So, suppose that $x, y$ and $z$ are hyperbolic in $G$. Since $y x y^{-1}=x, y \in E(x)$ and so, $E(x) \subseteq E(y)$ by the first assertion of Theorem 3.2.6. Similarly, we have $E(y) \subseteq E(x)$. So, $E(x)=E(y)$. By a similar argument, we have $E(y)=E(z)$. Therefore, $x, y$ and $z$ are in $E(x)$. But Lemma 3.2 .9 implies that $E(x)$ is the maximal cyclic subgroup containing $x$; and hence, $[x, z]=1$. Thus, $G$ is commutative transitive.

Throughout the rest of this section, we use Definition 5.1 .2 of (fully) residually- $\mathscr{R}$ groups. We have shown in Section 5.1 that if $L$ is a finitely generated group then $L$ is (fully) residually- $\mathscr{R}$ in the sense of Definition 5.1.1 if and only if $L$ is (fully) residually- $\mathscr{R}$ in the sense of Definition 5.1.2,

Proposition 5.2.2. Suppose that $G$ is a fully residually- $\mathscr{R}$ group. Then $G$ is commutative transitive.

Proof. Let $x, y$ and $z$ be nontrivial elements in $G$; and let $[x, y]=1$ and $[y, z]=1$. We need to show that $[x, z]=1$. Suppose that $[x, z] \neq 1$. Since $G$ is fully residually $\mathscr{R}$, there is a homomorphism $\phi: G \rightarrow H \in \mathscr{R}$ such that $\phi(x), \phi(y), \phi(z)$ and $\phi([x, z])$ are nontrivial elements in $H$. But $\phi([x, y])$ and $\phi([y, z])$ are trivial in $H$. Since $H$ is commutative transitive by Theorem 5.2.1, we have $\phi([x, z])=1$. The contradiction shows that $[x, z]=1$.

Lemma 5.2.3. If $G$ is a residually- $\mathscr{R}$ group, then $G$ is torsion-free.

Proof. Assume that there exists a nontrivial torsion element $x \in G$. Since $G$ is residually$\mathscr{R}$, there is a homomorphism $\phi: G \rightarrow H \in \mathscr{R}$ such that $\phi(x) \neq 1$. Then $\phi(x)$ has infinite order in $H$, a contradiction. Therefore, $G$ is torsion-free.

Corollary 5.2.4. Suppose that a finitely generated abelian group $G$ is residually- $\mathscr{R}$. Then $G$ is fully residually- $\mathscr{R}$.

Proof. By Lemma 5.2.3, $G$ has no torsion elements hence, it is isomorphic to a free abelian group. Then by Lemma 5.1.4, $G$ is fully residually- $R$.

Lemma 5.2.5. Every abelian normal subgroup of a residually- $\mathscr{R}$ group is contained in its centre. 
Proof. Let $G$ be a residually- $\mathscr{R}$ group; let $A$ be an abelian normal subgroup of $G$; and let $Z(G)$ be the centre of $G$. Suppose that $A$ is not a subset of $Z(G)$. Then there exists a nontrivial element $a \in A \backslash Z(G)$ with $[a, g] \neq 1$ for some $g \in G$. Note that $[a, g] \in A$ as $A$ is normal in $G$. Since $G$ is residually- $\mathscr{R}$, there exists a homomorphism $\phi: G \rightarrow H \in \mathscr{R}$ such that $\phi([a, g]) \neq 1$; necessarily, $\phi(a) \neq 1$ and $\phi(g) \neq 1$. Then $\phi(G)$ is a non-abelian subgroup of $H$; in particular, $\phi(G)$ is a non-elementary non-parabolic subgroup of $H$. Note that $\phi(A)$ is abelian and normal in $\phi(G)$ as $A$ is abelian and normal in $G$. So, $\phi(A)$ is trivial by Corollary 3.2.12. It is impossible as $1 \neq \phi([a, g]) \in \phi(A)$. Therefore, we have $A \subseteq Z(G)$.

Example 5.2.6. The direct product $F_{2} \times \mathbb{Z}$ of a free group of rank 2 and an infinite cyclic group is residually- $\mathscr{R}$. Note that $F_{2} \times \mathbb{Z}$ is not commutative transitive since it is nonabelian with a nontrivial centre. By Proposition 5.2.2, $F_{2} \times \mathbb{Z}$ is not fully residually- $\mathscr{R}$.

Having proved that groups in $\mathscr{R}$ are commutative transitive, we can apply an argument due to B. Baumslag Bau67 to prove the following.

Proposition 5.2.7. Suppose that a finitely generated group $G$ is residually- $\mathscr{R}$ and commutative transitive. Then $G$ is fully residually- $\mathscr{R}$.

Proof. (B. Baumslag) Assume that the centre of $G$ is trivial; otherwise, $G$ would be abelian because it is commutative transitive and hence, $G$ is fully residually- $\mathscr{R}$ by Corollary 5.2 .4 .

Let $n$ be a positive integer and let $g_{1}, \ldots g_{n}$ be nontrivial elements of $G$. We claim that there exists a nontrivial $g \in G$ such that if $\phi(g) \neq 1$ for a homomorphism $\phi: G \rightarrow H \in \mathscr{R}$ then none of the elements $\phi\left(g_{1}\right), \cdots \phi\left(g_{n}\right)$ is trivial. We can prove this by induction on $n$. For $n=1$, it is true by taking $g=g_{1}$. Now, assume that there exists a nontrivial $g \in G$ such that the claim is true for nontrivial elements $g_{1}, \ldots, g_{k}$ where $k<n$. We need to show that there exists a nontrivial $g^{\prime} \in G$ such that the assertion holds for $g_{1}, \ldots, g_{k+1}$. Consider $c(x)=\left[g, x g_{k+1} x^{-1}\right]$ for $x \in G$. If $c(x)=1$ for all $x$, then $A=\left\langle x g_{k+1} x^{-1}\right|$ for every $\left.x \in G\right\rangle$ is abelian because $G$ is commutative transitive; in addition, $A$ is normal in $G$. Since the centre $Z(G)$ of $G$ is trivial, and by Lemma 5.2.5 $A \subset Z(G), A$ is trivial. But note that $g_{k+1} \in A$, which is impossible as $g_{k+1} \neq 1$. Hence, there exists $x$ in $G$ such that $c(x) \neq 1$. Choose $g^{\prime}=c(x)$. Note that $\phi\left(g^{\prime}\right) \neq 1$ implies that $\phi(g) \neq 1$ and $\phi\left(g_{k+1}\right) \neq 1$. So, none of the elements $\phi\left(g_{1}\right), \cdots, \phi\left(g_{k+1}\right)$ is trivial; and hence, the claim is proven.

Let $g$ be constructed as in the claim. Since $G$ is residually- $\mathscr{R}$, we have a homomorphism $\phi: G \rightarrow H \in \mathscr{R}$ such that $\phi(g) \neq 1$. We can conclude that $\phi\left(g_{i}\right) \neq 1$ for all $i=1, \cdots, n$; in particular, $G$ is fully residually- $\mathscr{R}$.

As a consequence of Propositions 5.2.2 and 5.2.7, we have the following generalization of B. Baumslag's theorem Bau67.

Theorem 5.2.8. A finitely generated group is fully residually- $\mathscr{R}$ if and only if it is residually- $\mathscr{R}$ and commutative transitive.

\subsection{Toral-limit groups}

In this section, we study the class of fully residually- $\mathscr{R}$ groups. We recall some definitions introduced by Miasnikov and Remeslennikov in the paper [MR96]. Let $G$ be a group. Recall that the centralizer of an element $u \in G$ in $G$ is denoted by $C_{G}(u)$. 
Definition 5.3.1. Let $G$ be a group. We say that a group $G_{1}$ is obtained from $G$ by an extension of a centralizer if, for some $u \in G, G_{1}$ is isomorphic to the free product of $G$ and $C_{G}(u) \times\langle t\rangle$ with amalgamation:

$$
G_{1} \cong G *_{C_{G}(u)}\left(C_{G}(u) \times\langle t\rangle\right) .
$$

Also, $G_{1}$ has a presentation as a HNN-extension, as follows:

$$
G_{1}=\left\langle G, t \mid\left[C_{G}(u), t\right]=1\right\rangle .
$$

A group obtained as the union of a chain of extensions of centralizers

$$
G=G_{0} \leq G_{1} \leq \cdots \leq G_{i} \leq G_{i+1} \leq \cdots \leq G_{k}
$$

is called an iterated extension of centralizers up to $k$, where

$$
G_{i+1}=\left\langle G_{i}, t_{i} \mid\left[C_{G_{i}}\left(u_{i}\right), t_{i}\right]=1\right\rangle, i=0,1, \ldots, k-1 .
$$

The following result is proved by Osin in Osi06b] stated as Theorem 3.4.4.

Theorem 5.3.2. Osi06b, Corollary 1.5] Suppose that $A$ and $B$ are groups, hyperbolic relative to $\left\{A_{\mu}\right\}_{\mu \in M} \cup\{K\}$ and $\left\{B_{\nu}\right\}_{\nu \in N}$, respectively. Assume in addition that $K$ is finitely generated and for some $\eta \in N$, there is a monomorphism $\xi: K \rightarrow B_{\eta}$. Then the amalgamated product $A *_{K=\xi(K)} B$ is hyperbolic relative to $\left\{A_{\mu}\right\}_{\mu \in M} \cup\left\{B_{\nu}\right\}_{\nu \in N}$.

Corollary 5.3.3. Let $G$ be a finitely generated group hyperbolic relative to a collection $\mathcal{H}=\left\{H_{\lambda}, \lambda \in \Lambda\right\}$ of subgroups; and let $\mathcal{P}=\mathcal{H} \backslash\left\{H_{\lambda}\right\}$ for some $\lambda \in \Lambda$. Suppose that $C$ is a subgroup of $H_{\lambda}$, and let $K=\left\langle H_{\lambda}, t \mid[C, t]=1\right\rangle$. Then $G *_{C}(C \times\langle t\rangle)$ is hyperbolic relative to $\mathcal{P} \cup\{K\}$.

Proof. Since $G$ is finitely generated, each subgroup in $\mathcal{H}$ is finitely generated, by Theorem 3.1.1. Note that $K$ is hyperbolic relative to itself and that $H_{\lambda}$ embeds into $K$. Hence, the amalgamated free product $G_{1}=G *_{H_{\lambda}} K$ is hyperbolic relative to $\mathcal{P} \cup\{K\}$, by Theorem 5.3.2. Clearly, $G_{1}$ is isomorphic to the amalgamated product $G *_{C}(C \times\langle t\rangle)$. Therefore, $G *_{C}(C \times\langle t\rangle)$ is hyperbolic relative to $\mathcal{P} \cup\{K\}$.

Proposition 5.3.4. Let $\mathcal{C}$ be the class of finitely generated torsion-free relatively hyperbolic groups, and let $G \in \mathcal{C}$. Suppose that $G_{k}$ is obtained from $G$ by an iterated extension of centralizers up to $k$. Then $G_{k} \in \mathcal{C}$, for any $k \geq 1$. In fact, if $G \in \mathscr{R}$ then $G_{k} \in \mathscr{R}$, for any $k \geq 1$.

Proof. Let $G \in \mathcal{C}$ be hyperbolic relative to a collection $\mathcal{H}=\left\{H_{\lambda}, \lambda \in \Lambda\right\}$ of subgroups, and let $u$ be a hyperbolic element in $G$. As $G$ is torsion-free, by Lemma 3.2.9, $E(u)=\langle a\rangle$ for some $a \in G$, and so $C_{G}(u)=\langle a\rangle$. By the second assertion of Theorem 3.2.6, $G$ is hyperbolic relative to $\mathcal{H} \cup\{\langle a\rangle\}$. Corollary 5.3.3 implies that

$$
G_{1}=\langle G, t \mid[a, t]=1\rangle=G *_{\langle a\rangle}(\langle a\rangle \times\langle t\rangle)
$$

is hyperbolic relative to $\mathcal{H} \cup(\langle a\rangle \times\langle t\rangle)$. As an amalgamated product of finitely generated torsion-free groups, $G_{1}$ is finitely generated and torsion-free. It follows that $G_{1} \in \mathcal{C}$. Note that if $G \in \mathscr{R}$ then the subgroups in $\mathcal{H}$ and $\langle a\rangle \times\langle t\rangle$ are all finitely generated free abelian and so $G_{1} \in \mathscr{R}$. 
Now, suppose that $u$ is a parabolic element in $G$, then $u^{\prime}=g^{-1} u g \in H_{\lambda}$ for some $H_{\lambda} \in \mathcal{H}$ and $g \in G$. Let $\mathcal{P}=\mathcal{H} \backslash\left\{H_{\lambda}\right\}$. Denote $C=C_{G}\left(u^{\prime}\right)$; by Corollary 3.2.5, $C \subseteq H_{\lambda}$. Set $K=\left\langle H_{\lambda}, t ;[C, t]=1\right\rangle$, then $K$ is finitely generated and torsion free. It follows that $G^{\prime}=G *_{H_{\lambda}} K$ is finitely generated and torsion-free; and so is $G_{1}=G *_{C}(C \times\langle t\rangle)$ as $G_{1} \cong G^{\prime}$. By Corollary 5.3.3, $G_{1}$ is hyperbolic relative to $\mathcal{P} \cup\{K\}$. Thus, $G_{1} \in \mathcal{C}$. Note that if $G \in \mathscr{R}$ then $C=H_{\lambda}$ and so $K$ is abelian; therefore, $G_{1} \in \mathscr{R}$.

An easy induction on $k$ shows that $G_{k} \in \mathcal{C}$. If $G \in \mathscr{R}$ then either $C_{G_{i}}\left(u_{i}\right)$ is a maximal cyclic hyperbolic subgroup, or $C_{G_{i}}\left(u_{i}^{\prime}\right)$ is an abelian parabolic subgroup, for each $i$. By induction, we conclude that $G_{k} \in \mathscr{R}$, for all $k$.

Observe that every nontrivial parabolic subgroup of $G \in \mathscr{R}$ is proper if and only if $G$ is non-abelian. We denote the class of non-abelian groups in $\mathscr{R}$ by $\mathscr{G}$. The statement of Theorem C from [KM12] is equivalent to the following; for instance, see [BMR99, [MR96].

Theorem 5.3.5. [KM12, Theorem C] Suppose that $\Gamma \in \mathscr{G}$. Then a finitely generated group $G$ is fully residually- $\Gamma$ if and only if $G$ embeds into a group $\bar{\Gamma}$ obtained from $\Gamma$ by an iterated extension of centralizers up to a finite number $k$.

Theorem 5.3.6. Let $\Gamma \in \mathscr{R}$. If a finitely generated group $G$ is fully residually- $\Gamma$ then $G$ embeds into a group $\bar{\Gamma} \in \mathscr{R}$.

Proof. Note that $\mathscr{R}=\mathscr{G} \cup\{$ all abelian groups in $\mathscr{R}\}$. If $\Gamma \in \mathscr{G}$, then the statement immediately follows from Proposition 5.3.4 and Theorem 5.3.5. If $\Gamma \in \mathscr{R}$ is a finitely generated free abelian group then $G$ is free abelian and so $G \in \mathscr{R}$.

\subsection{The Baumslag-Solitar groups}

The statement of Theorem 5.3.6 can be contrasted with the case when one considers torsion-free fully residually- $\mathscr{H}$ groups, where $\mathscr{H}$ is the class of hyperbolic groups, see Proposition 5.4 .4 below.

Recall that the presentation $B S(p, q)=\left\langle a, b \mid b a^{p} b^{-1}=a^{q}\right\rangle$ with $p, q \in \mathbb{Z}$ defines a Baumslag-Solitar group. Whereas the integers $p$ and $q$ can be arbitrary, we always assume that $p$ and $q$ are nonzero.

Lemma 5.4.1. Osi06c, Corollary 4.22] Let $G$ be a finitely generated group hyperbolic relative to a collection of subgroups $\left\{H_{1}, \cdots, H_{m}\right\}$. Suppose that

$$
B \cong B S(p, q)=\left\langle a, b \mid b a^{p} b^{-1}=a^{q}\right\rangle
$$

with $p q \neq 0$ is a subgroup of $G$. Then $B$ is a parabolic subgroup of $G$.

Proof. The assumption $p q \neq 0$ implies that $B$ is torsion-free.

Firstly, assume that $a$ is parabolic. Then $a$ is contained in a parabolic subgroup $P$, and we have that $a^{q} \in P \cap b \mathrm{~Pb}^{-1}$ contradicting to Corollary 3.2.4, unless $b \in P$. So, $B$ is a parabolic subgroup.

Now, assume that $a$ is hyperbolic. Then $a \in E(a)$ by the first assertion of Theorem 3.2.6, and $E(a)$ is hyperbolic as an abstract group. By Lemma 3.2.13, $q= \pm p$ and so, by the first assertion of Theorem 3.2.6, $b \in E(a)$. Therefore, $B \cong\left\langle a, b \mid b a^{p} b^{-1}=a^{ \pm p}\right\rangle$ is a subgroup of $E(a)$. However, the subgroup $\left\langle a^{p}, b^{2}\right\rangle$ of $B$ is isomorphic to a free abelian group of rank 2 , which is a contradiction. Therefore, $a$ cannot be a hyperbolic element of $G$. 
Corollary 5.4.2. Let $B \cong B S(1, q)$, where $q \geq 2$. Then $B$ is not a subgroup of a group in $\mathscr{R}$.

Proof. Let $G$ be a group in $\mathscr{R}$. Assume that $B$ is a subgroup of $G$. Then $B$ is a parabolic subgroup by Lemma 5.4.1 hence, $B$ is abelian, which is a contradiction.

The following Theorem is due to Meskin [Mes72].

Theorem 5.4.3. Mes72, Theorem $C] B S(p, q)$ is residually finite if and only if $|p|=1$ or $|q|=1$ or $|p|=|q|$.

Proposition 5.4.4. Let $\mathscr{H}$ denote the class of hyperbolic groups. Let $B=B S(1, q)$ where $q \geq 2$. Then $B$ is torsion-free fully residually- $\mathscr{H}$. In particular, torsion-free fully residually- $\mathscr{H}$ groups do not embed into groups in $\mathscr{R}$.

Proof. Note that $B$ is torsion-free. By Theorem 5.4.3, $B$ is residually finite. Hence, $B$ is fully residually finite. It follows that $B$ is fully residually- $\mathscr{H}$, as the class $\mathscr{H}$ contains all finite groups. Note that $B$ cannot be embedded into a group from $\mathscr{R}$ by Corollary 5.4.2. Therefore, the result follows. 


\section{Chapter 6}

\section{Future directions}

\subsection{The conjugacy problem for relatively hyperbolic groups}

In the future, I would like to generalize the following conjugacy problems in hyperbolic groups to relatively hyperbolic groups.

The first one is proved by Bridson and Howie in [BH05]. Let $G$ be a group; and let $A=\left[a_{1}, \cdots, a_{m}\right]$ and $B=\left[b_{1}, \cdots, b_{m}\right]$ be two finite sets of elements in $G$. We say that $A$ and $B$ are conjugate in $G$ if there exists $x \in G$ such that $x^{-1} a_{i} x=b_{i}$ for all $i=1, \cdots, m$. Then the conjugacy (search) problem for two elements in $G$ can be generalized to for two finite subsets in $G$. Bridson and Howie provided a quadratic time algorithm that solves the conjugacy search problem between finite subsets in any torsion-free hyperbolic group.

The second one is proved by Epstein and Holt in [EH06]. In Theorem 1.1.5, stated in the introduction, the time complexity of the algorithm solving the conjugacy problem for relatively hyperbolic groups is not as "efficient" as the one provided by Epstein and Holt in [EH06], which runs a linear time for hyperbolic groups. In what follows, when we speak of a word $w$ as a product of generators in a group, we mean that $w$ is also a group element. The key idea of Epstein and Holt's linear time algorithm A is that transforms a word in a hyperbolic group to a unique reduced conjugate all of whose cyclic permutations are geodesics. Let $u$ and $v$ be such words after applied the algorithm A. If $u$ and $v$ are conjugate, then $u$ is conjugate to a cyclic permutation $v^{\prime}$ of $v$ with the shortest length of a conjugating element $z$ bounded by a constant. For each $z$, one applies the algorithm A on the word $z u z^{-1}$. If $u$ and $v$ are conjugate, then $z u z^{-1}$ is a subword of $v^{2}$ because of the uniqueness. By the Knuth-Morris-Pratt string searching algorithm, described in [KMP77], one can be verified in linear time.

\subsection{Embedding to $\mathscr{R}$}

Let $\Gamma \in \mathscr{R}$ be non-abelian and non-elementary. Kharlampovich and Miasnikov proved in [KM12, also stated in Theorem 5.3.5 that a finitely generated fully residually- $\Gamma$ group $G$ embeds into an iterated extension of centralizers of $\Gamma$. In Theorem 5.3.6, we deduce from their theorem that every finitely generated fully residually- $\Gamma$ group embeds into a group from $\mathscr{R}$. On the other hand, we can ask the following question.

Question 6.2.1. Let $G$ be a finitely generated fully residually- $R$ group. Does $G$ embed into a group in $\mathscr{R}$ ? 
Let $\mathscr{H}$ be the class of hyperbolic groups. Proposition 5.4 .4 gives an example of a finitely generated torsion-free fully residually- $\mathscr{H}$ group, i.e., a Baumslag-Solitar group $B_{q}=B S(1, q)$ for $q \geq 2$ that does not embed into a group from $\mathscr{R}$. Note that, whereas the groups $B_{q}=B S(1, q), q \geq 2$, are torsion-free, in our proof the hyperbolic quotients of $B_{q}$ all have torsion elements. Since every group in $\mathscr{R}$ is torsion-free, Baumslag-Solitar groups $B_{q}=B S(1, q)$ for $q \geq 2$ cannot be a counter-example to the above question. I would like to obtain some results from the above question. 


\section{Bibliography}

$\left[\mathrm{ABC}^{+} 91\right]$ Juan M Alonso, Thomas Brady, Daryl Cooper, Vincent Ferlini, Martin Lustig, Michael Mihalik, Michael Shapiro, and Hamish Short. Notes on word hyperbolic groups. In Group theory from a geometrical viewpoint. Singapore: World Scientific, 1991.

[Ali05] Emina Alibegović. A combination theorem for relatively hyperbolic groups. Bulletin of the London Mathematical Society, 37(3):459-466, 2005.

[AMO07] G Arzhantseva, A Minasyan, and D Osin. The SQ-universality and residual properties of relatively hyperbolic groups. Journal of Algebra, 315(1):165177, 2007.

[Bau67] Benjamin Baumslag. Residually free groups. Proceedings of the London Mathematical Society, 3(3):402-418, 1967.

[BH99] Martin R Bridson and André Haefliger. Metric spaces of non-positive curvature, volume 319. Springer Science \& Business Media, 1999.

[BH05] Martin R Bridson and James Howie. Conjugacy of finite subsets in hyperbolic groups. International Journal of Algebra and Computation, 15(04):725-756, 2005.

[BMR99] Gilbert Baumslag, Alexei Myasnikov, and Vladimir Remeslennikov. Algebraic geometry over groups I. Algebraic sets and ideal theory. Journal of Algebra, 219(1):16-79, 1999.

[Boo59] William W Boone. The word problem. Annals of mathematics, pages 207265, 1959 .

[Bow12] Brian H Bowditch. Relatively hyperbolic groups. International Journal of Algebra and Computation, 22(03):1250016, 2012.

[Bum04] Inna Bumagin. The conjugacy problem for relatively hyperbolic groups. Algebraic 83 Geometric Topology, 4(2):1013-1040, 2004.

[Bum15] Inna Bumagin. Time complexity of the conjugacy problem in relatively hyperbolic groups. International Journal of Algebra and Computation, 25(05):689-723, 2015.

[BZ16] Inna Bumagin and Ming Ming Zhang. On fully residually- $\mathscr{R}$ groups. Communications in Algebra, 44(7):2813-2827, 2016. 
[CDP89] Michel Coornaert, Thomas Delzant, and Athanase Papadopoulos. Notes sur les groupes hyperboliques de gromov: chapitres 1 à 12. Technical report, Strasbourg 1, 1989.

[CFR16] Laura Ciobanu, Ben Fine, and Gerhard Rosenberger. Classes of groups generalizing a theorem of Benjamin Baumslag. Communications in Algebra, 44(2):656-667, 2016.

[Dah03] François Dahmani. Combination of convergence groups. Geometry 85 Topology, 7(2):933-963, 2003.

[Dah08] François Dahmani. Finding relative hyperbolic structures. Bulletin of the London Mathematical Society, 40(3):395-404, 2008.

[Deh11] Max Dehn. Über unendliche diskontinuierliche gruppen. Mathematische Annalen, 71(1):116-144, 1911.

[Deh12] Max Dehn. Transformation der kurven auf zweiseitigen flächen. Mathematische Annalen, 72(3):413-421, 1912.

[DG13] François Dahmani and Vincent Guirardel. Presenting parabolic subgroups. Algebraic \& Geometric Topology, 13(6):3203-3222, 2013.

[DGO17] François Dahmani, Vincent Guirardel, and Denis Osin. Hyperbolically embedded subgroups and rotating families in groups acting on hyperbolic spaces, volume 245. American Mathematical Society, 2017.

[EH06] David Epstein and Derek Holt. The linearity of the conjugacy problem in word-hyperbolic groups. International Journal of Algebra and Computation, 16(02):287-305, 2006.

[Far98] Benson Farb. Relatively hyperbolic groups. Geometric and functional analysis, 8(5):810-840, 1998.

[Fri60] A. A. Fridman. On the relation between the word problem and the conjugacy problem in finitely defined groups. Trudy Moskov. Mat. Obshch., 9:329-365, 1960.

[GdLH] E Ghys and P de La Harpe. editeurs, sur les groupes hyperboliques d'apr es mikhael gromov. Prog. in Math, 83.

[Gro87] Mikhael Gromov. Hyperbolic groups. Essays in group theory, 8(75-263):2, 1987.

[Gro05] Daniel Groves. Limit groups for relatively hyperbolic groups, II: Makaninrazborov diagrams. Geometry \&6 Topology, 9(4):2319-2358, 2005.

[GS91a] SM Gersten and HB Short. Rational subgroups of biautomatic groups. Annals of mathematics, pages 125-158, 1991.

[GS91b] Stephen M Gersten and Hamish Short. Small cancellation theory and automatic groups: Part II. Inventiones mathematicae, 105(1):641-662, 1991. 
[GW16] Daniel Groves and Henry Wilton. The structure of limit groups over hyperbolic groups. arXiv preprint arXiv:1603.07187, 2016.

[Hou07] Abderezak Houcine. Limit groups of equationally noetherian groups. Geometric group theory, pages 103-119, 2007.

[Hru10] G Christopher Hruska. Relative hyperbolicity and relative quasiconvexity for countable groups. Algebraic \& Geometric Topology, 10(3):1807-1856, 2010.

[JOR10] R. Ji, C. Ogle, and B. Ramsey. Relatively hyperbolic groups, rapid decay algebras, and a generalization of the bass conjecture. Journal of Noncommuative Geometry, 4(1):83-124, 2010.

[KM96] O Kharlampovich and A Myasnikov. Description of fully residually free groups and irreducible affine varieties over a free group. Summer School in Group Theory in Banff, 17:71-80, 1996.

[KM98a] Olga Kharlampovich and Alexei Myasnikov. Irreducible affine varieties over a free group: I. irreducibility of quadratic equations and nullstellensatz. Journal of Algebra, 200(2):472-516, 1998.

[KM98b] Olga Kharlampovich and Alexei Myasnikov. Irreducible affine varieties over a free group: II. systems in triangular quasi-quadratic form and description of residually free groups. Journal of Algebra, 200(2):517-570, 1998.

[KM06] Olga Kharlampovich and Alexei Myasnikov. Elementary theory of free nonabelian groups. Journal of Algebra, 302(2):451-552, 2006.

[KM12] O. Kharlampovich and A. Myasnikov. Limits of relatively hyperbolic groups and Lyndon's completions. Journal of the European Math Society, 14:659680, 2012.

[KM13] Olga Kharlampovich and Alexei Myasnikov. Decidability of the elementary theory of a torsion-free hyperbolic group. arXiv preprint arXiv:1303.0760, 2013.

[KMP77] Donald E Knuth, James H Morris, Jr, and Vaughan R Pratt. Fast pattern matching in strings. SIAM journal on computing, 6(2):323-350, 1977.

[Lys90] Igor Geront'evich Lysenok. On some algorithmic properties of hyperbolic groups. Mathematics of the USSR-Izvestiya, 35(1):145, 1990.

[Mal40] Anatolii Malcev. On isomorphic matrix representations of infinite groups. Matematicheskii Sbornik, 50(3):405-422, 1940.

[Mes72] Stephen Meskin. Nonresidually finite one-relator groups. Transactions of the American Mathematical Society, 164:105-114, 1972.

[MM00] Howard A Masur and Yair N Minsky. Geometry of the complex of curves II: Hierarchical structure. Geometric and Functional Analysis, 10(4):902-974, 2000. 
[MMNV15] Jeremy Macdonald, Alexei Miasnikov, Andrey Nikolaev, and Svetla Vassileva. Logspace and compressed-word computations in nilpotent groups. Preprint, 2015.

[MP09] Eduardo Martínez-Pedroza. Combination of quasiconvex subgroups of relatively hyperbolic groups. Groups, Geometry, and Dynamics, 3(2):317-342, 2009.

[MR96] Alexei G Myasnikov and Vladimir N Remeslennikov. Exponential groups II: Extensions of centralizers and tensor completion of csa-groups. International Journal of Algebra and Computation, 6(06):687-711, 1996.

[MRUV10] Alexei Myasnikov, Vitalii Roman'kov, Alexander Ushakov, and Anatoly Vershik. The word and geodesic problems in free solvable groups. Transactions of the American Mathematical Society, 362(9):4655-4682, 2010.

[MSU11] Alexei G Myasnikov, Vladimir Shpilrain, and Alexander Ushakov. Noncommutative Cryptography and Complexity of Group-theoretic Problems. Number 177. American Mathematical Soc., 2011.

[Nov54] Petr Sergeevich Novikov. Unsolvability of the conjugacy problem in group theory. Izv. Akad. Nauk SSSR, Ser. Mat., 18:485-524, 1954.

[Nov55] Petr Sergeevich Novikov. On the algorithmic unsolvability of the word problem in group theory. Trudy Matematicheskogo Instituta imeni VA Steklova, $44: 3-143,1955$.

[O'C12] Zoe O'Connor. Conjugacy search problem for relatively hyperbolic groups. arXiv preprint arXiv:1211.5561, 2012.

[Ol'92] A Yu Ol'shanskii. Almost every group is hyperbolic. International Journal of Algebra and Computation, 2(01):1-17, 1992.

[Ol'93] A Yu Ol'shanskii. On residualing homomorphisms and G-subgroups of hyperbolic groups. International Journal of Algebra and Computation, 3(04):365409, 1993.

[Osi06a] Denis V Osin. Elementary subgroups of relatively hyperbolic groups and bounded generation. International Journal of Algebra and Computation, 16(01):99-118, 2006.

[Osi06b] Denis V Osin. Relative dehn functions of amalgamated products and HNNextensions. Contemporary Mathematics, 394:209, 2006.

[Osi06c] Denis V Osin. Relatively hyperbolic groups: intrinsic geometry, algebraic properties, and algorithmic problems, volume 843. American Mathematical Soc., 2006.

[Osi10] Denis Osin. Small cancellations over relatively hyperbolic groups and embedding theorems. Annals of mathematics, pages 1-39, 2010.

[Osi16] Denis Osin. Acylindrically hyperbolic groups. Transactions of the American Mathematical Society, 368(2):851-888, 2016. 
[RW10] Cornelius Reinfeldt and Richard Weidmann. Makanin-Razborov diagrams for hyperbolic groups. preprint, 2010.

[Sel01] Zlil Sela. Diophantine geometry over groups I: Makanin-Razborov diagrams. Publications Mathematiques de l'IHES, 93(1):31-106, 2001.

[Sel06] Zlil Sela. Diophantine geometry over groups VI: The elementary theory of a free group. Geometric and Functional Analysis, 16(3):707-730, 2006.

[Sel09] Zlil Sela. Diophantine geometry over groups VII: The elementary theory of a hyperbolic group. Proceedings of the London Mathematical Society, 99(1):217-273, 2009.

[Sta68] John R Stallings. On torsion-free groups with infinitely many ends. Annals of Mathematics, pages 312-334, 1968.

[Vas11] Svetla Vassileva. Polynomial time conjugacy in wreath products and free solvable groups. Groups-Complexity-Cryptology, 3(1):105-120, 2011. 